\title{
Radical Beckmann Rearrangement and Its Application in the Formal Total Synthesis of Antimalarial Natural Product Isocryptolepine via C-H Activation
}

\author{
Pankaj S. Mahajan, Vivek T. Humne, Subhash D. Tanpure, and Santosh B. Mhaske* \\ CSIR-National Chemical Laboratory, Division of Organic Chemistry, Pune 411 008, India \\ Email: sb.mhaske@ncl.res.in
}

Table of Contents:

Pages

1. General information.............................................

2. General experimental procedure for radical Beckmann.................2

3. Characterization data of Beckmann products...........................3-11

4. Experimental procedure and data for Scheme 5....................12-18

5. Radical trapping experiments.................................... 19

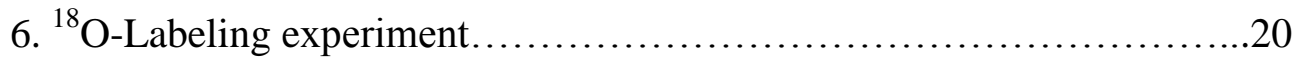

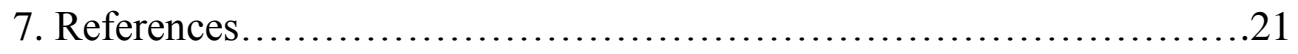

8. Copies of ${ }^{1} \mathrm{H},{ }^{13} \mathrm{C}$, DEPT, GC/LCMS and HRMS spectra...................22-107 


\section{General information:}

All reagents and solvents were used as received from commercial sources. All experiments were carried out under argon atmosphere unless otherwise noted. Ketoximes/aldoximes were prepared as per the known procedure. ${ }^{18} \mathrm{O}$-enriched $\mathrm{NaN}^{18} \mathrm{O}_{2}$ was prepared according to the literature procedure. $^{2}$ Pre-coated plates (silica gel $60 \mathrm{PF} 254,0.25 \mathrm{~mm}$ or $0.5 \mathrm{~mm}$ ) were utilized for thin layer chromatography (TLC). Column chromatographic purifications were carried out on flash silica-gel (240-400 mesh) using petroleum ether and ethyl acetate as eluents. The ${ }^{1} \mathrm{H},{ }^{13} \mathrm{C}$ NMR spectra were recorded on 200/400/500 MHz, and 50/100/125 MHz NMR spectrometers, respectively in $\mathrm{CDCl}_{3} / \mathrm{DMSO}-d_{6}$. Chemical shifts were reported as $\delta$ values from standard peaks. Melting points were recorded are uncorrected. Mass spectra were taken on LC-MS (ESI) or GCMS spectrometer. HRMS were scanned at NCL, Pune. 


\section{General experimental procedure for the radical Beckmann rearrangement:}

All the reactions were performed on $50 \mathrm{mg}$ of ketoxime/aldoxime.

The solution of ketoxime (1 equiv), ammonium persulfate (1.5 equiv) and DMSO (6 equiv) in 1,4-dioxane $(2 \mathrm{~mL})$ was heated at $100{ }^{\circ} \mathrm{C}$ in a Schlenk tube under argon atmosphere until completion of the reaction as indicated by thin layer chromatography. After completion, the reaction mixture was filtered through a cotton plug and 1,4-dioxane was evaporated under vacuum. The residue was dissolved in ethyl acetate $(10 \mathrm{~mL})$ and washed with warm water $(4$ $\mathrm{mL})$ and brine $(3 \mathrm{~mL} \times 2)$. The organic layer was dried over anhydrous sodium sulfate and the crude product was purified by flash column chromatography using a gradient of ethyl acetate and petroleum ether to furnish corresponding amides in good to excellent yields. 


\section{Characterization Data of Beckmann rearrangement products:}

\section{$N$-phenylacetamide: ${ }^{3}$}

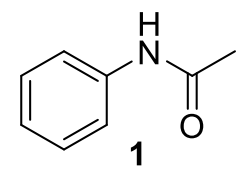

Reaction time: $3 \mathrm{~h} ; \mathrm{R}_{f}=0.3$ (EtOAc:Pet. Ether, 2:3). White solid; $46.6 \mathrm{mg}, 93 \%, \mathrm{mp} 114-115{ }^{\circ} \mathrm{C}$;

${ }^{1} \mathrm{H}$ NMR (400 MHz, $\left.\mathrm{CDCl}_{3}\right): \delta 7.87(\mathrm{bs}, 1 \mathrm{H}), 7.51(\mathrm{~d}, J=7.8 \mathrm{~Hz}, 2 \mathrm{H}), 7.30(\mathrm{t}, J=7.3 \mathrm{~Hz}, 2 \mathrm{H})$, $7.10(\mathrm{t}, J=7.3 \mathrm{~Hz}, 1 \mathrm{H}), 2.17(\mathrm{~s}, 3 \mathrm{H}) ;{ }^{13} \mathrm{C} \mathrm{NMR}\left(100 \mathrm{MHz}, \mathrm{CDCl}_{3}\right): \delta 168.8,137.8,128.9,124.3$, 120.0, 24.4; GC-MS $\left(\mathrm{M}^{+}\right) 135$.

\section{$N$-(p-tolyl)acetamide: ${ }^{3}$}

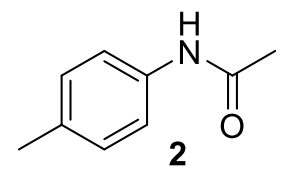

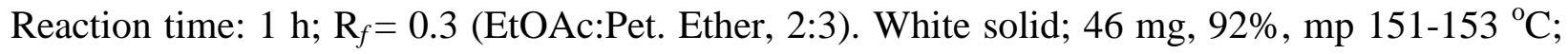

${ }^{1} \mathrm{H}$ NMR (400 MHz, $\left.\mathrm{CDCl}_{3}\right): \delta 7.38(\mathrm{bs}, 1 \mathrm{H}), 7.38(\mathrm{~d}, J=8.1 \mathrm{~Hz}, 2 \mathrm{H}), 7.12(\mathrm{~d}, J=8.1 \mathrm{~Hz}, 2 \mathrm{H})$, $2.31(\mathrm{~s}, 3 \mathrm{H}), 2.16(\mathrm{~s}, 3 \mathrm{H}) ;{ }^{13} \mathrm{C} \mathrm{NMR}\left(100 \mathrm{MHz}, \mathrm{CDCl}_{3}\right): \delta 168.3,135.3,133.9,129.4,120.0$, 24.5, 20.8; GC-MS $\left(\mathrm{M}^{+}\right) 149$. 


\section{$N$-(4-isobutylphenyl)acetamide:}

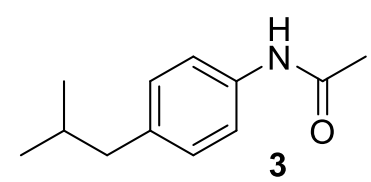

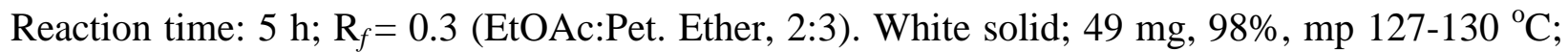

${ }^{1} \mathrm{H}$ NMR (400 MHz, $\left.\mathrm{CDCl}_{3}\right): \delta 7.41$ (bs, $\left.1 \mathrm{H}\right), 7.40(\mathrm{~d}, J=8.3 \mathrm{~Hz}, 2 \mathrm{H}), 7.09(\mathrm{~d}, J=8.3 \mathrm{~Hz}, 2 \mathrm{H})$, $2.43(\mathrm{~d}, J=7.3 \mathrm{~Hz}, 2 \mathrm{H}), 2.16(\mathrm{~s}, 3 \mathrm{H}), 1.83(\mathrm{~m}, 1 \mathrm{H}), 0.89(\mathrm{~d}, J=8 \mathrm{~Hz}, 6 \mathrm{H}) ;{ }^{13} \mathrm{C} \mathrm{NMR}(100 \mathrm{MHz}$ $\left.\mathrm{CDCl}_{3}\right): \delta 168.3,137.8,135.5,129.5,119.8,44.8,30.2,24.5,22.3 ;$ HRMS-ESI $(\mathrm{m} / z)$ calcd $\left[\mathrm{C}_{12} \mathrm{H}_{17} \mathrm{ON}+\mathrm{H}\right]^{+}:$192.1383, found 192.1380.

\section{$N$-(4-fluorophenyl)acetamide: ${ }^{4}$}

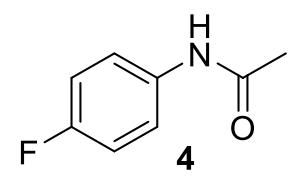

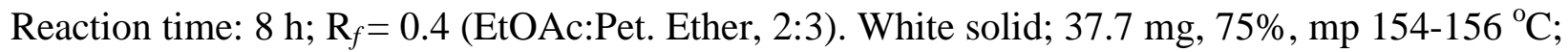

${ }^{1} \mathrm{H}$ NMR (400 MHz, $\left.\mathrm{CDCl}_{3}\right): \delta 7.47(\mathrm{bs}, 1 \mathrm{H}), 7.45\left(\mathrm{dd}, J_{1}=4.9 \mathrm{~Hz}, J_{2}=9.0 \mathrm{~Hz}, 2 \mathrm{H}\right), 7.00(\mathrm{t}, J$ $=8.6 \mathrm{~Hz}, 2 \mathrm{H}), 2.16(\mathrm{~s}, 3 \mathrm{H}) ;{ }^{13} \mathrm{C} \mathrm{NMR}\left(100 \mathrm{MHz}, \mathrm{CDCl}_{3}\right): \delta 168.4,160.6$ and $158.1(\mathrm{~d}, J=243.5$ $\mathrm{Hz}, 1 \mathrm{C}), 133.8,121.83$ and $121.76(\mathrm{~d}, J=7.7 \mathrm{~Hz}, 1 \mathrm{C}), 115.7$ and $115.5(\mathrm{~d}, J=22.4 \mathrm{~Hz}, 1 \mathrm{C})$, 24.3; GC-MS $\left(\mathrm{M}^{+}\right) 153$. 


\section{$N$-(4-chlorophenyl)acetamide: ${ }^{4}$}

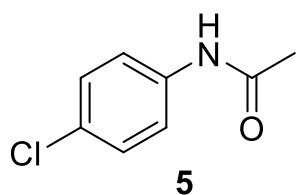

Reaction time: $8 \mathrm{~h} ; \mathrm{R}_{f}=0.4$ (EtOAc:Pet. Ether, 2:3). White solid; $37.2 \mathrm{mg}$, 74\%, $\mathrm{mp} \mathrm{177-180}{ }^{\circ} \mathrm{C}$; ${ }^{1} \mathrm{H} \mathrm{NMR}\left(500 \mathrm{MHz}, \mathrm{CDCl}_{3}\right): \delta 7.46(\mathrm{~d}, J=8.2 \mathrm{~Hz}, 2 \mathrm{H}), 7.29-7.27(\mathrm{~m}, 3 \mathrm{H}), 2.18(\mathrm{~s}, 3 \mathrm{H}) ;{ }^{13} \mathrm{C}$ NMR (125 MHz, $\left.\mathrm{CDCl}_{3}\right): \delta 168.3,136.4,129.3,129.0,121.1,24.6$; GC-MS $\left(\mathrm{M}^{+}\right) 169$.

\section{$N$-(4-bromophenyl)acetamide: ${ }^{5}$}

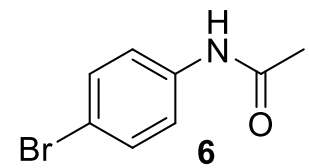

Reaction time: $8 \mathrm{~h} ; \mathrm{R}_{f}=0.4$ (EtOAc:Pet. Ether, 2:3). White solid; $41.1 \mathrm{mg}, 82 \%, \mathrm{mp} 165-168{ }^{\circ} \mathrm{C}$;

${ }^{1} \mathrm{H}$ NMR (400 MHz, $\left.\mathrm{CDCl}_{3}\right): \delta 7.42$ (s, 4H), 7.35 (bs, 1H), 2.18 (s, 3H); ${ }^{13} \mathrm{C} \mathrm{NMR}(100 \mathrm{MHz}$, $\left.\mathrm{CDCl}_{3}\right): \delta 168.3,136.9,131.9,121.3,116.8,24.6$; GC-MS $\left(\mathrm{M}^{+}\right) 213$. 


\section{$N$-(3-fluoro-4-methoxyphenyl)acetamide:}

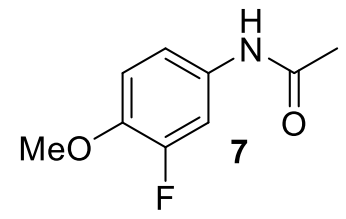

Reaction time: $4 \mathrm{~h} ; \mathrm{R}_{f}=0.5$ (EtOAc:Pet. Ether, 1:1). White solid; $39 \mathrm{mg}, 78 \%, \mathrm{mp} 165-168{ }^{\circ} \mathrm{C}$; ${ }^{1} \mathrm{H}$ NMR (400 MHz, $\left.\mathrm{CDCl}_{3}\right): \delta 7.42(\mathrm{bs}, 1 \mathrm{H}), 7.41(\mathrm{dd}, J=12.7,2.2 \mathrm{~Hz}, 1 \mathrm{H}), 7.12(\mathrm{~d}, J=8.8$ $\mathrm{Hz}, 1 \mathrm{H}), 6.89(\mathrm{t}, \quad J=9.0 \mathrm{~Hz}, 1 \mathrm{H}), 3.86(\mathrm{~s}, 3 \mathrm{H}), 2.15(\mathrm{~s}, 3 \mathrm{H}) ;{ }^{13} \mathrm{C}$ NMR $\left(100 \mathrm{MHz}, \mathrm{CDCl}_{3}\right): \delta$ 168.3, 153.2 and $150.8(\mathrm{~d}, J=245.1 \mathrm{~Hz}, 1 \mathrm{C}), 144.4$ and $144.3(\mathrm{~d}, J=10.8 \mathrm{~Hz}, 1 \mathrm{C}), 131.3$ and $131.2(\mathrm{~d}, J=9.3 \mathrm{~Hz}, 1 \mathrm{C}), 115.72$ and $115.69(\mathrm{~d}, J=3.1 \mathrm{~Hz}, 1 \mathrm{C}), 113.62$ and $113.60(\mathrm{~d}, J=2.3$ $\mathrm{Hz}, 1 \mathrm{C}), 109.4$ and $109.2(\mathrm{~d}, J=23.1 \mathrm{~Hz}, 1 \mathrm{C}), 56.6,24.3 ;$ HRMS-ESI $(\mathrm{m} / \mathrm{z})$ calcd $\left[\mathrm{C}_{9} \mathrm{H}_{10} \mathrm{O}_{2} \mathrm{NF}+\mathrm{H}\right]^{+}:$184.0768, found 184.0767.

\section{$N$-(4-methoxyphenyl)acetamide: ${ }^{4}$}

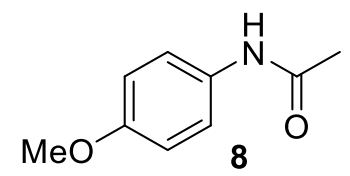

Reaction time: $2.5 \mathrm{~h} ; \mathrm{R}_{f}=0.5$ (EtOAc:Pet. Ether, 1:1). White solid; $48 \mathrm{mg}$, 96\%, mp 130-133 ${ }^{\circ} \mathrm{C}$;

${ }^{1} \mathrm{H}$ NMR (400 MHz, $\left.\mathrm{CDCl}_{3}\right): \delta 7.39(\mathrm{~d}, J=8.8 \mathrm{~Hz}, 2 \mathrm{H}), 7.33(\mathrm{bs}, 1 \mathrm{H}), 6.85(\mathrm{~d}, J=8.8 \mathrm{~Hz}, 2 \mathrm{H})$, 3.79 (s, 3H), 2.15 (s, 3H); ${ }^{13} \mathrm{C}$ NMR (100 MHz, $\left.\mathrm{CDCl}_{3}\right): 168.3,156.4,130.9,121.9,114.1,55.5$, 24.3; GC-MS $\left(\mathrm{M}^{+}\right) 165$. 


\section{$N$-(4-hydroxyphenyl)acetamide: ${ }^{3}$}

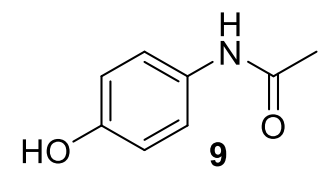

Reaction time: 45 min.; $\mathrm{R}_{f}=0.5$ (EtOAc:Pet. Ether, 1:1). White solid; $33 \mathrm{mg}, 66 \%$, mp 166-168

${ }^{\circ} \mathrm{C} ;{ }^{1} \mathrm{H}$ NMR (400 MHz, Acetone- $\left.d_{6}\right): \delta 8.96(\mathrm{bs}, 1 \mathrm{H}), 8.21(\mathrm{~s}, 1 \mathrm{H}), 7.43(\mathrm{~d}, J=9.0 \mathrm{~Hz}, 2 \mathrm{H}), 6.74$ $(\mathrm{d}, J=9.0 \mathrm{~Hz}, 2 \mathrm{H}), 2.02(\mathrm{~s}, 3 \mathrm{H}) ;{ }^{13} \mathrm{C}$ NMR (100 MHz, Acetone- $\left.d_{6}\right): 168.2,154.1,132.7,121.6$, 115.8, 24.0; GC-MS $\left(\mathrm{M}^{+}\right) 165$.

\section{$N$-(2-hydroxyphenyl)acetamide: ${ }^{6}$}

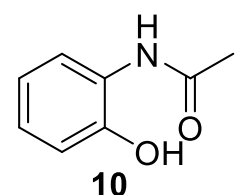

Reaction time: 30 min.; $\mathrm{R}_{f}=0.5$ (EtOAc:Pet. Ether, 1:1). White solid; $32.3 \mathrm{mg}, 65 \%$, mp 206$209{ }^{\circ} \mathrm{C} ;{ }^{1} \mathrm{H}$ NMR (400 MHz, DMSO-d $): \delta 9.74(\mathrm{~s}, 1 \mathrm{H}), 9.30(\mathrm{~s}, 1 \mathrm{H}), 7.67(\mathrm{~d}, J=7.6 \mathrm{~Hz}, 1 \mathrm{H})$, $6.93(\mathrm{t}, J=7.6 \mathrm{~Hz}, 1 \mathrm{H}), 6.85(\mathrm{~d}, J=7.1 \mathrm{~Hz}, 1 \mathrm{H}), 6.75(\mathrm{t}, J=7.6 \mathrm{~Hz}, 1 \mathrm{H}), 2.09(\mathrm{~s}, 3 \mathrm{H}) ;{ }^{13} \mathrm{C} \mathrm{NMR}$ (100 MHz, DMSO- $\left.d_{6}\right): \delta 169.2,148.1,126.6,124.8,122.6,119.2,116.1,23.8 ; \quad$ HRMS-ESI $(m / z)$ calcd $\left[\mathrm{C}_{8} \mathrm{H}_{9} \mathrm{O}_{2} \mathrm{~N}+\mathrm{H}\right]^{+}:$152.0706, found 152.0704. 


\section{$N$-(6-methoxynaphthalen-2-yl)acetamide: ${ }^{7}$}

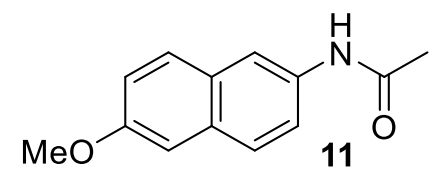

Reaction time: 20 min.; $\mathrm{R}_{f}=0.5$ (EtOAc:Pet. Ether, 2:3). White solid; $45 \mathrm{mg}$, 90\%, mp 162-163 ${ }^{\circ} \mathrm{C} ;{ }^{1} \mathrm{H}$ NMR $\left(400 \mathrm{MHz}, \mathrm{CDCl}_{3}\right): \delta 8.07(\mathrm{~s}, 1 \mathrm{H}), 7.67$ (d, J=8.8 Hz, 2H), 7.48 (bs, 1H), 7.44 (dd, $J=8.8,2.0 \mathrm{~Hz}, 1 \mathrm{H}), 7.14(\mathrm{dd}, J=8.8,2.4 \mathrm{~Hz}, 1 \mathrm{H}), 7.09(\mathrm{~d}, J=2.2 \mathrm{~Hz}, 1 \mathrm{H}), 3.91(\mathrm{~s}, 3 \mathrm{H}), 2.22$ (s, 3H); ${ }^{13} \mathrm{C}$ NMR (100 MHz, $\left.\mathrm{CDCl}_{3}\right): \delta 168.4,157.1,133.5,131.7,129.1,127.4,120.5,119.2$, 117.1, 105.6, 55.3, 24.6; GC-MS $\left(\mathrm{M}^{+}\right) 215$.

\section{$N$-(1-(methylsulfonyl)-1H-pyrrol-2-yl)acetamide:}

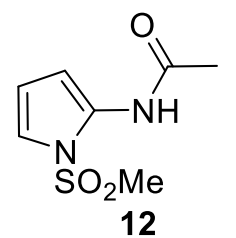

Reaction time: $1.5 \mathrm{~h} ; \mathrm{R}_{f}=0.3$ (EtOAc:Pet. Ether, 1:1). Brown solid; $23.7 \mathrm{mg}, 47 \%$, mp 125-127 ${ }^{\circ} \mathrm{C} ;{ }^{1} \mathrm{H}$ NMR $\left(500 \mathrm{MHz}, \mathrm{CDCl}_{3}\right): \delta 8.14(\mathrm{bs}, 1 \mathrm{H}), 6.87(\mathrm{dd}, J=1.5 \mathrm{~Hz}, 1 \mathrm{H}), 6.53(\mathrm{~d}, J=1.2 \mathrm{~Hz}$, 1H), $6.29(\mathrm{t}, J=3.4 \mathrm{~Hz}, 3.7 \mathrm{~Hz}, 1 \mathrm{H}), 3.15(\mathrm{~s}, 3 \mathrm{H}), 2.19(\mathrm{~s}, 3 \mathrm{H}) ;{ }^{13} \mathrm{C} \mathrm{NMR}\left(125 \mathrm{MHz}, \mathrm{CDCl}_{3}\right): \delta$ 168.1, 127.3, 117.0, 112.3, 105.6, 42.2, 23.8; HRMS-ESI $(m / z)$ calcd $\left[\mathrm{C}_{7} \mathrm{H}_{10} \mathrm{O}_{3} \mathrm{~N}_{2} \mathrm{~S}+\mathrm{Na}\right]^{+}$: 225.0304, found 225.0307. 


\section{N,2-bis(3,4-dimethoxyphenyl)acetamide: ${ }^{8}$}

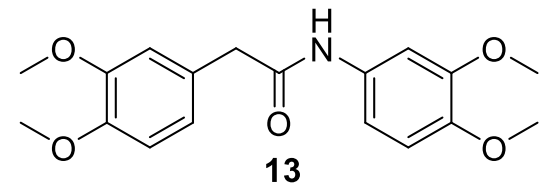

Reaction time: 3 h; $\mathrm{R}_{f}$ : 0.5 (EtOAc:Pet. Ether, 1:1); Silver white Solid; 32.5 mg, 65\%; mp 140$142{ }^{\circ} \mathrm{C} ;{ }^{1} \mathrm{H}$ NMR $\left(400 \mathrm{MHz}, \mathrm{CDCl}_{3}\right) \delta 7.27(\mathrm{~s}, 1 \mathrm{H}), 7.11(\mathrm{~s}, 1 \mathrm{H}), 6.90-8.86(\mathrm{~m}, 2 \mathrm{H}), 6.84(\mathrm{~s}$, 1H), $6.76(\mathrm{~s}, 2 \mathrm{H}), 3.91(\mathrm{~s}, 3 \mathrm{H}), 3.90(\mathrm{~s}, 3 \mathrm{H}), 3.85(\mathrm{~s}, 3 \mathrm{H}), 3.84(\mathrm{~s}, 3 \mathrm{H}), 3.67(\mathrm{~s}, 2 \mathrm{H}) ;{ }^{13} \mathrm{C} \mathrm{NMR}$ $\left(100 \mathrm{MHz}, \mathrm{CDCl}_{3}\right) \delta 169.3,149.4,139.3,149.0,148.6,145.9,131.2,126.8,121.8,112.5,111.7$, 111.6, 111.2, 104.8, 56.1, 55.9, 44.3; GC-MS $\left(\mathrm{M}^{+}\right) 331$.

\section{Ethyl 4-((3,4-dimethoxyphenyl)amino)-4-oxobutanoate:}

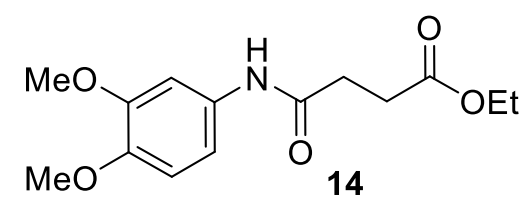

Reaction time: $10 \mathrm{~min}$; $\mathrm{R}_{f}$ : 0.5 (EtOAc:Pet. Ether, 1:1); Silver white Solid; $27 \mathrm{mg}, 54 \%$; mp 110$113{ }^{\circ} \mathrm{C} ;{ }^{1} \mathrm{H}$ NMR $\left(400 \mathrm{MHz}, \mathrm{CDCl}_{3}\right): \delta 7.62(\mathrm{~s}, 1 \mathrm{H}), 7.34(\mathrm{~s}, 1 \mathrm{H}), 6.86(\mathrm{dd}, J=7.8,2.2 \mathrm{~Hz}, 1 \mathrm{H})$, $6.78(\mathrm{~d}, J=8.6 \mathrm{~Hz}, 1 \mathrm{H}), 4.17(\mathrm{q}, J=7.1 \mathrm{~Hz}, 2 \mathrm{H}), 3.87(\mathrm{~s}, 3 \mathrm{H}), 3.85(\mathrm{~s}, 3 \mathrm{H}), 2.75(\mathrm{t}, J=6.4 \mathrm{~Hz}$, 2H), $2.64(\mathrm{t}, J=6.4 \mathrm{~Hz}, 2 \mathrm{H}), 1.27(\mathrm{t}, J=7.1 \mathrm{~Hz}, 3 \mathrm{H}) ;{ }^{13} \mathrm{C}$ NMR $\left(100 \mathrm{MHz}, \mathrm{CDCl}_{3}\right): \delta 173.2$, 169.6, 149.0, 145.7, 131.5, 111.6, 111.2, 104.8, 60.9, 56.1, 55.9, 32.0, 29.5, 14.1; HRMS-ESI $(m / z)$ calcd $\left[\mathrm{C}_{14} \mathrm{H}_{19} \mathrm{O}_{5} \mathrm{~N}+\mathrm{H}\right]^{+}:$282.1336, found 282.1328 . 


\section{$N$-phenylbenzamide: ${ }^{9}$}

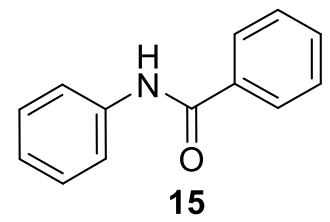

Reaction time: 9 h; Rf: 0.5 (EtOAc:Pet. Ether, 2:3); white Solid; 41.7 mg, 83\%; mp 161-163 ${ }^{\circ} \mathrm{C}$;

${ }^{1} \mathrm{H}$ NMR (500 MHz, $\left.\mathrm{CDCl}_{3}\right): \delta 7.88(\mathrm{bs}, 1 \mathrm{H}), 7.88(\mathrm{~d}, J=7.3 \mathrm{~Hz}, 2 \mathrm{H}), 7.66(\mathrm{~d}, J=7.6 \mathrm{~Hz}, 2 \mathrm{H})$, $7.56(\mathrm{t}, J=7.0 \mathrm{~Hz}, 1 \mathrm{H}), 7.49(\mathrm{t}, J=7.0,7.6 \mathrm{~Hz}, 2 \mathrm{H}), 7.38(\mathrm{t}, J=7.02,7.3 \mathrm{~Hz}, 2 \mathrm{H}), 7.17(\mathrm{t}, J=$

7.0, $7.3 \mathrm{~Hz}, 1 \mathrm{H}) ;{ }^{13} \mathrm{C} \mathrm{NMR}\left(125 \mathrm{MHz}, \mathrm{CDCl}_{3}\right): \delta$ 165.8. 137.9, 135.0, 131.8, 129.1, 128.8, 127.0, 124.6, 120.2; GC-MS $\left(\mathrm{M}^{+}\right) 197$.

$N$-(2-hydroxyphenyl)benzamide: ${ }^{10}$<smiles>O=C(Nc1ccccc1O)c1ccccc1</smiles>

Reaction Time: 05 h; $\mathrm{R}_{f}$ : 0.5 (EtOAc:Pet. Ether, 1:4); White Solid; $30 \mathrm{mg}$, 60\%; mp 135-137 ${ }^{\circ} \mathrm{C}$; ${ }^{1} \mathrm{H}$ NMR $\left(500 \mathrm{MHz}, \mathrm{DMSO}-d_{6}\right) \delta 9.80(\mathrm{~s}, 1 \mathrm{H}), 9.52(\mathrm{~s}, 1 \mathrm{H}), 7.96(\mathrm{~d}, J=7.6 \mathrm{~Hz}, 2 \mathrm{H}), 7.67(\mathrm{~d}, J$ $=7.6 \mathrm{~Hz}, 1 \mathrm{H}), 7.61-7.50(\mathrm{~m}, 3 \mathrm{H}), 7.10-6.80(\mathrm{~m}, 3 \mathrm{H}) ;{ }^{13} \mathrm{C} \mathrm{NMR}\left(125 \mathrm{MHz}, \mathrm{DMSO}-d_{6}\right) \delta 165.5$, 149.6, 134.5, 131.9, 128.7, 127.7, 126.0, 124.4, 119.3, 116.2; GC-MS $\left(\mathrm{M}^{+}\right) 213$. 


\section{Benzo[d][1,3]dioxole-5-carbonitrile: ${ }^{11}$}

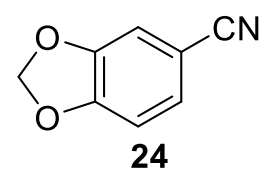

Reaction time: $1 \mathrm{~h} ; \mathrm{R}_{f}=0.5$ (EtOAc:Pet. Ether, 1:5). White solid; $38 \mathrm{mg}, 85 \%$, mp 94-97 ${ }^{\circ} \mathrm{C} ;{ }^{1} \mathrm{H}$ NMR (400 MHz, $\left.\mathrm{CDCl}_{3}\right): \delta 7.22(\mathrm{dd}, J=8.1 \mathrm{~Hz}, 1.5 \mathrm{~Hz}, 1 \mathrm{H}), 7.04(\mathrm{~d}, J=1.5 \mathrm{~Hz}, 1 \mathrm{H}), 6.87(\mathrm{~d}, J$ $=8.1 \mathrm{~Hz}, 1 \mathrm{H}), 6.08(\mathrm{~s}, 2 \mathrm{H}) ;{ }^{13} \mathrm{C} \mathrm{NMR}\left(100 \mathrm{MHz}, \mathrm{CDCl}_{3}\right): \delta 151.5,148.0,128.2,118.9,111.4$, 109.1, 104.9, 102.3; LC-MS (M+Na) 170.

\section{Azepan-2-one: ${ }^{12}$}

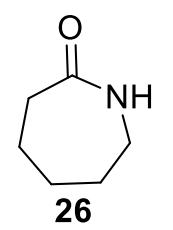

Reaction Time: $15 \mathrm{~min}$. at $70{ }^{\circ} \mathrm{C} \mathrm{R}_{f}: 0.5$ (MeOH: DCM, 1:20); White Solid; $16 \mathrm{mg}, 32 \%$; mp 68$70{ }^{\circ} \mathrm{C} ;{ }^{1} \mathrm{H}$ NMR $\left(400 \mathrm{MHz}, \mathrm{CDCl}_{3}\right) \delta 6.3(\mathrm{bs}, 1 \mathrm{H}), 3.25-3.15(\mathrm{~m}, J=2 \mathrm{H}), 2.50-2.45(\mathrm{~m}, 2 \mathrm{H})$, 1.80-1.60 (m, 6H); ${ }^{13} \mathrm{C}$ NMR (100 MHz, $\left.\mathrm{CDCl}_{3}\right) \delta 179.1,42.9,36.6,30.6,29.7,23.2$; LC-MS $(\mathrm{M}+\mathrm{H}) 114$. 


\section{Experimental procedures and data for Scheme 5 (Isocryptolepine Synthesis):}

Synthesis of $N$-phenyl-1-(phenylsulfonyl)-1H-indole-3-carboxamide (29):

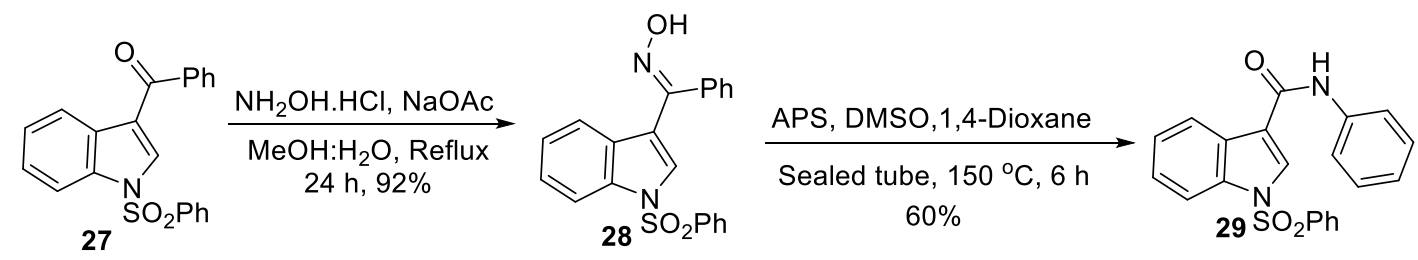

\section{(E)-phenyl(1-(phenylsulfonyl)-1H-indol-3-yl)methanone oxime 28:}

A two necked round bottom flask containing the solution of benzoyl compound 27 ( $1 \mathrm{~g}, 2.76$ mmol, 1 equiv), $\mathrm{NH}_{2} \mathrm{OH} . \mathrm{HCl}$ (4.8 g, 69.17 mmol, 25 equiv) and $\mathrm{NaOAc}$ (5 g, 60.9 mmol, 22 equiv) in $\mathrm{MeOH}: \mathrm{H}_{2} \mathrm{O}(50 \mathrm{~mL}: 15 \mathrm{~mL})$ was heated at $90{ }^{\circ} \mathrm{C}$ for 24 hours. After completion of the reaction, methanol was evaporated in vaccuo and the residue was extracted with ethyl acetate (20 $\mathrm{mL}$ ). Organic layer was dried over sodium sulfate, concentrated and the crude product was purified by recrystallization in methanol to provide ketoxime 28 (959 $\mathrm{mg}, 92 \%)$ as a white solid.

Reaction time: $24 \mathrm{~h} ; \mathrm{R}_{f}=0.3$ (EtOAc:Pet. Ether, 3:7). White solid; mp 186-187 ${ }^{\circ} \mathrm{C} ;{ }^{1} \mathrm{H}$ NMR $\left(400 \mathrm{MHz}, \mathrm{DMSO}_{-} \mathrm{d}_{6}\right): \delta 11.76(\mathrm{~s}, 1 \mathrm{H}), 8.09(\mathrm{~s}, 1 \mathrm{H}), 8.06(\mathrm{~d}, J=7.7 \mathrm{~Hz}, 2 \mathrm{H}), 8.00(\mathrm{~d}, J=8.3$ $\mathrm{Hz}, 1 \mathrm{H}), 7.73(\mathrm{t}, J=7.3 \mathrm{~Hz}, 1 \mathrm{H}), 7.63(\mathrm{t}, J=7.8,7.3 \mathrm{~Hz}, 2 \mathrm{H}), 7.42-7.34(\mathrm{~m}, 6 \mathrm{H}), 7.16(\mathrm{t}, J=$ 7.8, $7.3 \mathrm{~Hz}, 1 \mathrm{H}), 6.96(\mathrm{t}, J=7.8 \mathrm{~Hz}, 1 \mathrm{H}) ;{ }^{13} \mathrm{C}$ NMR $\left(100 \mathrm{MHz}, \mathrm{DMSO}-\mathrm{d}_{6}\right): \delta 148.5,137.0$, $136.4,135.1,134.0,130.2,129.3,129.1,128.7,128.1,127.3,127.1,125.2,123.7,121.9,114.5$, 113.4; HRMS-ESI $(\mathrm{m} / \mathrm{z})$ calcd $\left[\mathrm{C}_{21} \mathrm{H}_{16} \mathrm{O}_{3} \mathrm{~N}_{2} \mathrm{~S}+\mathrm{H}\right]^{+}: 377.0954$, found 377.0943 . 


\section{$N$-Phenyl-1-(phenylsulfonyl)-1H-indole-3-carboxamide $29:^{13}$}

The reaction mixture containing ketoxime 28 (500 mg, $1.3 \mathrm{mmol}, 1$ equiv), APS (606 mg, 2.65 mmol, 2 equiv) and DMSO (0.56 mL, $7.96 \mathrm{mmol}, 6$ equiv) in 1,4-dioxane (10 mL) was heated at $150{ }^{\circ} \mathrm{C}$ for 6 hours in a glass tube sealed with Teflon cap. After completion of the reaction, 1,4dioxane was evaporated under vacuum and the residue was dissolved in ethyl acetate $(50 \mathrm{~mL})$. The organic layer was washed with brine $(10 \times 2 \mathrm{~mL})$ and dried over sodium sulfate. Evaporation of ethyl acetate under vacuum followed by flash column chromatography of the residue with ethyl acetate: petroleum ether (3:7) gave (300 mg, 60\%) of compound 29.

Reaction time: $6 \mathrm{~h} ; \mathrm{R}_{f}=0.5$ (EtOAc:Pet. Ether, 3:7). White solid; mp 172-174 ${ }^{\circ} \mathrm{C} ;{ }^{1} \mathrm{H}$ NMR $(500$ $\left.\mathrm{MHz}, \mathrm{CDCl}_{3}\right): \delta 8.17(\mathrm{~s}, 1 \mathrm{H}), 8.12(\mathrm{~d}, J=7.6,1 \mathrm{H}), 8.01(\mathrm{~d}, J=8.4,1 \mathrm{H}), 7.94(\mathrm{~d}, J=7.6,2 \mathrm{H})$, $7.80(\mathrm{bs}, 1 \mathrm{H}), 7.65(\mathrm{~d}, J=8.0,2 \mathrm{H}), 7.59(\mathrm{t}, J=7.6,1 \mathrm{H}), 7.48(\mathrm{t}, J=7.6,2 \mathrm{H}), 7.43-7.35(\mathrm{~m}, 4 \mathrm{H})$, $7.17(\mathrm{t}, J=7.3,1 \mathrm{H}) ;{ }^{13} \mathrm{C} \mathrm{NMR}\left(125 \mathrm{MHz}, \mathrm{CDCl}_{3}\right): \delta 161.5,137.62,137.56,135.0,134.5,129.6$, 129.2, 127.8, 127.4, 127.0, 125.8, 124.63, 124.55, 121.7, 120.2, 118.0, 113.5; HRMS-ESI $(\mathrm{m} / \mathrm{z})$ calcd $\left[\mathrm{C}_{21} \mathrm{H}_{16} \mathrm{O}_{3} \mathrm{~N}_{2} \mathrm{~S}+\mathrm{Na}\right]^{+}:$399.0774, found 399.0760.

\section{$N$-phenylbenzo[4,5]isothiazolo[2,3-a]indole-11-carboxamide 5,5-dioxide (34):}

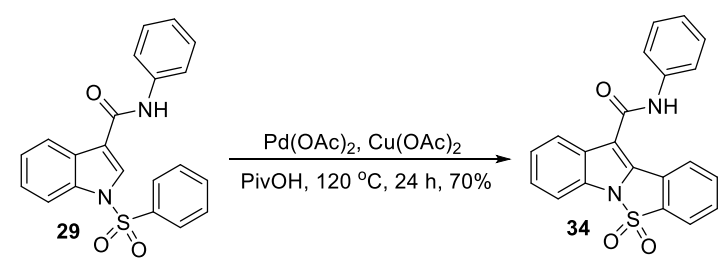

Amide 29 (200 mg, $0.53 \mathrm{mmol}, 1$ equiv), palladium acetate (23 mg, $0.1 \mathrm{mmol}, 0.2$ equiv) and copper acetate (96 mg, $0.53 \mathrm{mmol}, 1$ equiv) were taken in a schlenk tube equipped with a 
magnetic stirring bar. It was kept under reduced pressure for some time, flushed with oxygen and kept under oxygen balloon pressure. After the addition of $1 \mathrm{~mL}$ of pivalic acid the reaction was heated at $120{ }^{\circ} \mathrm{C}$ for $24 \mathrm{~h}$. It was then allowed to attain room temperature and ethyl acetate (15 $\mathrm{mL}$ ) was added. The ethyl acetate layer was washed with aqueous saturated sodium bicarbonate (5 mL x 3), dried over sodium sulfate and concentrated under vacuum. Flash column chromatography using ethyl acetate: petroleum ether (3:7) yielded $140 \mathrm{mg}$ of pure compound 34 (70\%).

Reaction time: $24 \mathrm{~h} ; \mathrm{R}_{f}=0.5$ (EtOAc:Pet. Ether, 3:7). White solid; mp 202-207 ${ }^{\circ} \mathrm{C} ;{ }^{1} \mathrm{H}$ NMR $\left(400 \mathrm{MHz}, \mathrm{CDCl}_{3}\right): \delta 10.71(\mathrm{~s}, 1 \mathrm{H}), 8.31(\mathrm{~d}, J=7.8,1 \mathrm{H}), 8.22(\mathrm{~d}, J=7.8,1 \mathrm{H}), 7.94-790(\mathrm{~m}$, 2H), 7.81-7.76 (m, 4H), $7.55(\mathrm{t}, J=7.8,1 \mathrm{H}), 7.47-7.39(\mathrm{~m}, 3 \mathrm{H}), 7.17(\mathrm{t}, J=7.3,1 \mathrm{H}) ;{ }^{13} \mathrm{C}$ NMR $\left(100 \mathrm{MHz}, \mathrm{CDCl}_{3}\right): \delta 161.1,138.9,137.5,135.6,132.4,131.5,131.1,130.4,129.1,127.1,125.8$, $125.5, \quad 124.5, \quad 124.4, \quad 123.4, \quad 123.0, \quad 120.4, \quad 113.1, \quad 111.5 ; \quad$ HRMS-ESI $(m / z) \quad c a l c d$ $\left[\mathrm{C}_{21} \mathrm{H}_{14} \mathrm{O}_{3} \mathrm{~N}_{2} \mathrm{~S}+\mathrm{Na}\right]^{+}:$397.0617, found 397.0606.

\section{Formal Total synthesis of Isocryptolepine from MOM-protected indole:}

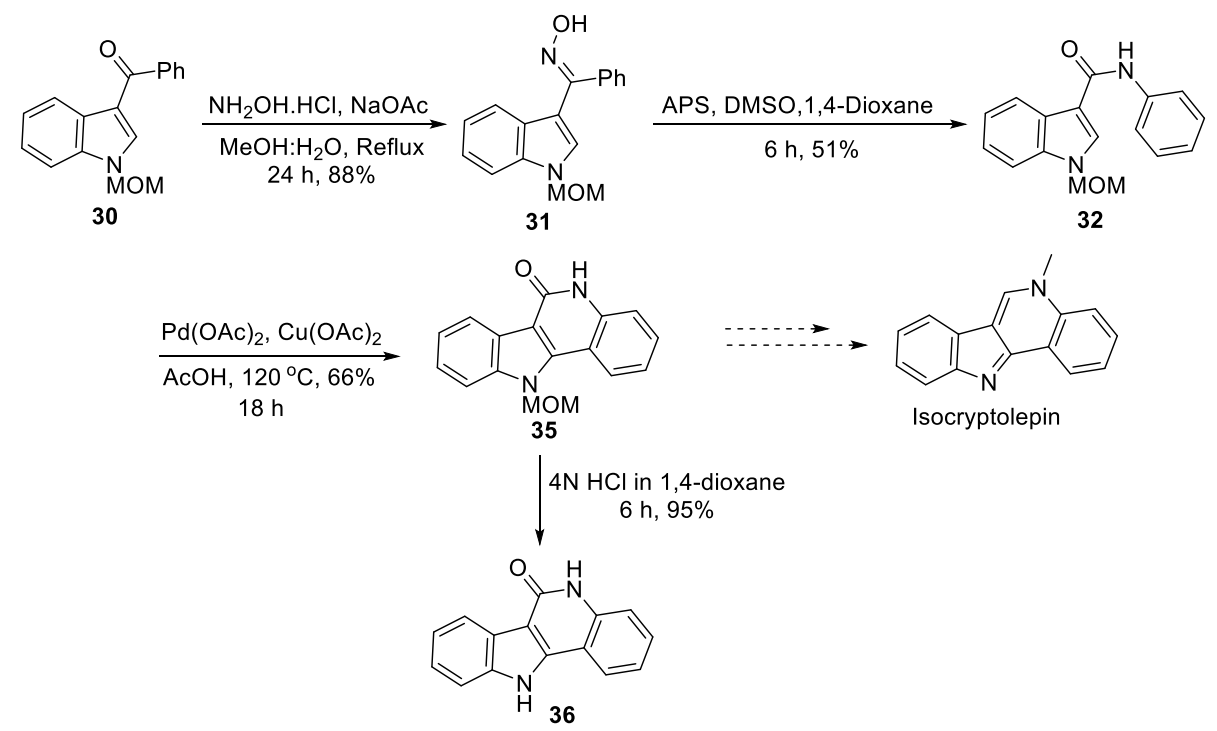




\section{(1-(methoxymethyl)-1H-indol-3-yl)(phenyl)methanone (30):}

In a two necked round bottom flask containing the solution of (1H-indol-3-yl)(phenyl)methanone (200 mg, $0.90 \mathrm{mmol}, 1$ equiv) in DMF was added $\mathrm{NaH}\left(65 \mathrm{mg}, 2.7 \mathrm{mmol}, 3\right.$ equiv) at $0{ }^{\circ} \mathrm{C}$ and the reaction mixture was stirred for another 10 minutes. MOM chloride $(0.1 \mathrm{~mL}, 1.35 \mathrm{mmol}, 1.5$ equiv) was added and the reaction mixture was stirred at $\mathrm{rt}$ for $6 \mathrm{~h}$. After the completion of the reaction, ice was added followed by extraction with ethyl acetate $(10 \mathrm{~mL}$ x 2$)$. The organic layer was washed with brine $(10 \mathrm{~mL} \times 2)$ and dried over sodium sulfate. Concentration of organic layer under vacuum followed by column chromatography ethyl acetate: petroleum ether (3:7) yielded MOM protected ketone $\mathbf{3 0}$ in $216 \mathrm{mg}$.

Reaction time: $6 \mathrm{~h} ; \mathrm{R}_{f}=0.4$ (EtOAc:Pet. Ether, 3:7). White solid; 90\%, mp 97-99 ${ }^{\circ} \mathrm{C} ;{ }^{1} \mathrm{H}$ NMR $\left(400 \mathrm{MHz}, \mathrm{CDCl}_{3}\right): \delta 8.45-8.42(\mathrm{~m}, 1 \mathrm{H}), 7.85(\mathrm{~d}, J=7.3 \mathrm{~Hz}, 2 \mathrm{H}), 7.66(\mathrm{~s}, 1 \mathrm{H}), 7.60-7.55(\mathrm{~m}$, $2 \mathrm{H}), 7.51(\mathrm{t}, J=7.3 \mathrm{~Hz}, 2 \mathrm{H}), 7.41-7.36(\mathrm{~m}, 2 \mathrm{H}), 5.50(\mathrm{~s}, 2 \mathrm{H}), 3.30(\mathrm{~s}, 3 \mathrm{H}) ;{ }^{13} \mathrm{C} \mathrm{NMR}(100 \mathrm{MHz}$, $\left.\mathrm{CDCl}_{3}\right): \delta 191.1,140.6,136.9,136.7,131.4,128.7,128.4,127.5,124.2,123.2,122.8,166.7$, 110.3, 78.1, 56.3; HRMS-ESI $(m / z)$ calcd $\left[\mathrm{C}_{17} \mathrm{H}_{15} \mathrm{O}_{2} \mathrm{~N}+\mathrm{Na}\right]^{+}: 288.0995$, found 288.0988.

\section{(E)-(1-(methoxymethyl)-1H-indol-3-yl)(phenyl)methanone oxime (31):}

In a round bottom flask containing MOM ketone 30 (200 $\mathrm{mg}, 0.75 \mathrm{mmol}, 1$ equiv), hydroxylamine hydrochloride ( $1.3 \mathrm{~g}, 18.8 \mathrm{~mol}, 25$ equiv) and sodium acetate $(1.54 \mathrm{~g}, 18.8 \mathrm{~mol}$, 25 equiv) was added $13 \mathrm{~mL}$ of methanol:water (10:3) and the resulting reaction mixture was heated at $90{ }^{\circ} \mathrm{C}$ for $24 \mathrm{~h}$. After completion of the reaction as indicated by thin layer chromatography, methanol was evaporated followed by the addition of ethyl acetate $(20 \mathrm{~mL})$. 
The organic layer was washed with cold water $(10 \mathrm{~mL}$ x 2), dried over sodium sulfate and concentrated under vacuum. Flash column chromatography ethyl acetate: petroleum ether (3:7) afforded ketoxime 31 in 186 mg yield.

Reaction time: $24 \mathrm{~h} ; \mathrm{R}_{f}=0.3$ (EtOAc:Pet. Ether, 3:7). White solid; 88\%, mp 155-157 ${ }^{\circ} \mathrm{C} ;{ }^{1} \mathrm{H}$ NMR (500 MHz, DMSO-d $): \delta 11.39(\mathrm{~s}, 1 \mathrm{H}), 8.07(\mathrm{~s}, 1 \mathrm{H}), 7.60(\mathrm{~d}, J=8.0 \mathrm{~Hz}, 1 \mathrm{H}), 7.46-7.39$ (m, 5H), $7.16(\mathrm{t}, J=7.3 \mathrm{~Hz}, 1 \mathrm{H}), 6.94(\mathrm{t}, J=7.3 \mathrm{~Hz}, 1 \mathrm{H}), 6.77(\mathrm{~d}, J=7.6 \mathrm{~Hz}, 1 \mathrm{H}), 5.61(\mathrm{~s}, 2 \mathrm{H})$, $3.21(\mathrm{~s}, 3 \mathrm{H}) ;{ }^{13} \mathrm{C}$ NMR $\left(125 \mathrm{MHz}, \mathrm{DMSO}-d_{6}\right): \delta 150.3,137.9,135.7,133.3,128.8,128.4,128.2$, 127.2, 122.0, 121.3, 120.3, 110.8, 107.0, 76.9, 55.6; HRMS-ESI $(m / z)$ calcd $\left[\mathrm{C}_{17} \mathrm{H}_{16} \mathrm{O}_{2} \mathrm{~N}_{2}+\mathrm{H}\right]^{+}$: 281.1285 , found 281.1281.

\section{1-(methoxymethyl)- $N$-phenyl-1H-indole-3-carboxamide (32):}

A Schlenk tube containing ketoxime 31 (100 mg, $0.35 \mathrm{mmol}, 1$ equiv) and ammonium peroxysulfate (122 mg, $0.53 \mathrm{mmol}, 1.5$ equiv) was kept under reduced pressure and flushed with Argon, followed by the addition of 1,4-dioxane (4 mL) and DMSO (0.15 mL, $2.14 \mathrm{mmol}, 6$ equiv). The reaction mixture was heated at $100{ }^{\circ} \mathrm{C}$ for $6 \mathrm{~h}$. After completion of the reaction, 1,4dioxane was evaporated and $10 \mathrm{~mL}$ ethyl acetate was added. The organic layer was washed with brine ( $5 \mathrm{~mL} \mathrm{X} \mathrm{2),} \mathrm{dried} \mathrm{over} \mathrm{sodium} \mathrm{sulfate} \mathrm{and} \mathrm{concentrated.} \mathrm{Column} \mathrm{chromatography} \mathrm{ethyl}$ acetate: petroleum ether (2:3) of the residue afforded $51 \mathrm{mg}$ of $\mathbf{3 2}$.

Reaction time: $6 \mathrm{~h} ; \mathrm{R}_{f}=0.5$ (EtOAc:Pet. Ether, 2:3). White solid; $51 \mathrm{mg}, 51 \%, \mathrm{mp} 78-82{ }^{\circ} \mathrm{C} ;{ }^{1} \mathrm{H}$ NMR (400 MHz, $\left.\mathrm{CDCl}_{3}\right): \delta 8.10-8.08(\mathrm{~m}, 1 \mathrm{H}), 7.85(\mathrm{~s}, 1 \mathrm{H}), 7.84$ (bs, 1H), 7.68(d, J=8.1 Hz, 2H), 7.55-7.55 (m, 1H), 7.40-734 (m, 4H), $7.14(\mathrm{t}, J=7.3 \mathrm{~Hz}, 1 \mathrm{H}), 5.45(\mathrm{~s}, 2 \mathrm{H}), 3.26(\mathrm{~s}, 3 \mathrm{H})$; ${ }^{13} \mathrm{C}$ NMR $\left(100 \mathrm{MHz}, \mathrm{CDCl}_{3}\right): \delta 163.0,138.2,136.7,131.5,129.1,125.9,124.1,123.4,122.4$, 
120.3, 120.1, 112.5, 111.0, 78.0, 56.2; HRMS-ESI $(\mathrm{m} / \mathrm{z})$ calcd $\left[\mathrm{C}_{17} \mathrm{H}_{16} \mathrm{O}_{2} \mathrm{~N}_{2}+\mathrm{Na}\right]^{+}:$303.1104, found 303.1102.

\section{1-(methoxymethyl)-5,11-dihydro-6H-indolo[3,2-c]quinolin-6-one (35): ${ }^{14}$}

To a flame dried schlenk tube kept under oxygen balloon were added amide 32 (40 mg, 0.14 mmol, 1 eq), palladium acetate $(6.5 \mathrm{mg}, 0.02 \mathrm{mmol}, 0.2 \mathrm{eq})$ and copper acetate $(26 \mathrm{mg}, 0.14$ mmol, 1 eq). The schlenk tube was evacuated and charged with $1 \mathrm{~mL}$ of acetic acid under oxygen atmosphere. The reaction mixture was heated at $110{ }^{\circ} \mathrm{C}$ for $18 \mathrm{~h}$. It was then allowed to come to the room temperature and ice water $(5 \mathrm{~mL})$ was added with stirring. The reaction mixture was extracted with ethyl acetate $(10 \mathrm{~mL} \mathrm{X} \mathrm{2)}$ and the combined organic layer was washed with aqueous saturated sodium bicarbonate $(5 \mathrm{~mL} \mathrm{X} \mathrm{2).} \mathrm{The} \mathrm{organic} \mathrm{layer} \mathrm{was} \mathrm{dried} \mathrm{over}$ sodium sulfate and purified by column chromatography with ethyl acetate:petroleum ether $(1: 1)$ to furnish tetracyclic amide $\mathbf{3 5}$ (26 $\mathrm{mg}, 66 \%)$.

Reaction time: $18 \mathrm{~h} ; \mathrm{R}_{f}=0.3$ (EtOAc:Pet. Ether, 2:3). White solid; $26 \mathrm{mg}, 66 \%$, mp 272-274 ${ }^{\circ} \mathrm{C}$; ${ }^{1} \mathrm{H}$ NMR (400 MHz, $\left.\mathrm{CDCl}_{3}\right): \delta 11.70(\mathrm{bs}, 1 \mathrm{H}), 8.67(\mathrm{~d}, J=7.3 \mathrm{~Hz}, 1 \mathrm{H}), 8.40(\mathrm{~d}, J=8.3 \mathrm{~Hz}, 1 \mathrm{H})$, $7.67(\mathrm{~d}, J=8.1 \mathrm{~Hz}, 1 \mathrm{H}), 7.65(\mathrm{~d}, J=8.1 \mathrm{~Hz}, 1 \mathrm{H}), 7.58(\mathrm{t}, J=7.3 \mathrm{~Hz}, 7.5 \mathrm{~Hz}, 1 \mathrm{H}), 7.51(\mathrm{t}, J=7.3$ Hz, 7.1 Hz, 1H), 7.46 (t, J= 7.5 Hz, 7.1 Hz, 1H), 7.35 (t, J= $7.5 \mathrm{~Hz}, 1 \mathrm{H}), 5.95$ (s, 2H), 3.51 (s, $3 \mathrm{H}) ;{ }^{13} \mathrm{C}$ NMR $\left(100 \mathrm{MHz}, \mathrm{CDCl}_{3}\right): \delta 161.4,141.4,140.1,138.2,129.2,125.0,124.3,123.6$, 122.5, 122.46, 117.2, 112.8, 109.0, 75.3, 56.3; HRMS-ESI $(m / z)$ calcd $\left[\mathrm{C}_{17} \mathrm{H}_{15} \mathrm{O}_{2} \mathrm{~N}_{2}+\mathrm{H}\right]^{+}$: 279.1128 , found 279.1126 . 


\section{5,11-dihydro-6H-indolo[3,2-c]quinolin-6-one $(36):^{14}$}

In a round bottom flask, the solution of MOM-protected amide 35 (20 mg, $0.07 \mathrm{mmol}, 1 \mathrm{eq})$ and $4 \mathrm{~N} \mathrm{HCl}$ in 1,4-dioxane $(1 \mathrm{~mL})$ was refluxed for $6 \mathrm{~h}$. After completion of the reaction as indicated by thin layered chromatography, solvent was evaporated under vacuum and ethyl acetate (10 $\mathrm{mL}$ ) was added. The ethyl acetate layer was washed with aqueous saturated sodium bicarbonate ( $5 \mathrm{~mL}$ X 2), dried over sodium sulfate and concentrated. Purification of the residue by flash column chromatography using ethyl acetate:petroleum ether (1:1) afforded $16 \mathrm{mg}$ of $\mathbf{3 6}$.

Reaction time: $16 \mathrm{~h} ; \mathrm{R}_{f}=0.4$ (EtOAc:Pet. Ether, 1:1). White solid; $16 \mathrm{mg}, 95 \%, \mathrm{mp} 337-339{ }^{\circ} \mathrm{C}$; ${ }^{1} \mathrm{H}$ NMR (400 MHz, DMSO- $\left.d_{6}\right): \delta 12.57(\mathrm{~s}, 1 \mathrm{H}), 11.43(\mathrm{~s}, 1 \mathrm{H}), 8.20(\mathrm{~d}, J=7.8 \mathrm{~Hz}, 2 \mathrm{H}), 7.62(\mathrm{~d}$, $J=8.3 \mathrm{~Hz}, 1 \mathrm{H}), 7.53-7.44(\mathrm{~m}, 2 \mathrm{H}), 7.37(\mathrm{t}, J=8.1 \mathrm{~Hz}, 1 \mathrm{H}), 7.31-7.24(\mathrm{~m}, 2 \mathrm{H}) ;{ }^{13} \mathrm{C}$ NMR $(100$ MHz, DMSO- $\left.d_{6}\right): \delta 160.1,140.9,138.2,137.9,129.4,124.6,124.2,122.3,121.7,121.2,120.9$, $116.2,112.2,111.9,106.6$. 


\section{Radical trapping experiment:}

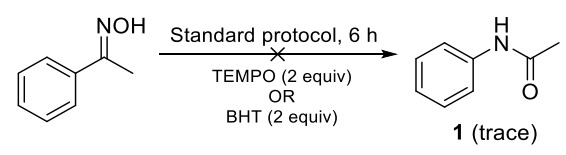

In a round bottom flask equipped with a stirring bar and water condenser, the solution of acetophenone oxime (50 mg, 1 equiv, $0.37 \mathrm{mmol}$ ), ammonium persulfate (168 mg, 2 equiv, 0.74 mmol), TEMPO (115 mg, 2 equiv, $0.74 \mathrm{mmol})$ and DMSO (157 $\mu \mathrm{L}, 6$ equiv, $2.46 \mathrm{mmol})$ in 1,4dioxane $(2 \mathrm{~mL})$ was heated at $100{ }^{\circ} \mathrm{C}$ for $6 \mathrm{~h}$. The reaction was followed by TLC, however only a trace amount of amide $\mathbf{1}$ was observed.

The same reaction was performed in the presence of BHT (326 mg, 4 equiv, $1.47 \mathrm{mmol}$ ) as a radical scavenger. In this case also only a trace amount of product $\mathbf{1}$ was seen on TLC.

Trapping of intermediates with BHT following the above mentioned procedure:

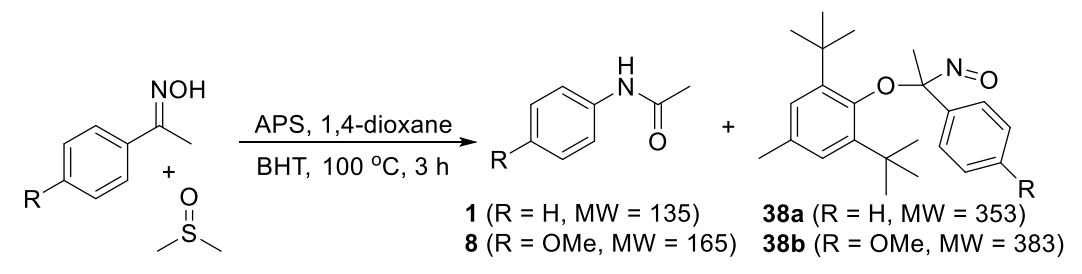

The trapped intermediates 38a-b were detected by LC-MS and HRMS. 


\section{6. ${ }^{18}$ O Labeling Experiment:}

\section{${ }^{18} \mathrm{O}$-enriched acetophenone oxime to ${ }^{18} \mathrm{O}$-enriched acetanilide (40):}

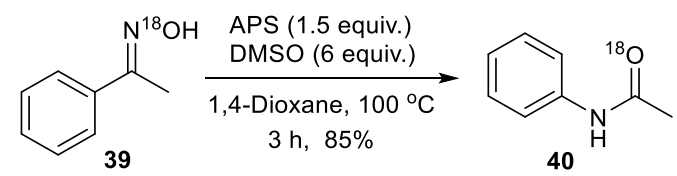

${ }^{18} \mathrm{O}$-Labelled $\mathrm{NH}_{2}{ }^{18} \mathrm{OH}$ was prepared starting from $\mathrm{H}_{2}{ }^{18} \mathrm{O}$ and ammonia following the reported procedure. ${ }^{39}$ It was used for the preparation of previously known ${ }^{18} \mathrm{O}$-labelled acetophenone oxime $39 .{ }^{39 b}$ Treatment of the oxime $\mathbf{3 9}$ under our standard protocol (General procedure, page 1) furnished ${ }^{18} \mathrm{O}$-labelled acetanilide (40) in $85 \%$ yield. Spectral and analytical data was in agreement with the above reported compound $\mathbf{1}$. More than $50 \%$ of acetanilide (40) had incorporated the heavy oxygen atom (approximate calculation from Mass spectrum). HRMS-ESI $(\mathrm{m} / z)$ calcd $\left[\mathrm{C}_{8} \mathrm{H}_{9}{ }^{18} \mathrm{ON}+\mathrm{H}\right]^{+}:$138.0799, found 138.0797. 


\section{References:}

1) Pakulski, M. M.; Mahato, S. K.; Bosiak, M. J.; Krzeminski, M. P.; Zaidlewicz, M. Tetrahedron Asymm. 2012, 23, 716.

2) (a) Kamps, J. J. A. G.; Belle, R.; Mecinović J. Org. Biomol. Chem. 2013, 11, 1103; b) Pusterla, I.; Bode, J. W. Angew. Chem. Int. Ed. 2012, 51, 513.

3) Mahajan, P. S.; Mahajan, J. P.; Mhaske, S. B. Synth. Commun. 2013, 43, 2508.

4) Liu, H.; Zhao, L.; Yuan, Y.; Xu, Z.; Chen, K.; Qiu, S.; Tan, H. ACS Catal. 2016, 6, 1732.

5) Hashimoto, T.; Hirose, D.; Taniguchi, T. Adv. Synth. \& Catal. 2015, 357, 3346.

6) Umanadh, Y.; Reddy, N. S.; Mukkanti, K.; Omprakash, G. Asian J. Chem. 2015, 27, 1209.

7) Chen, Y.; Liu, B.; Liu, X.; Yang, Y.; Ling, Y.; Jia, Y. Org. Process Res. Dev. 2014, 18, 1589.

8) Ho, S. S. H.; Go, M. L. Bioorg. Med. Chem. Lett. 2013, 23, 6127.

9) Gu, L.; Wang, W.; Liu, J.; Li, G.; Yuan, M. Green Chem. 2016, DOI: 10.1039/c6gc00402d.

10) Nimnual, P.; Tummatorn, J.; Thongsornkleeb, C.; Ruchirawat, S. J. Org. Chem. 2015, 80, 8657.

11) Noh, J.-H.; Kim, J. J. Org. Chem. 2015, 80, 11624.

12) Kumar, R.; Ponnada, S.; Enjamuri, N.; Pandey, J. K.; Chowdhury, B. Catal. Commun. 2016, 77,42 .

13) Palmer, B. D.; Rewcastle, G. W.; Thompson, A. M.; Boyd, M.; Showalter, H. D. H.; Sercel, A.D.; Fry, D. W.; Kraker, A. J.; Denny, W.A. J. Med. Chem. 1995, 38, 58.

14) Hayashi, K.; Choshi, T.; Chikaraishi, K.; Oda, A.; Yoshinaga, R.; Hatae, N.; Ishikura, M.; Hibino, S. Tetrahedron 2012, 68, 4274.

15) a) Kamps, J. J. A. G.; Belle, R.; Mecinović J. Org. Biomol. Chem. 2013, 11, 1103; b) Pusterla, I.; Bode, J. W. Angew. Chem. Int. Ed. 2012, 51, 513. 
Chloroform-d

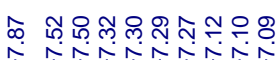

i

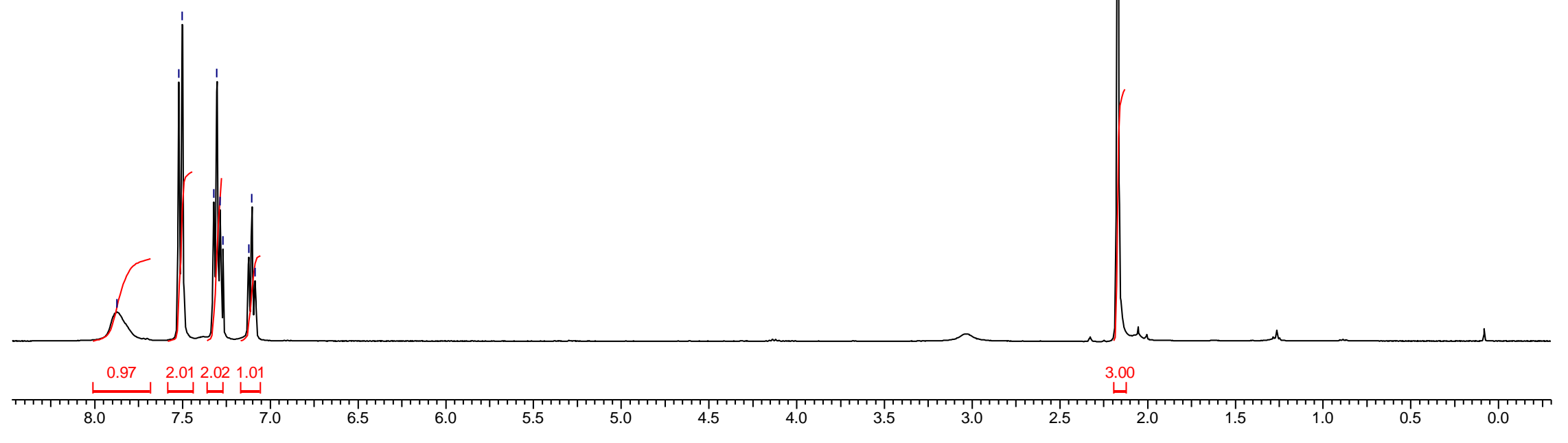




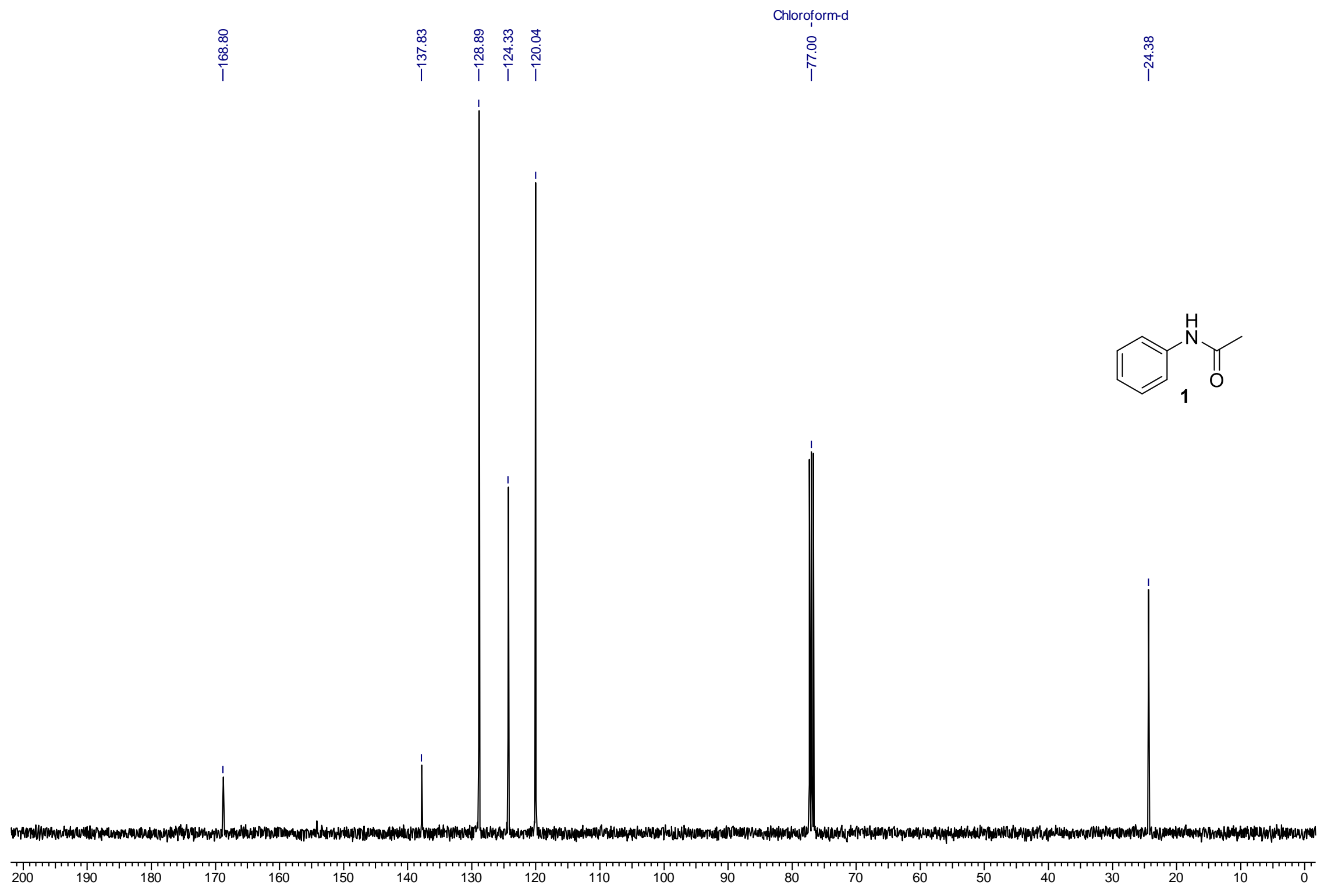




$$
\perp
$$


Chloroform-d

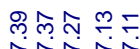

梌简

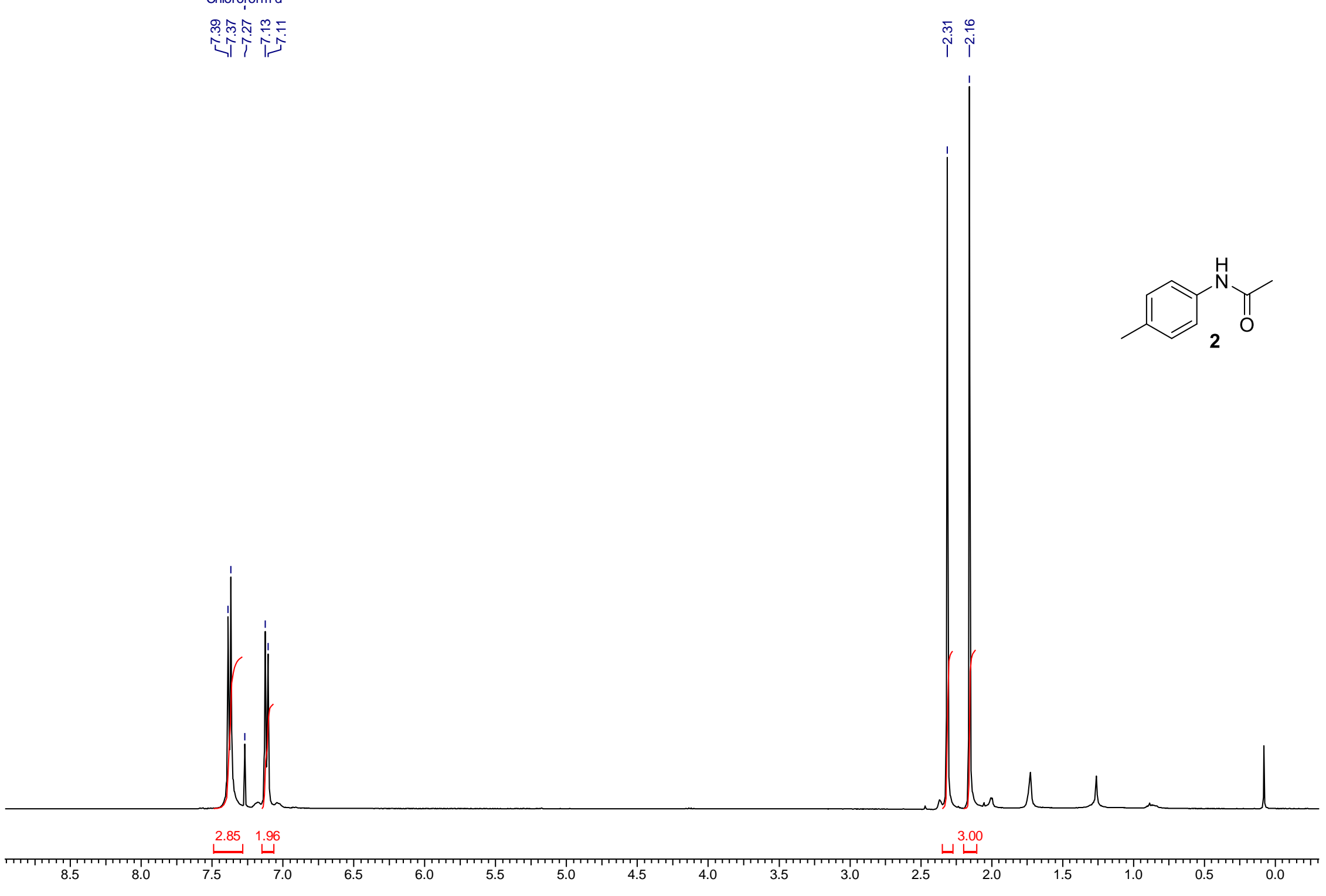




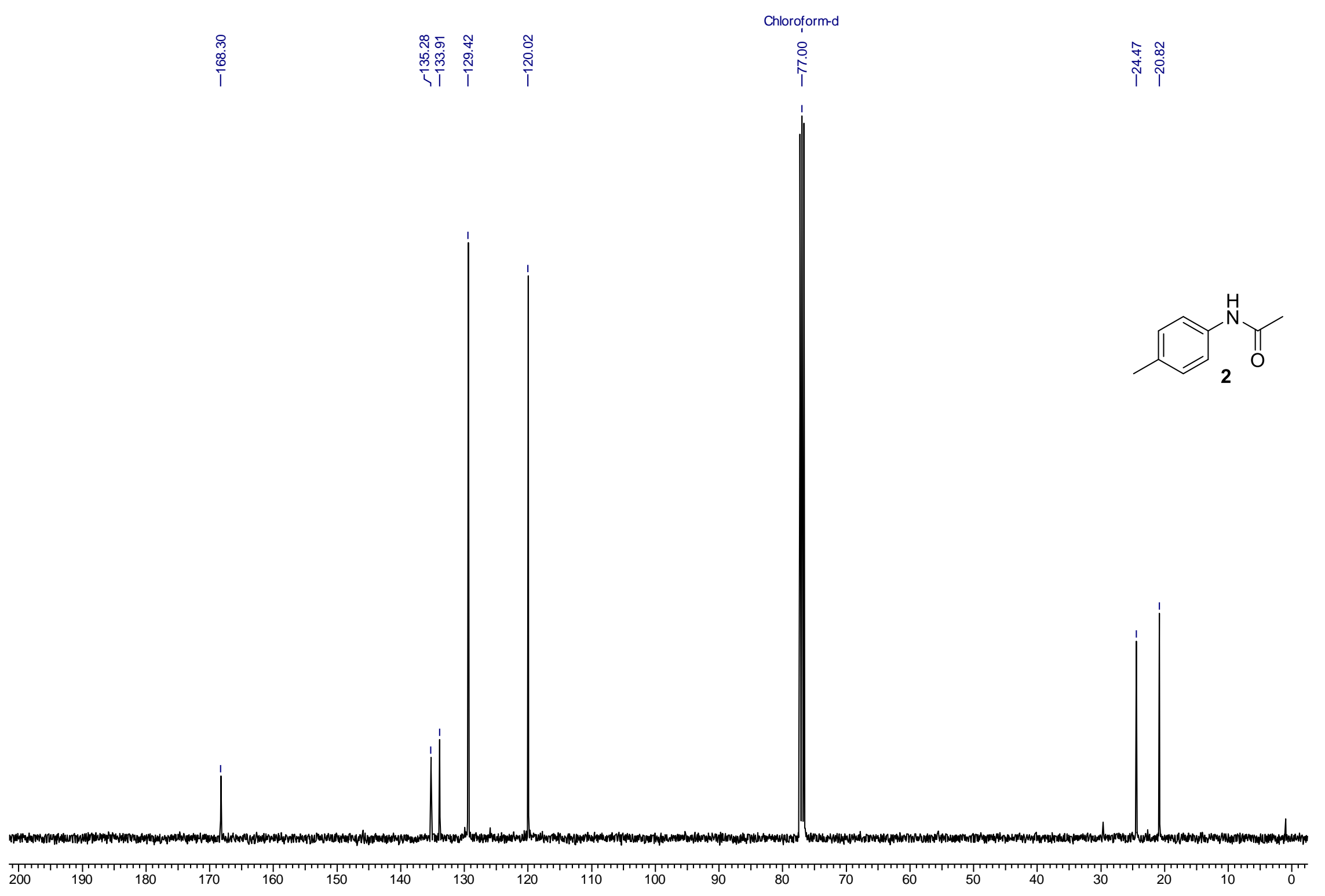


Chloroform-d

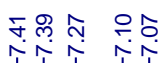

Ұ

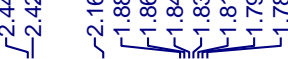

䠰

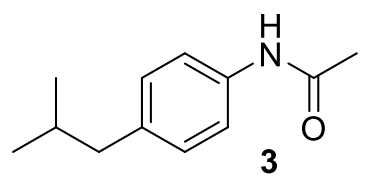

3

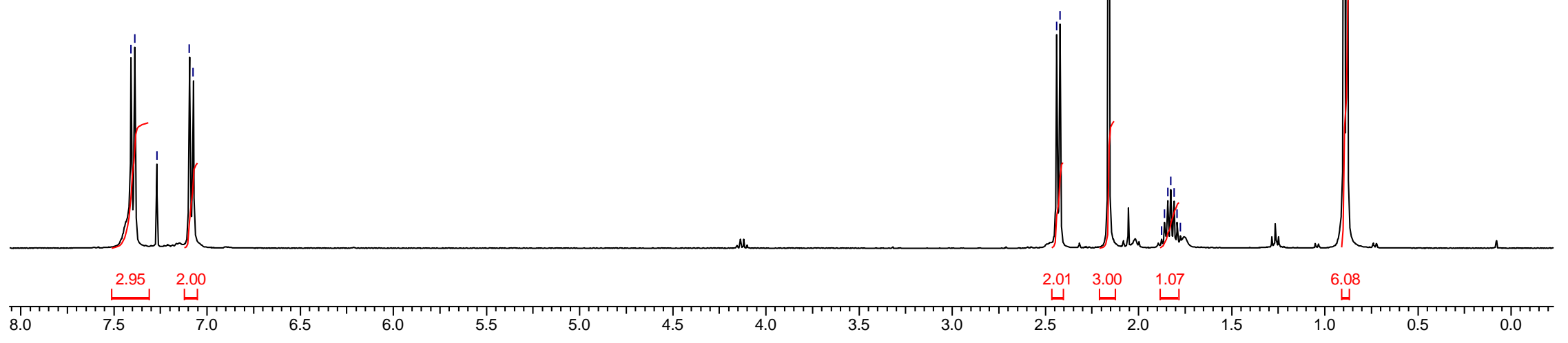


${ }^{13} \mathrm{C}$ NMR, 100MHz

\begin{tabular}{l} 
ले \\
00 \\
0 \\
0 \\
\hline
\end{tabular}

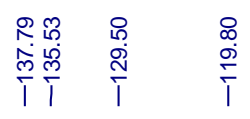

Chloroform-d

$\stackrel{8}{i}$

$\stackrel{\substack{j \\ \stackrel{4}{j}}}{\stackrel{\infty}{\mathfrak{l}}}$

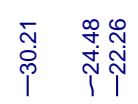

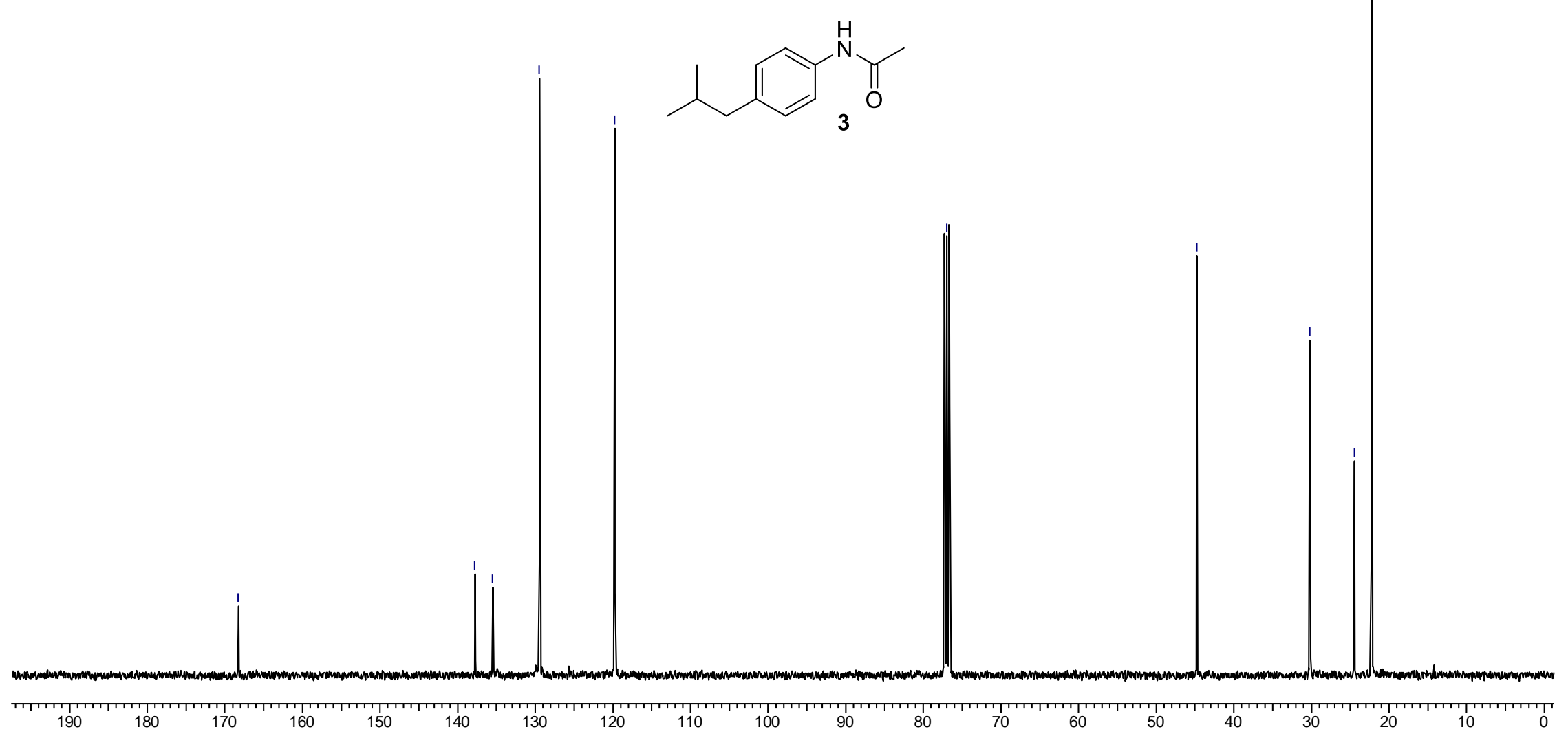

28 


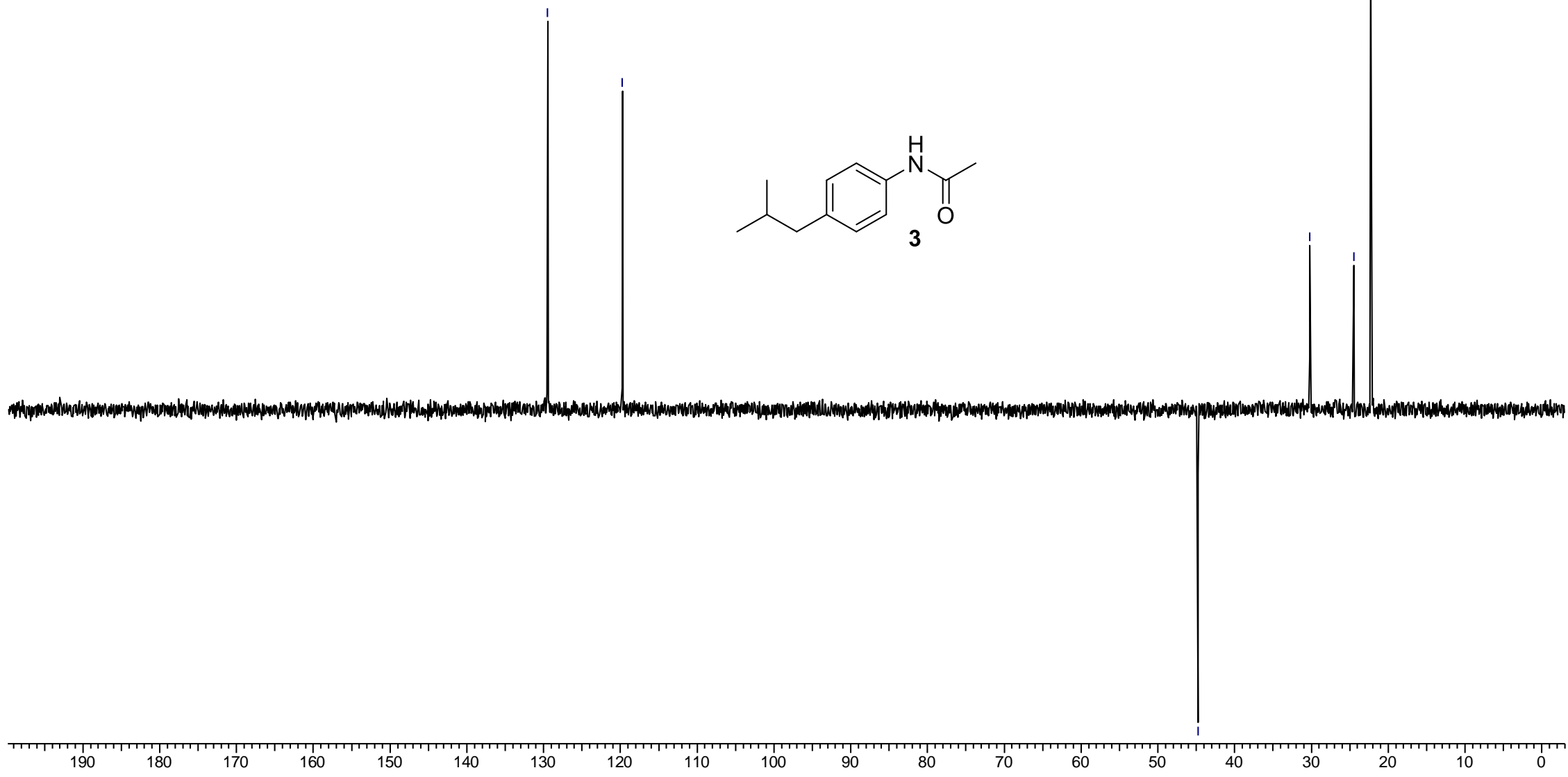


Chloroform-d

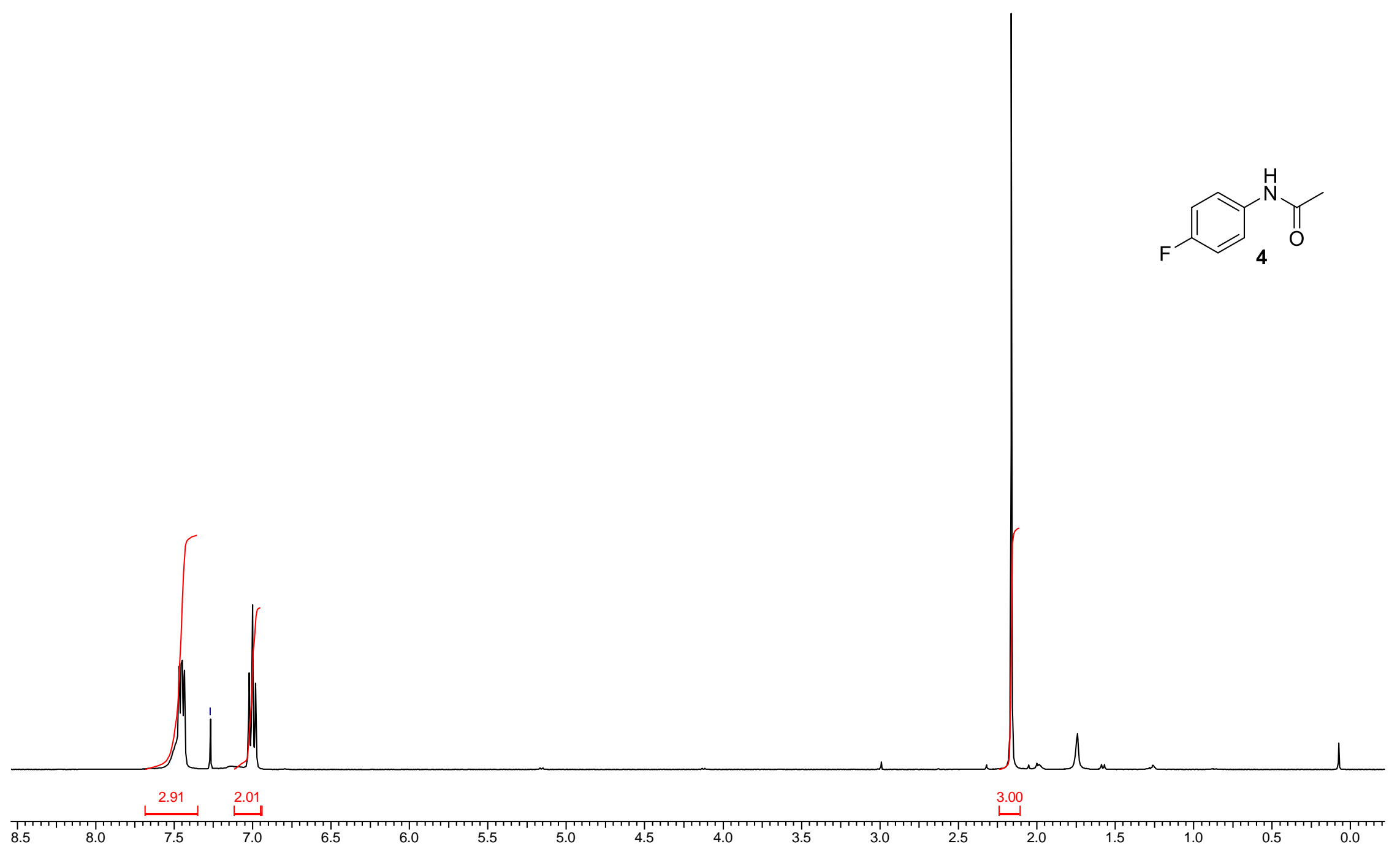




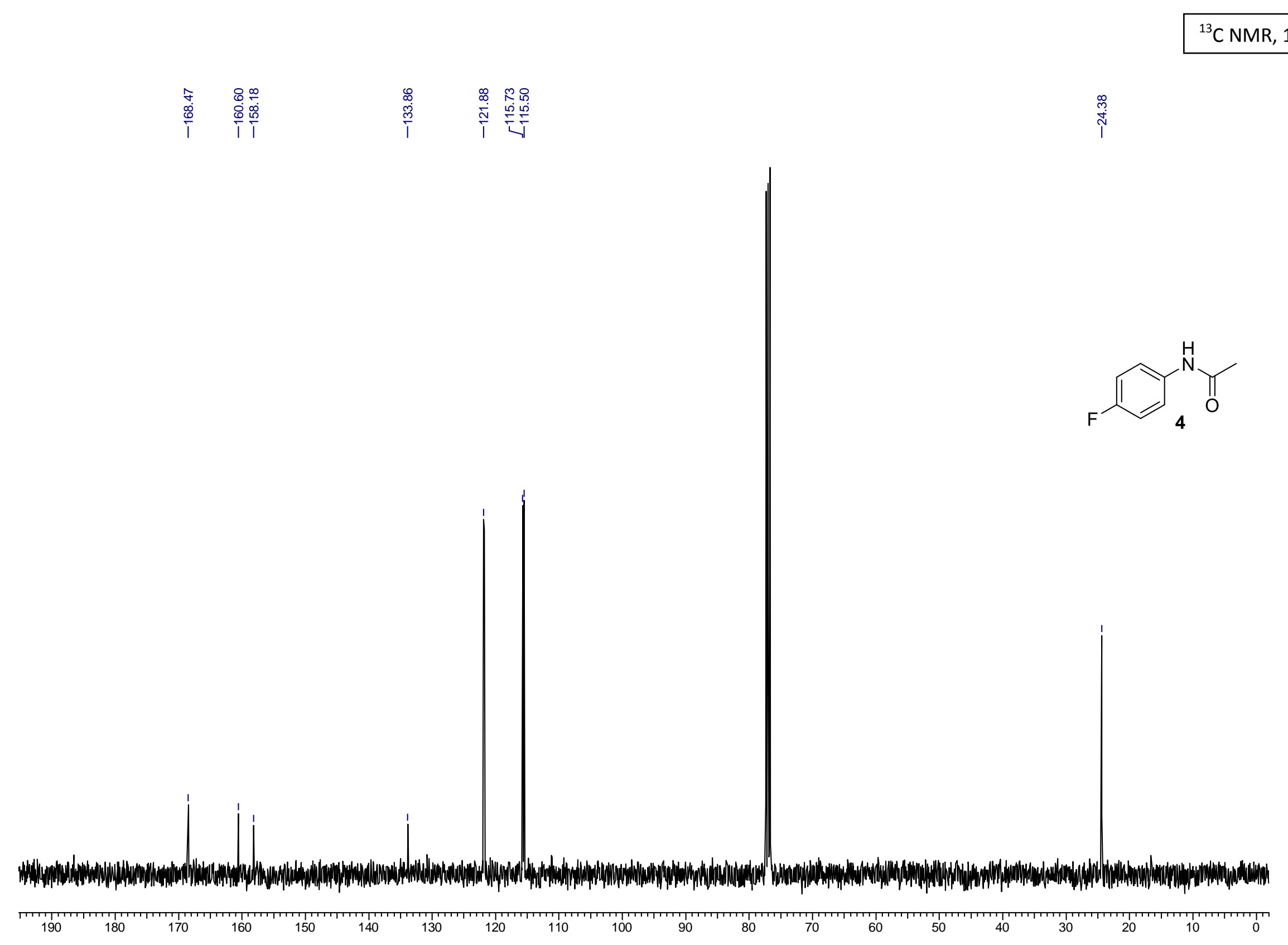




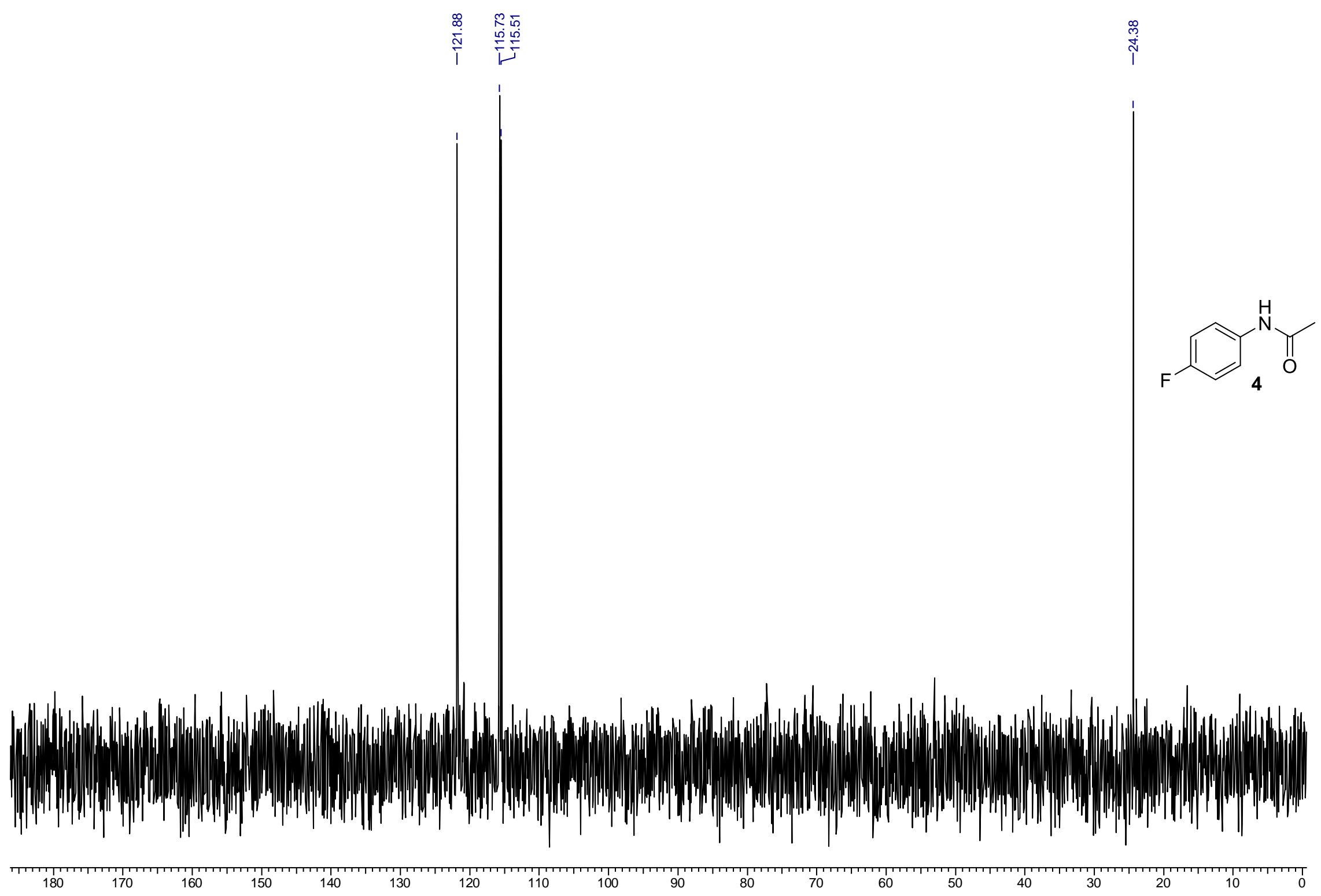




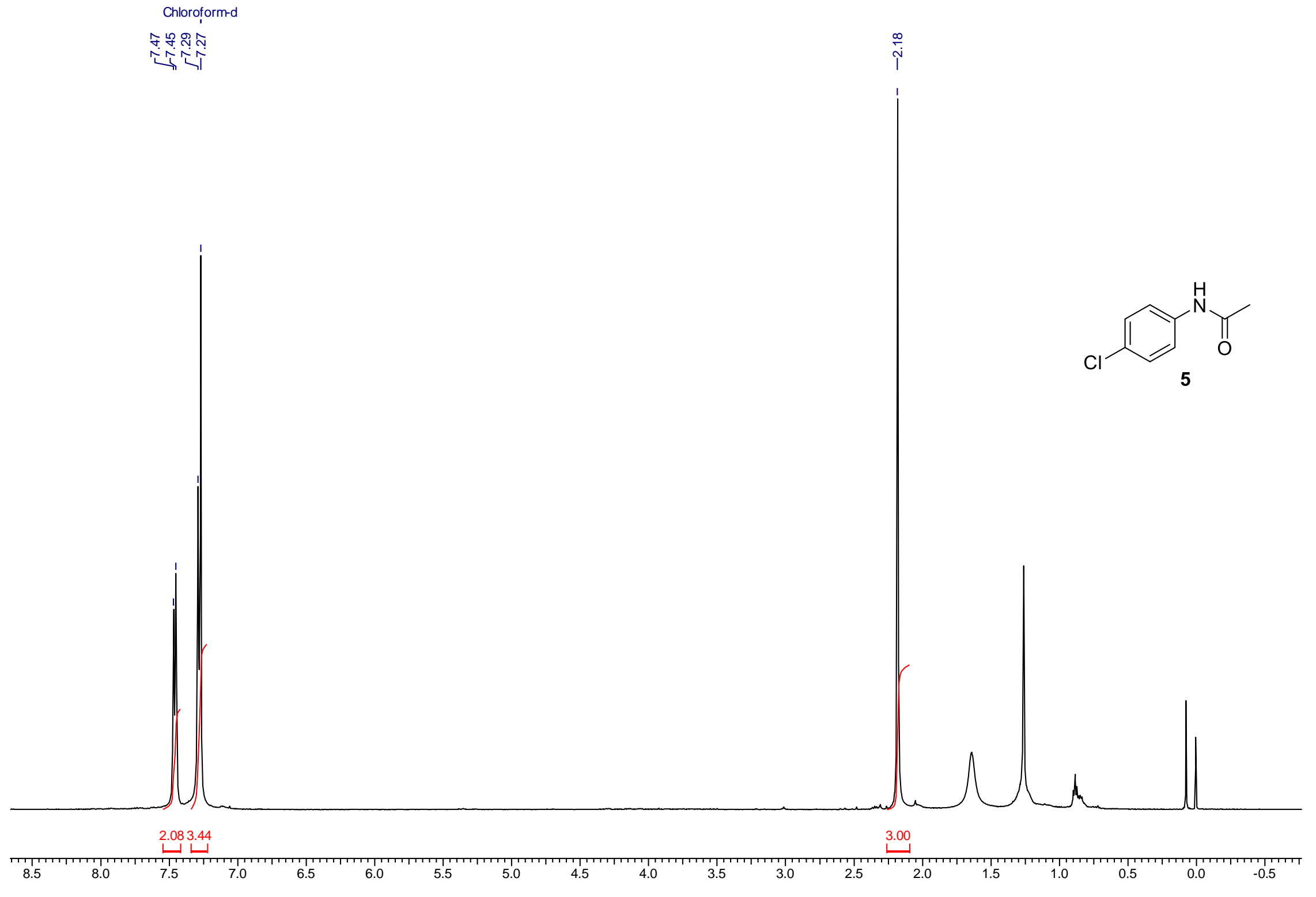




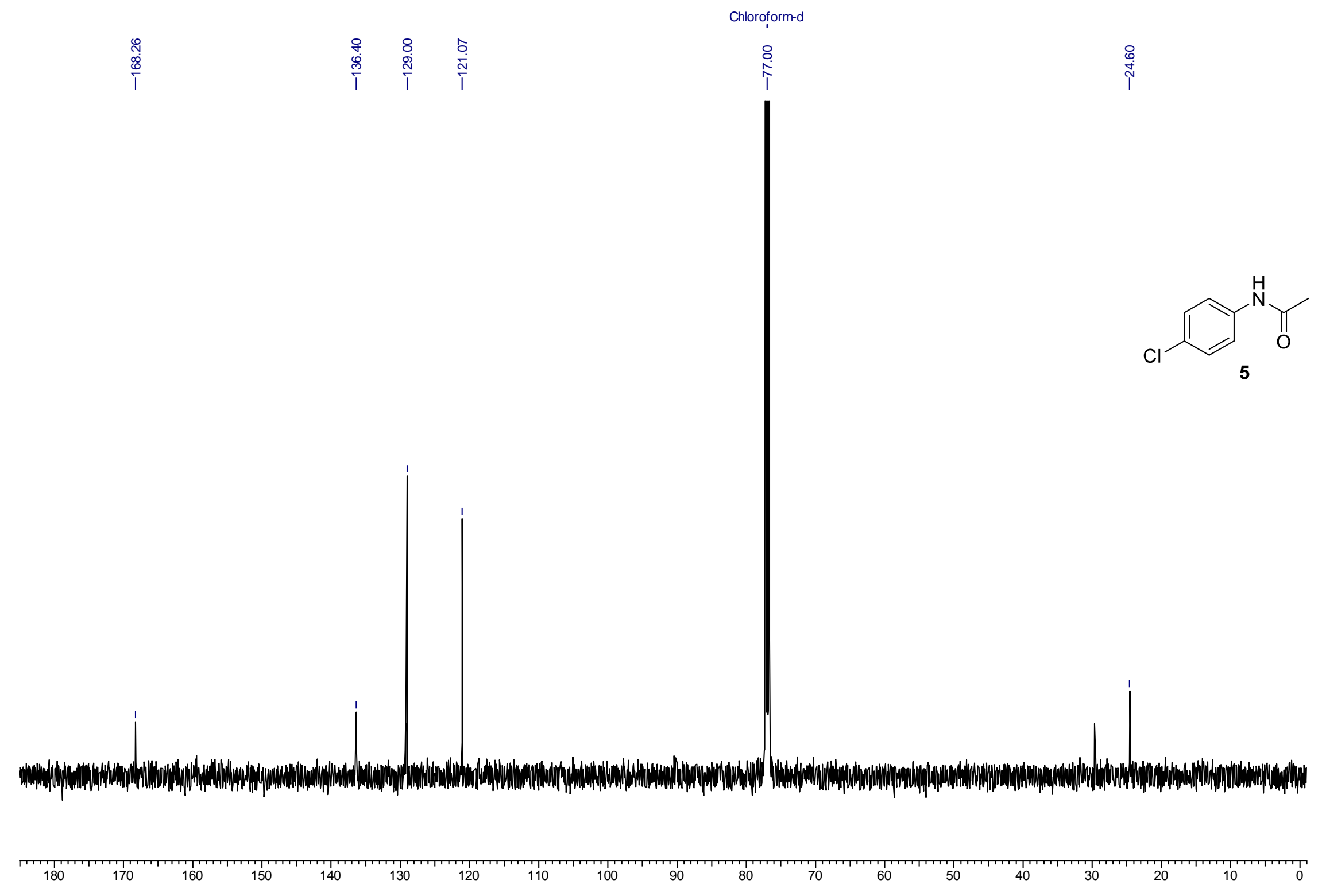




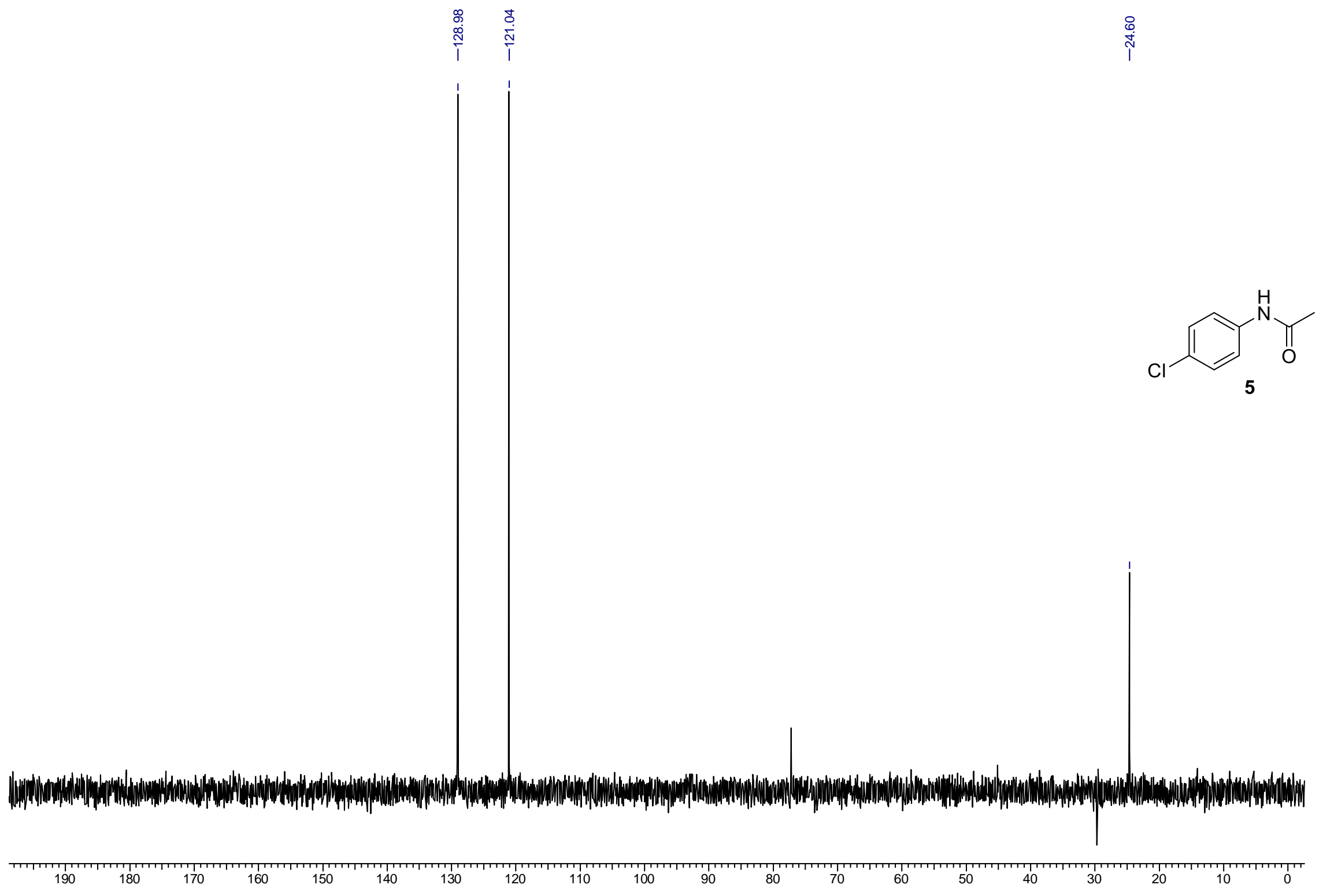




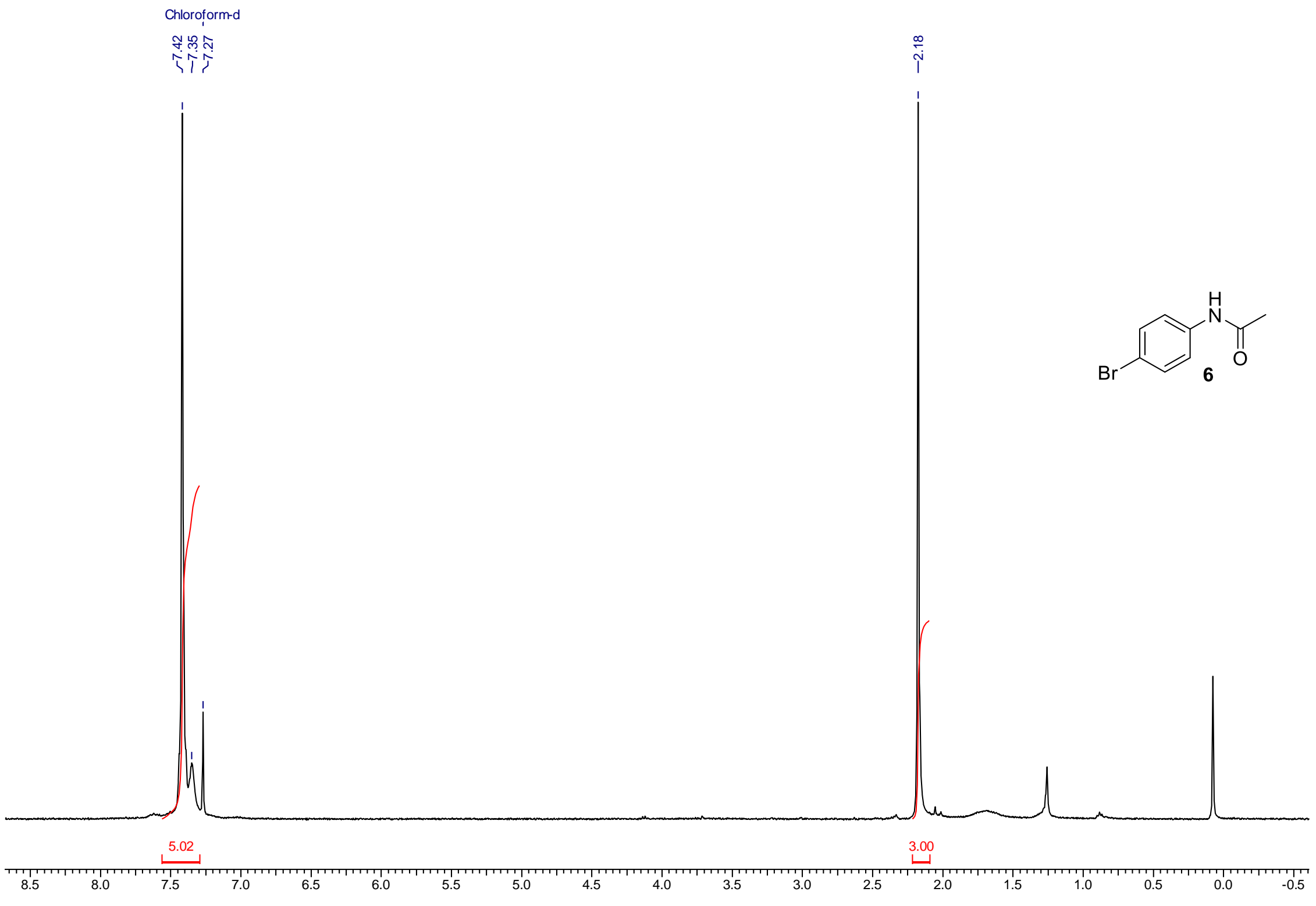




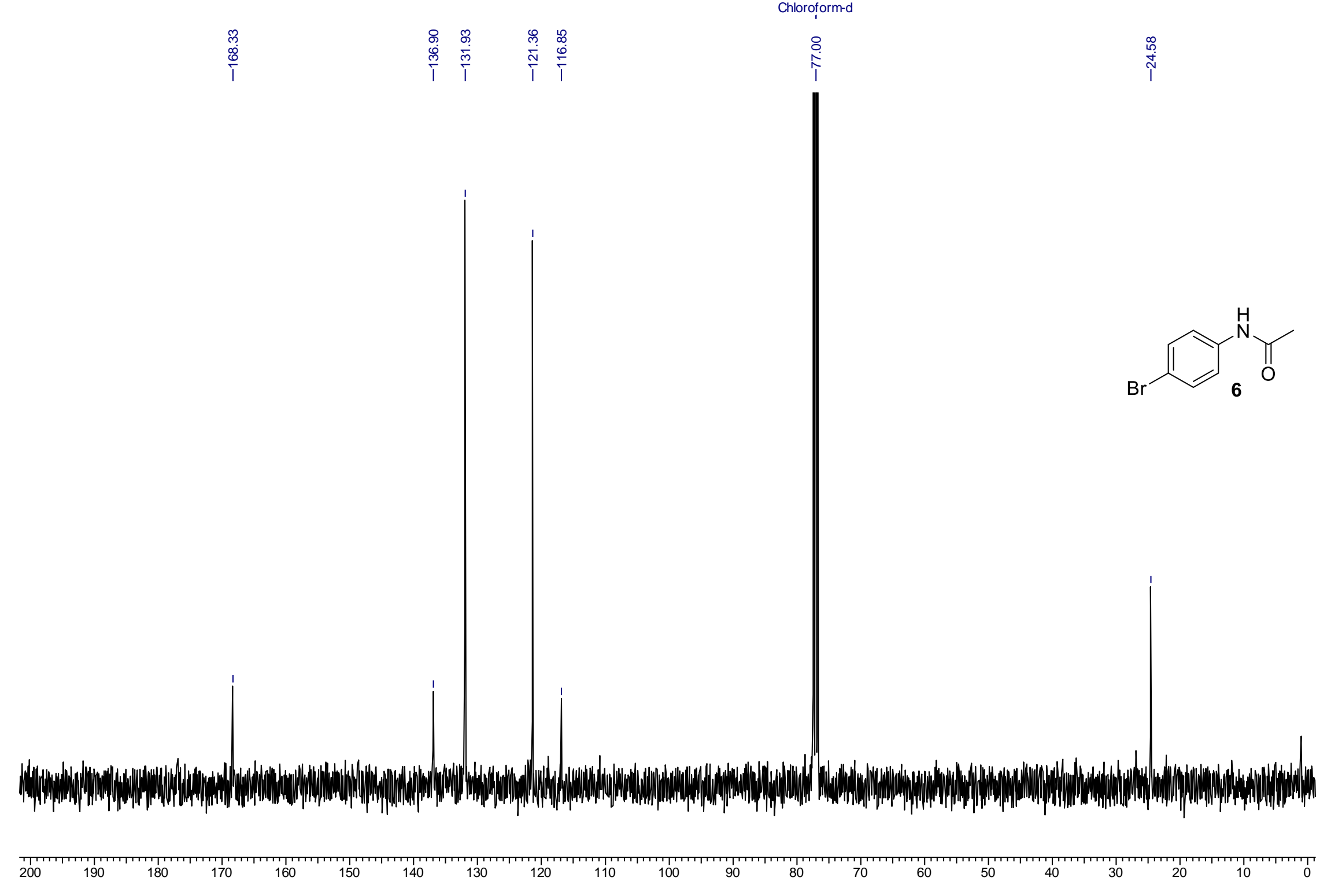




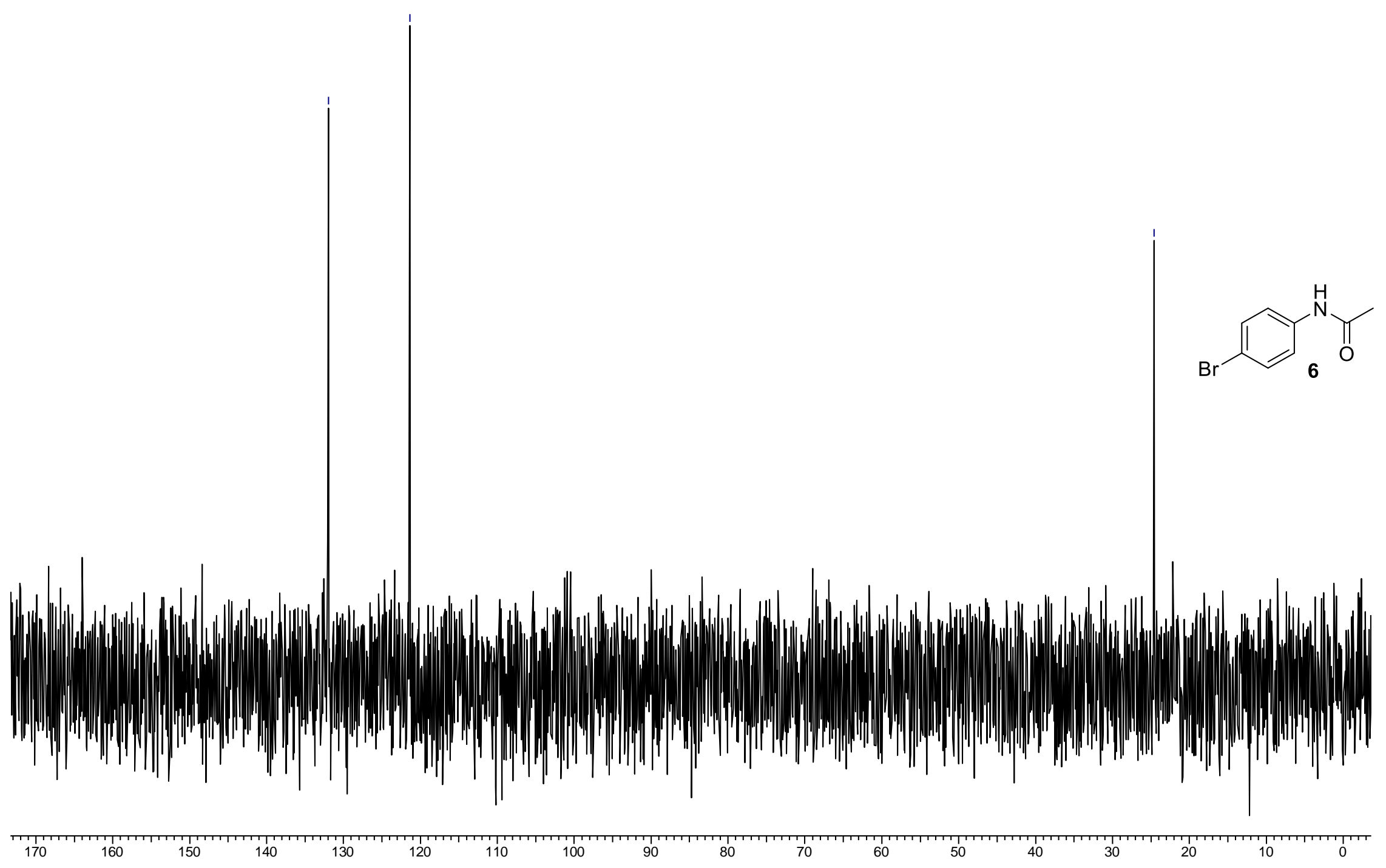


Chloroform-d

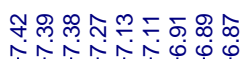

武分开

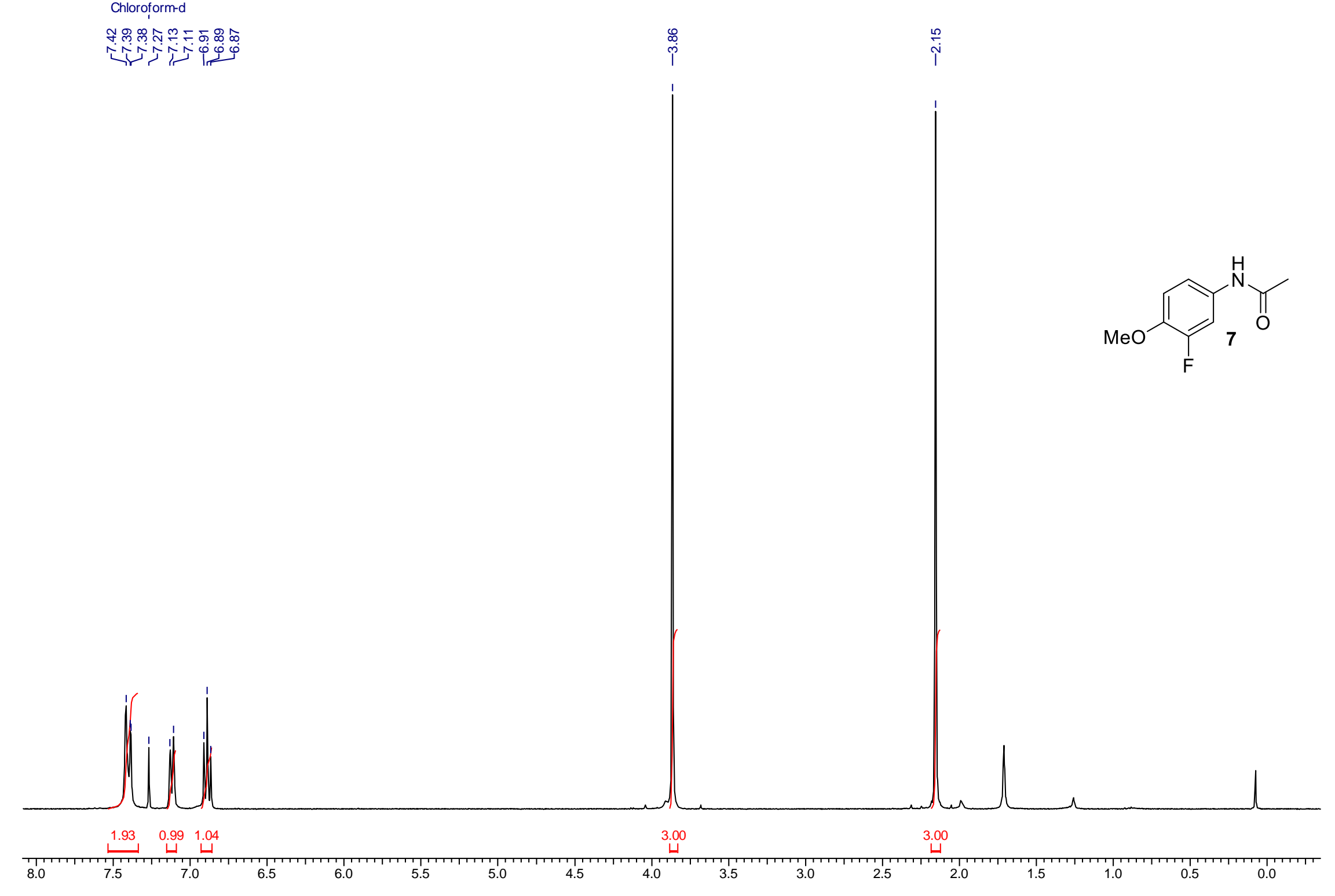




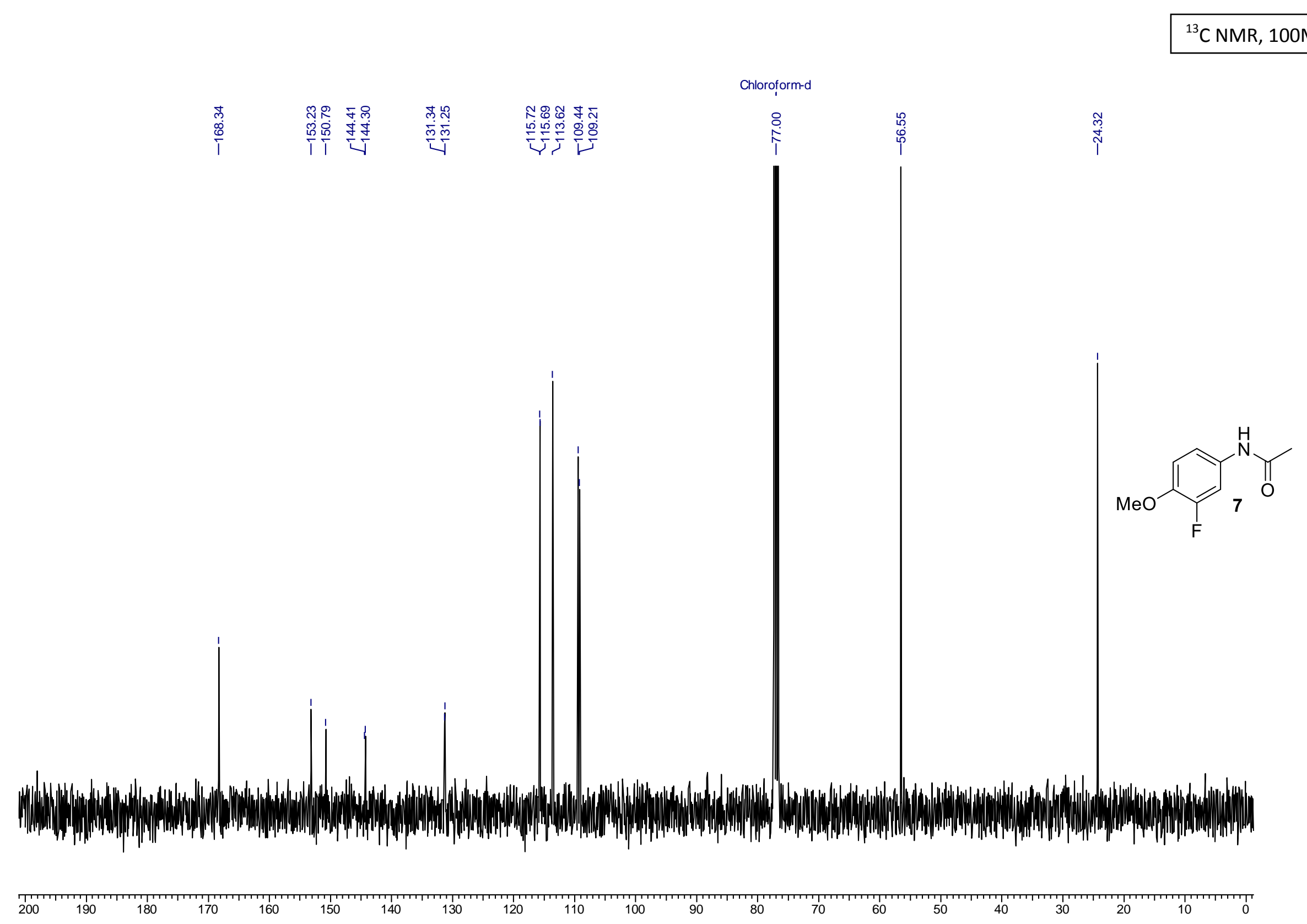




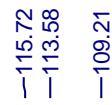
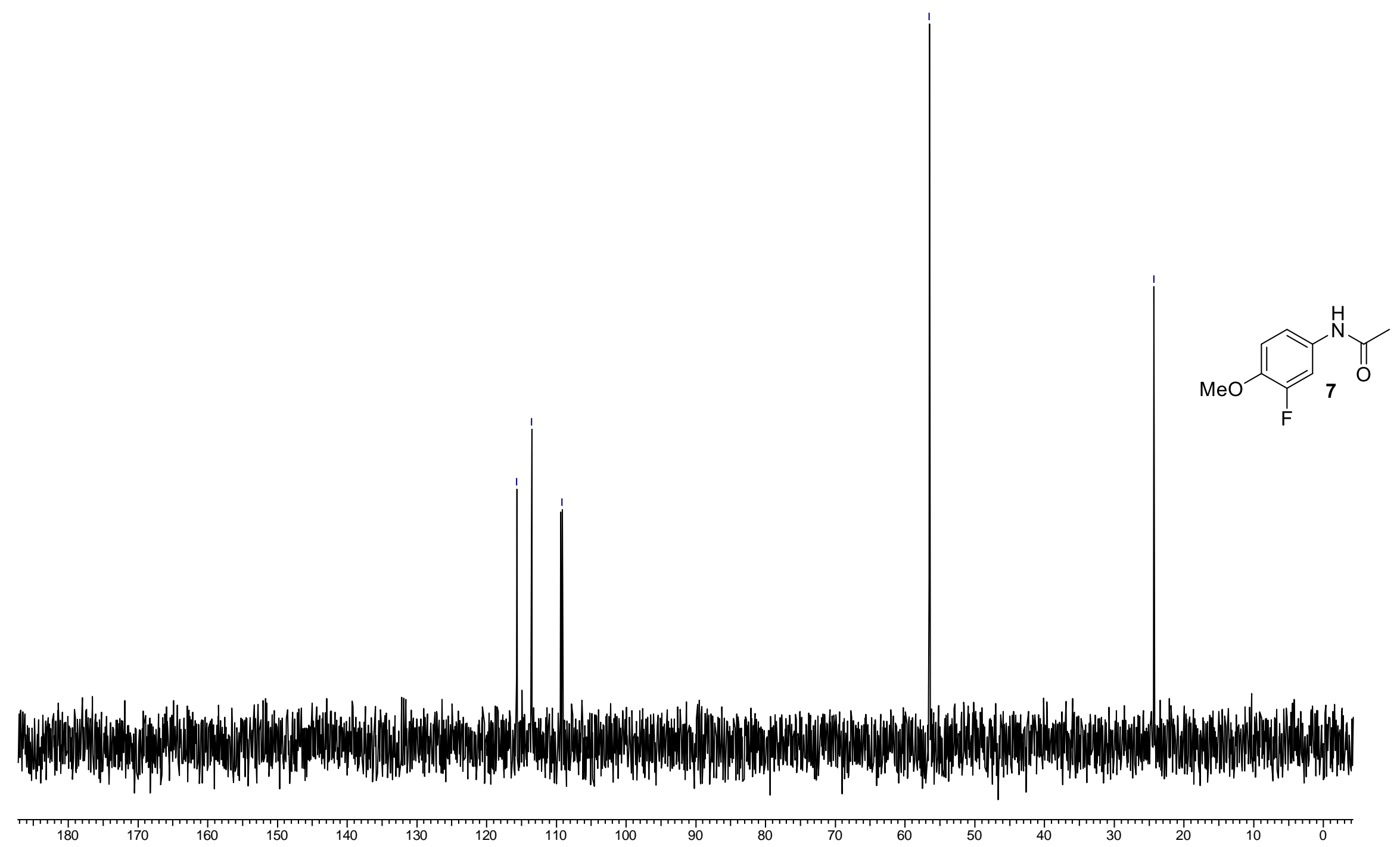
Chloroform-d

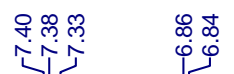

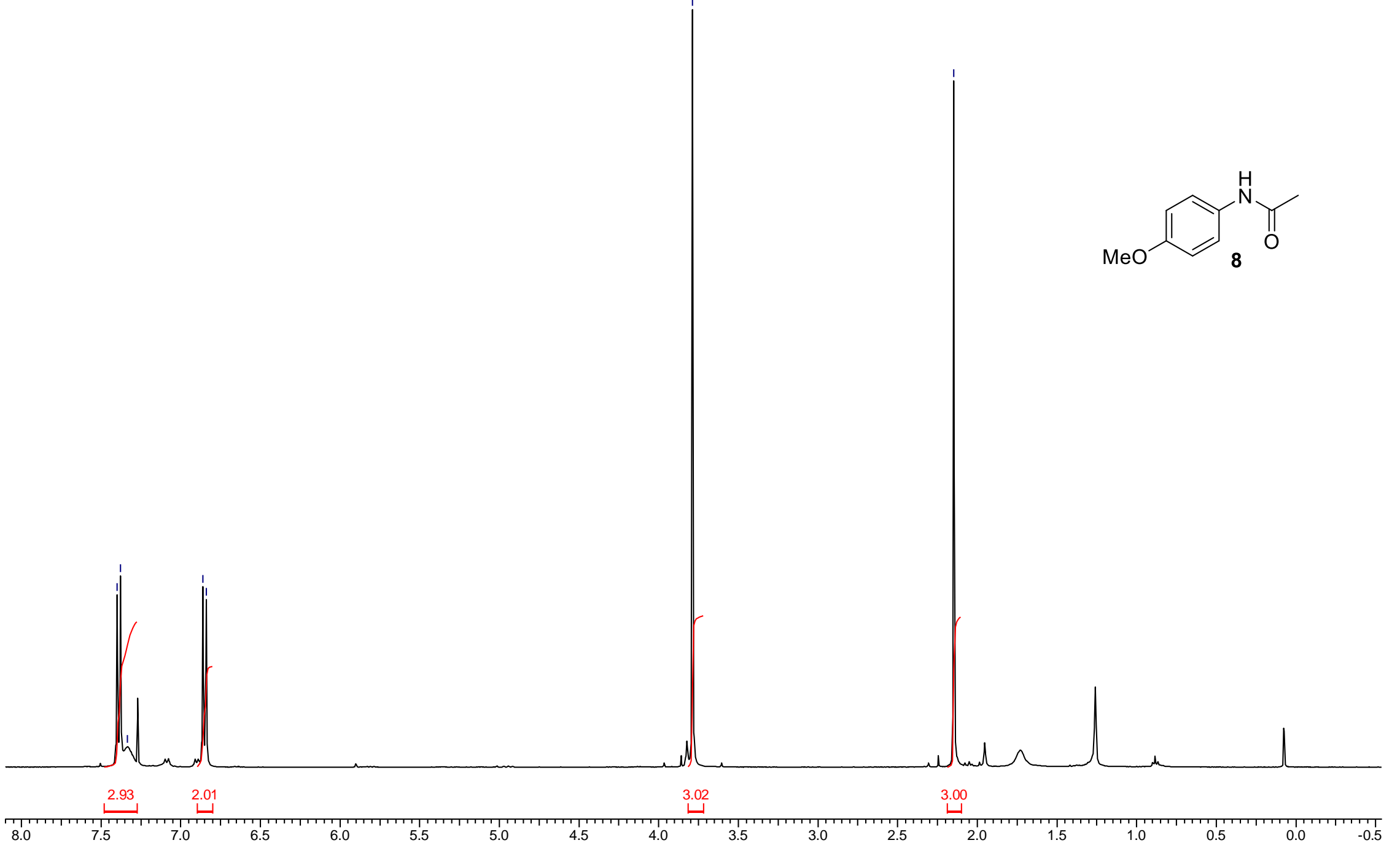


${ }^{13} \mathrm{C}$ NMR, $100 \mathrm{MHz}$

\begin{tabular}{l}
0 \\
0 \\
0 \\
0 \\
\hline
\end{tabular}

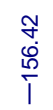

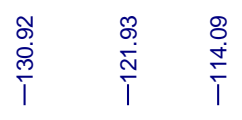

量

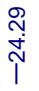

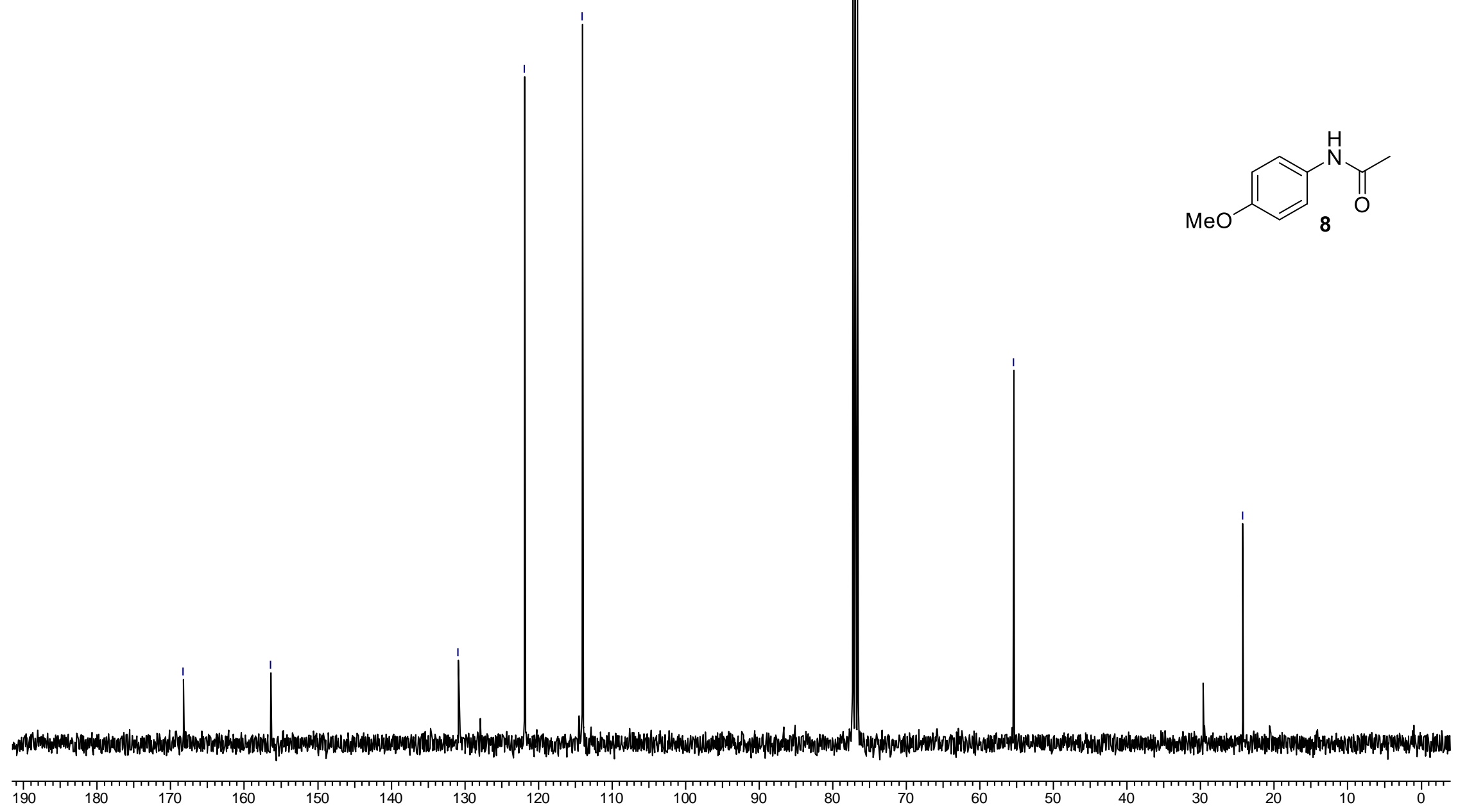

43 


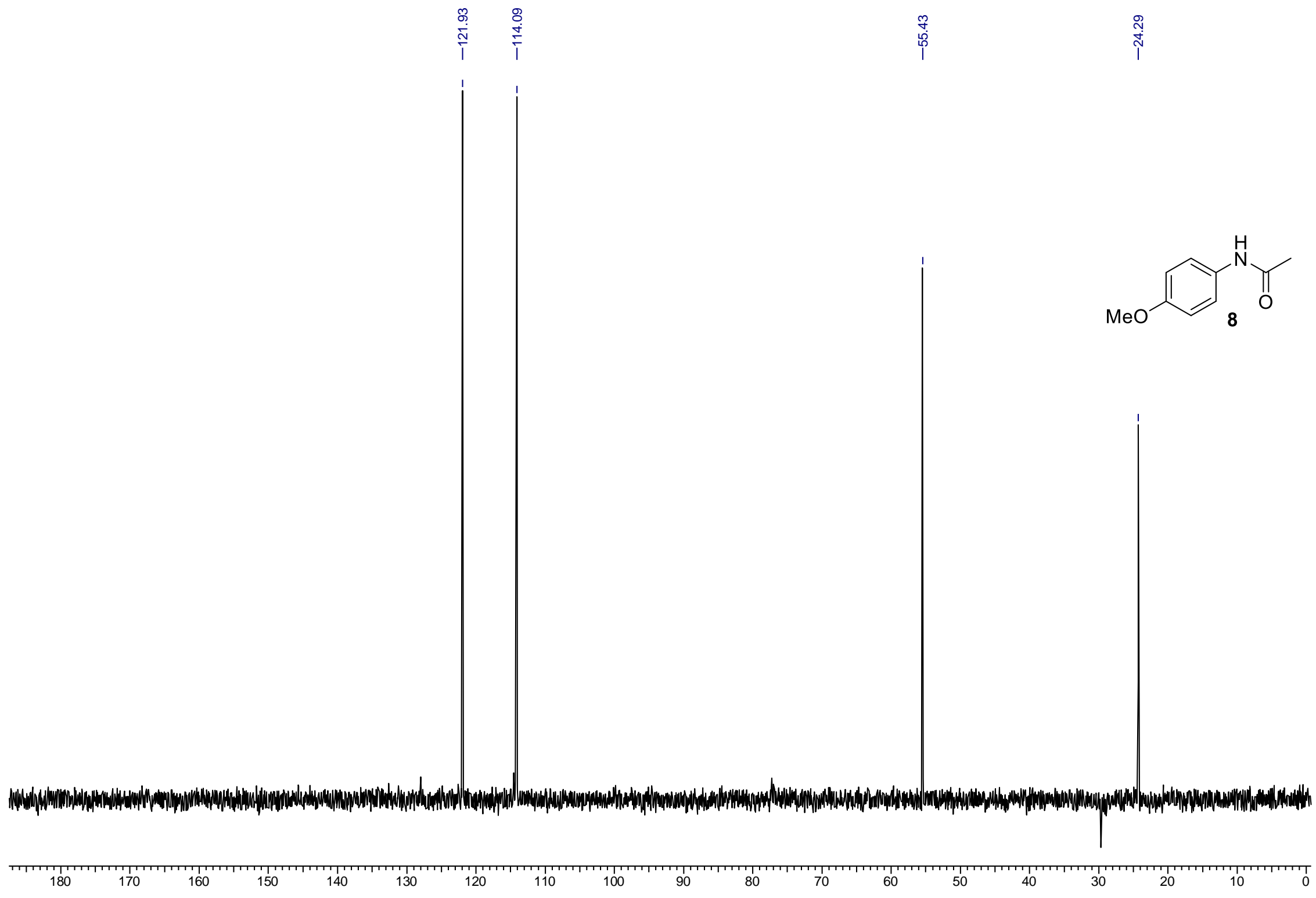




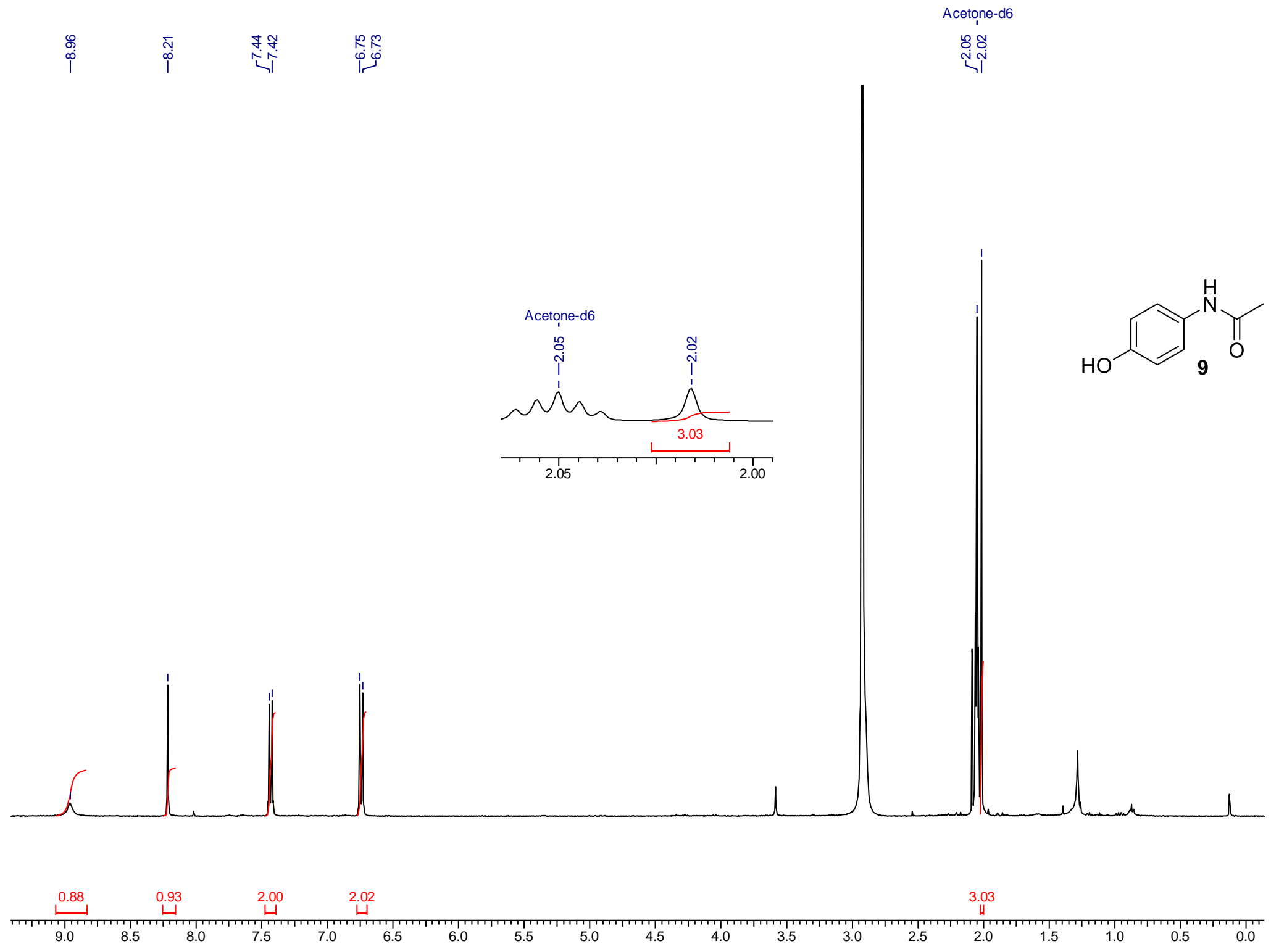




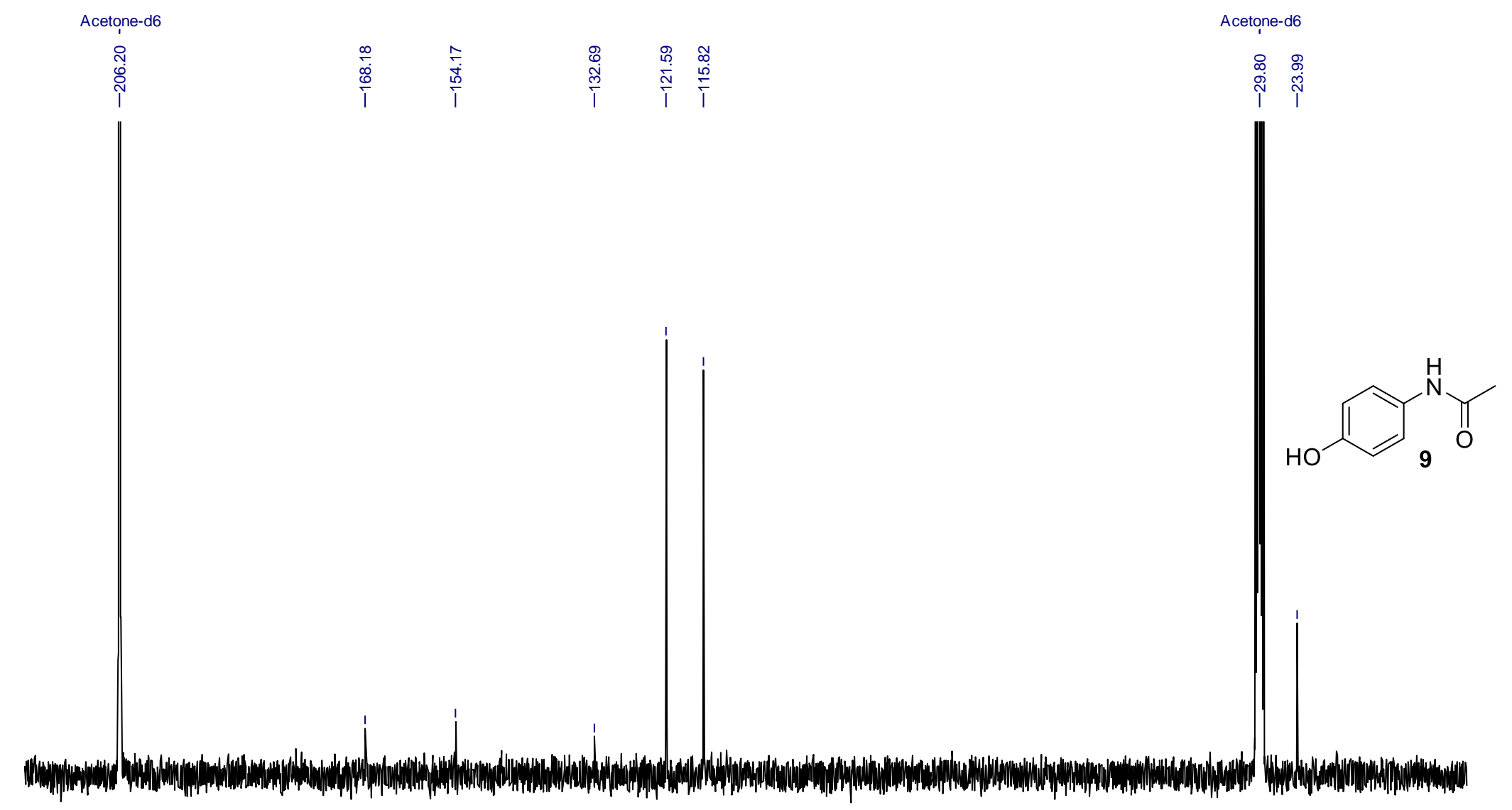

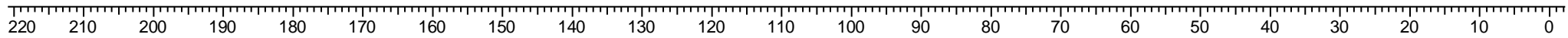




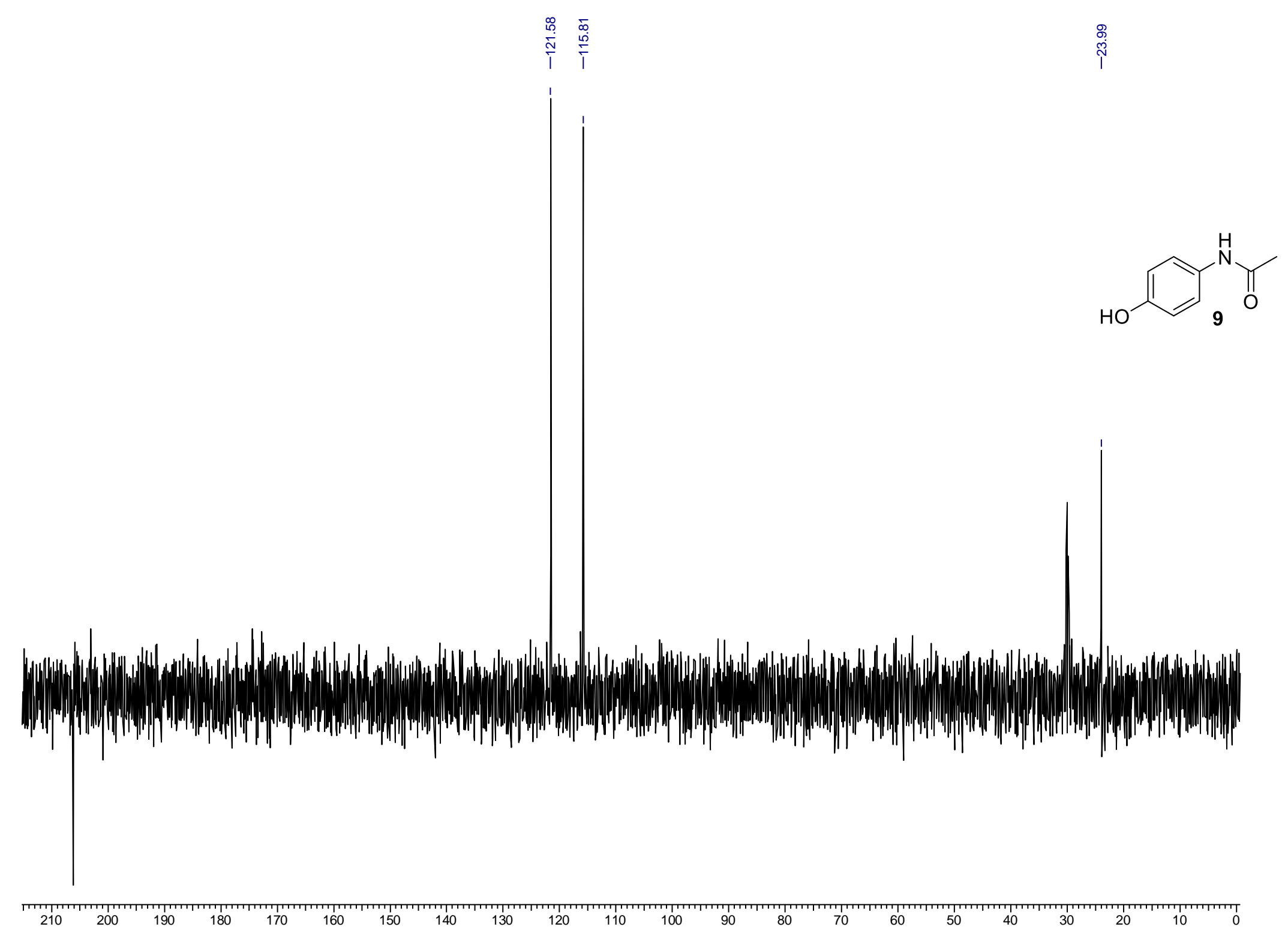




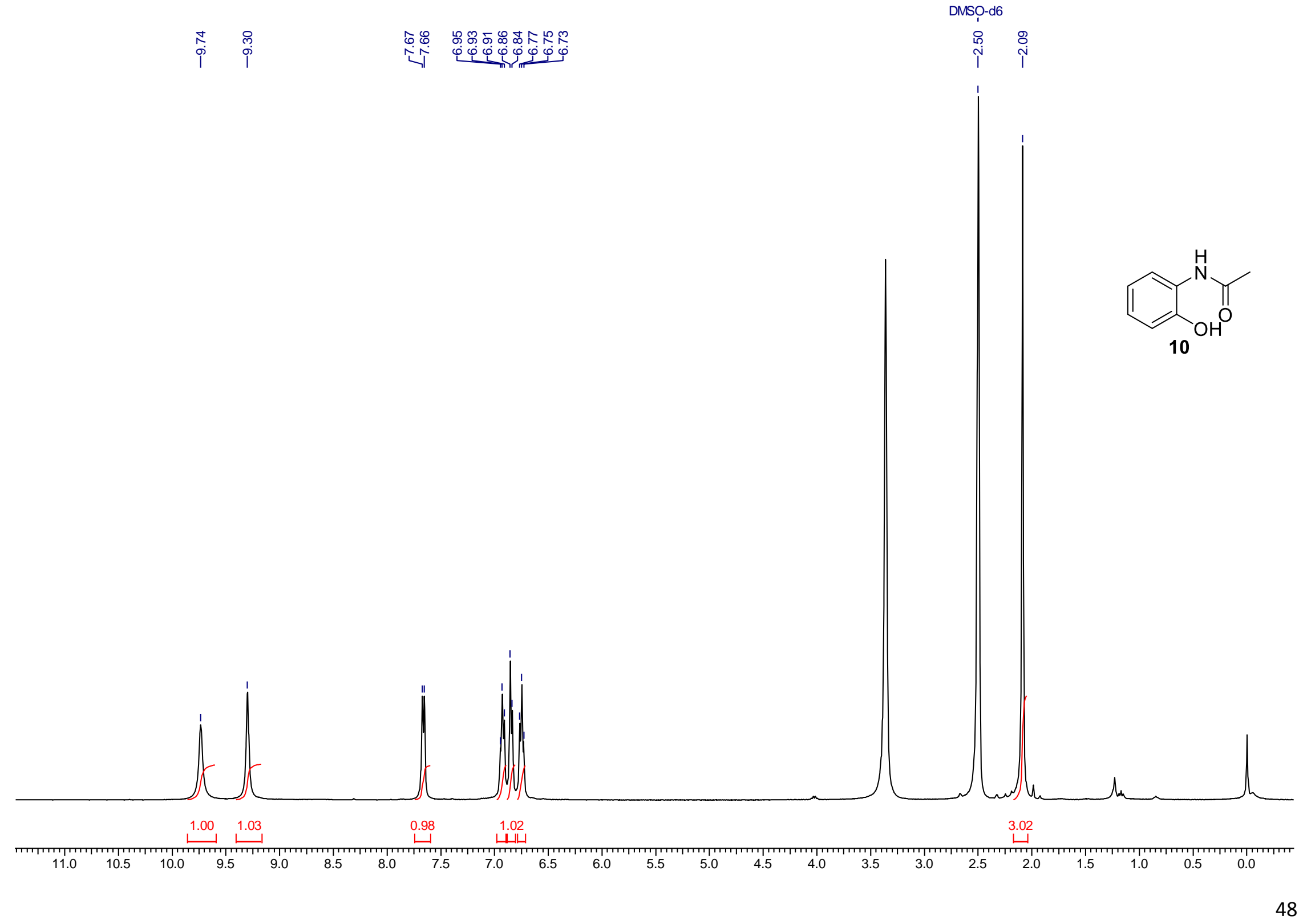




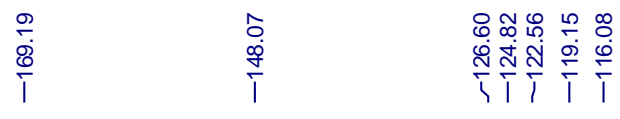

DMSO-d6
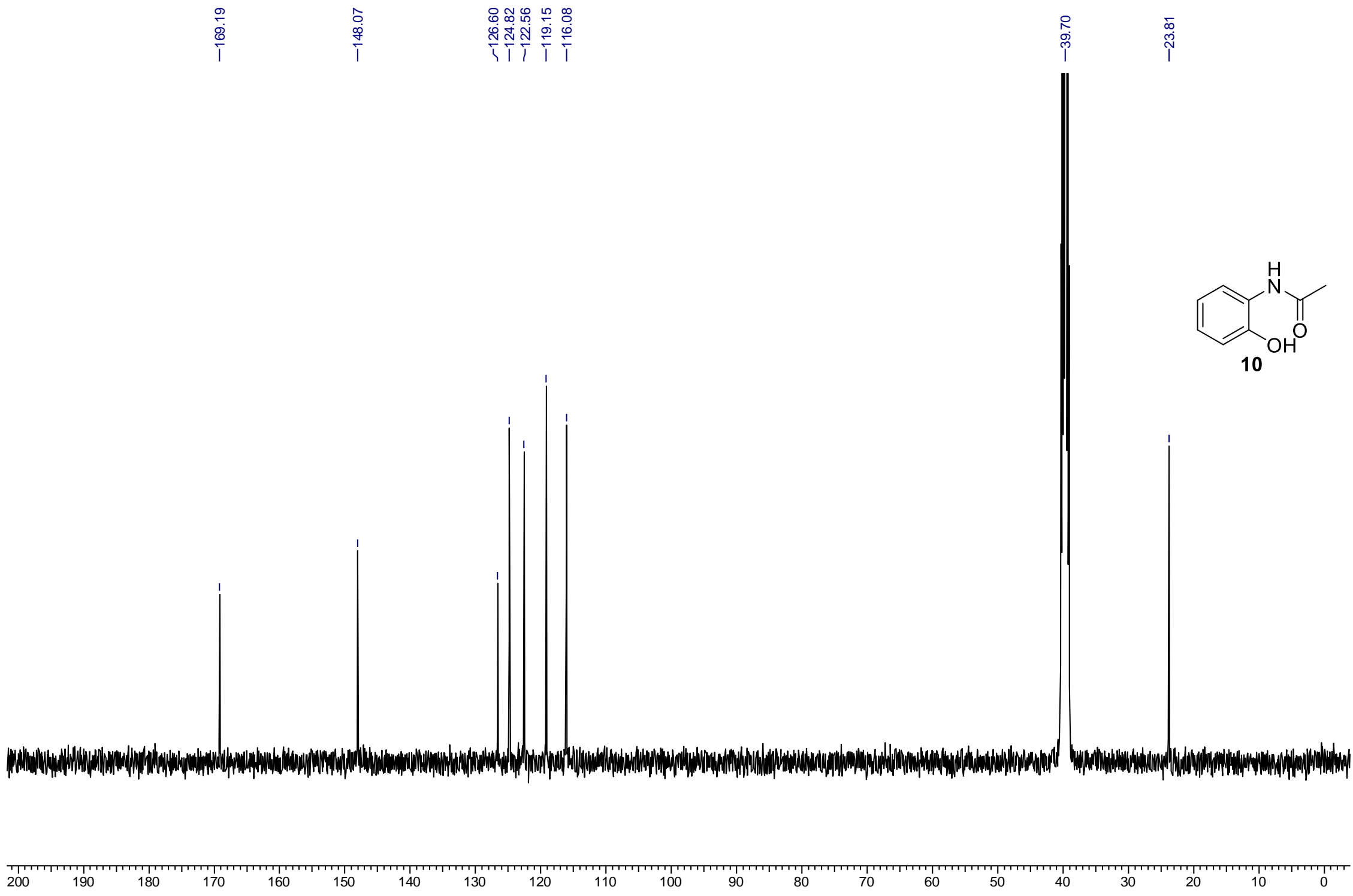


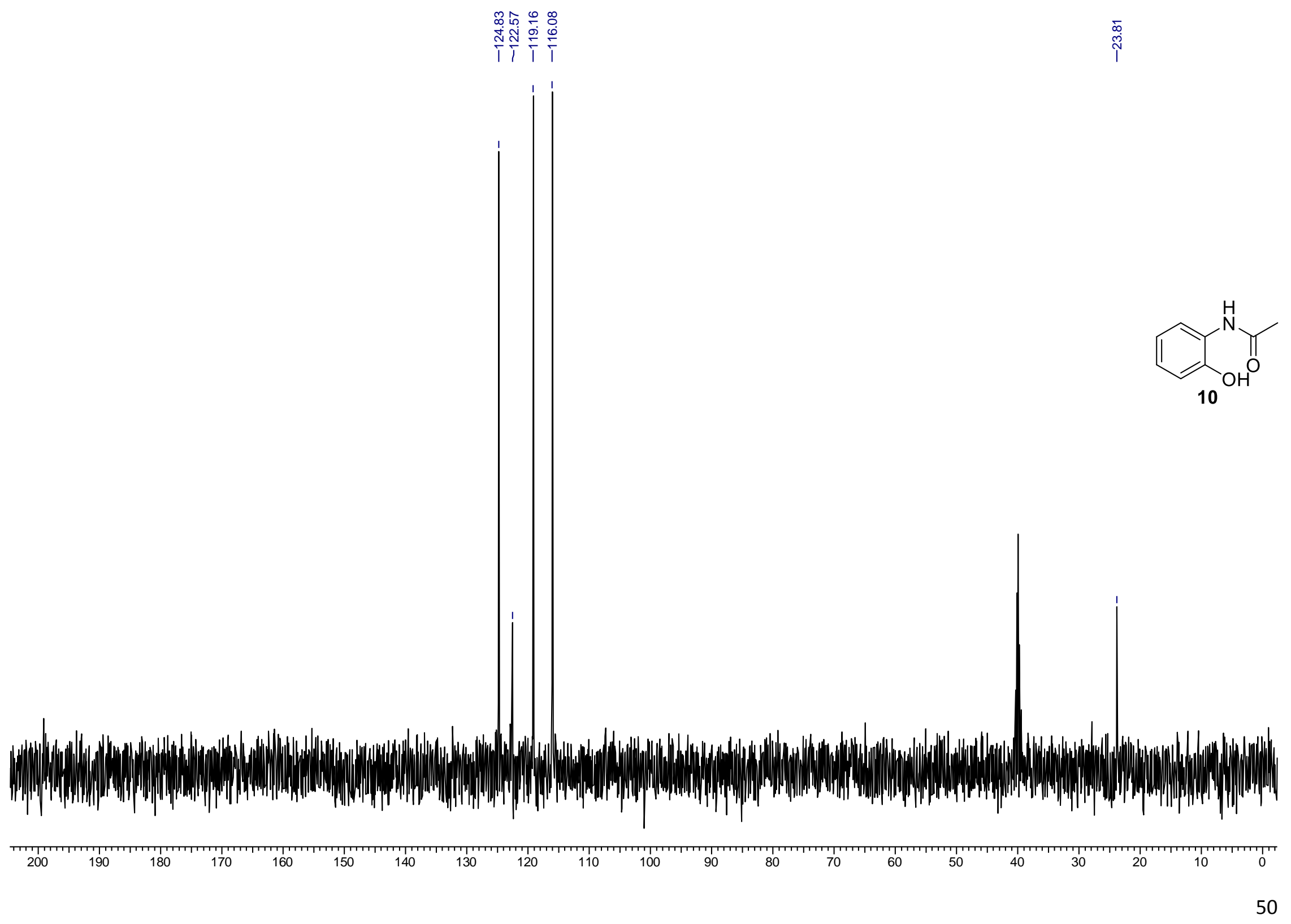


Chloroform-d

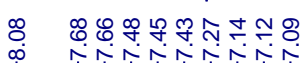

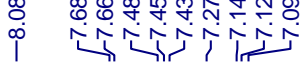

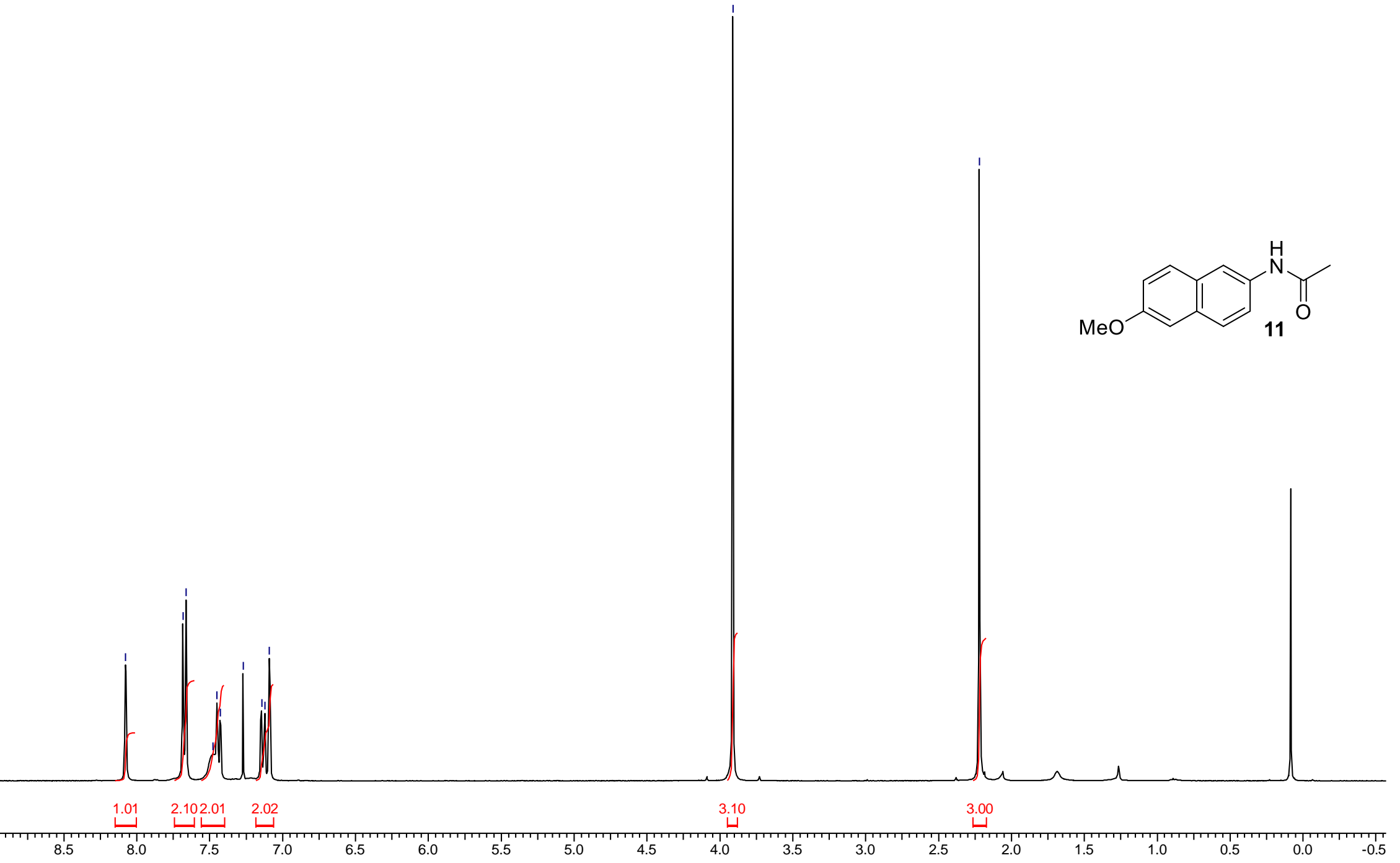




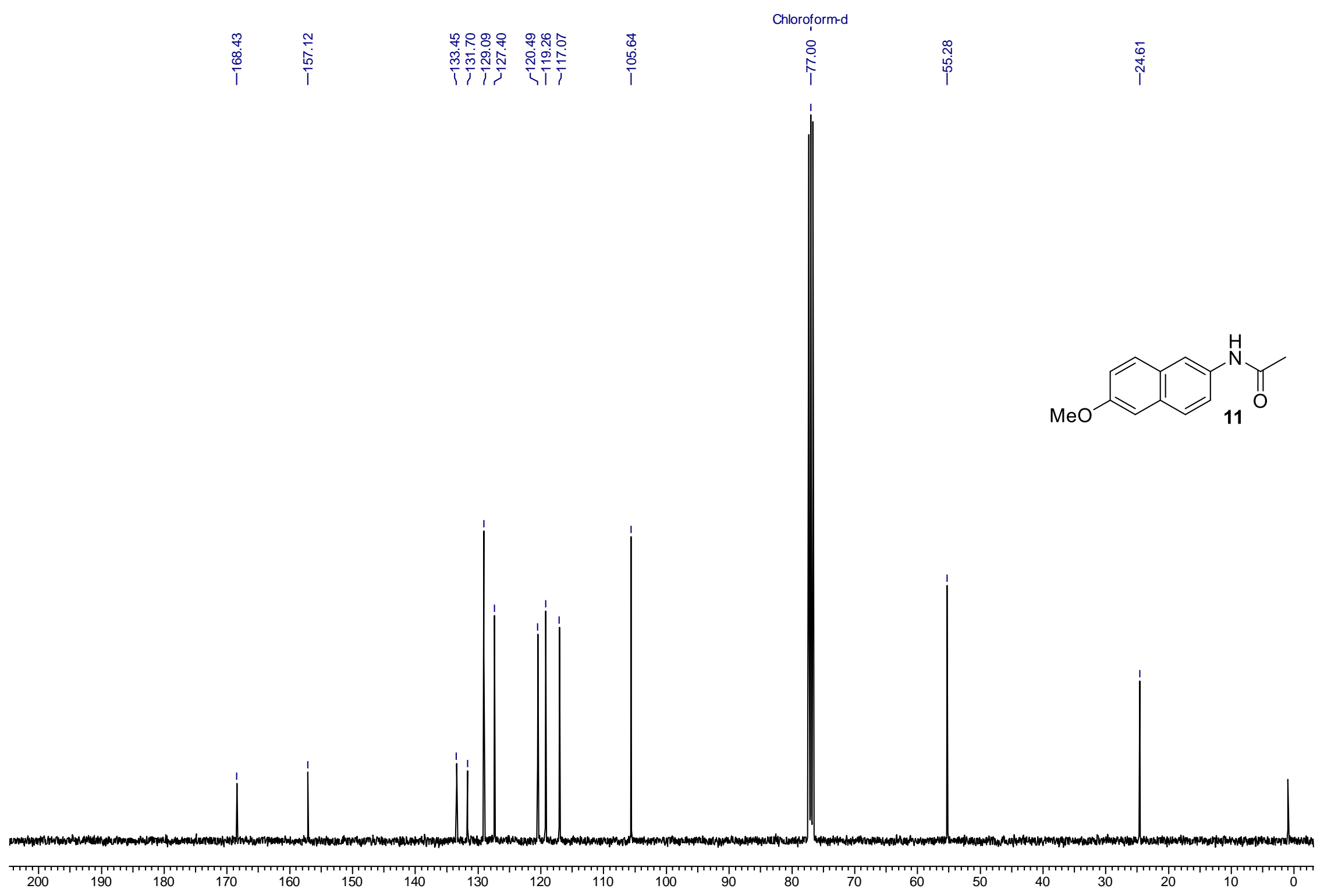




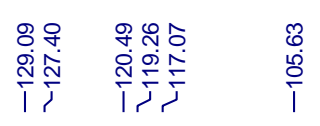

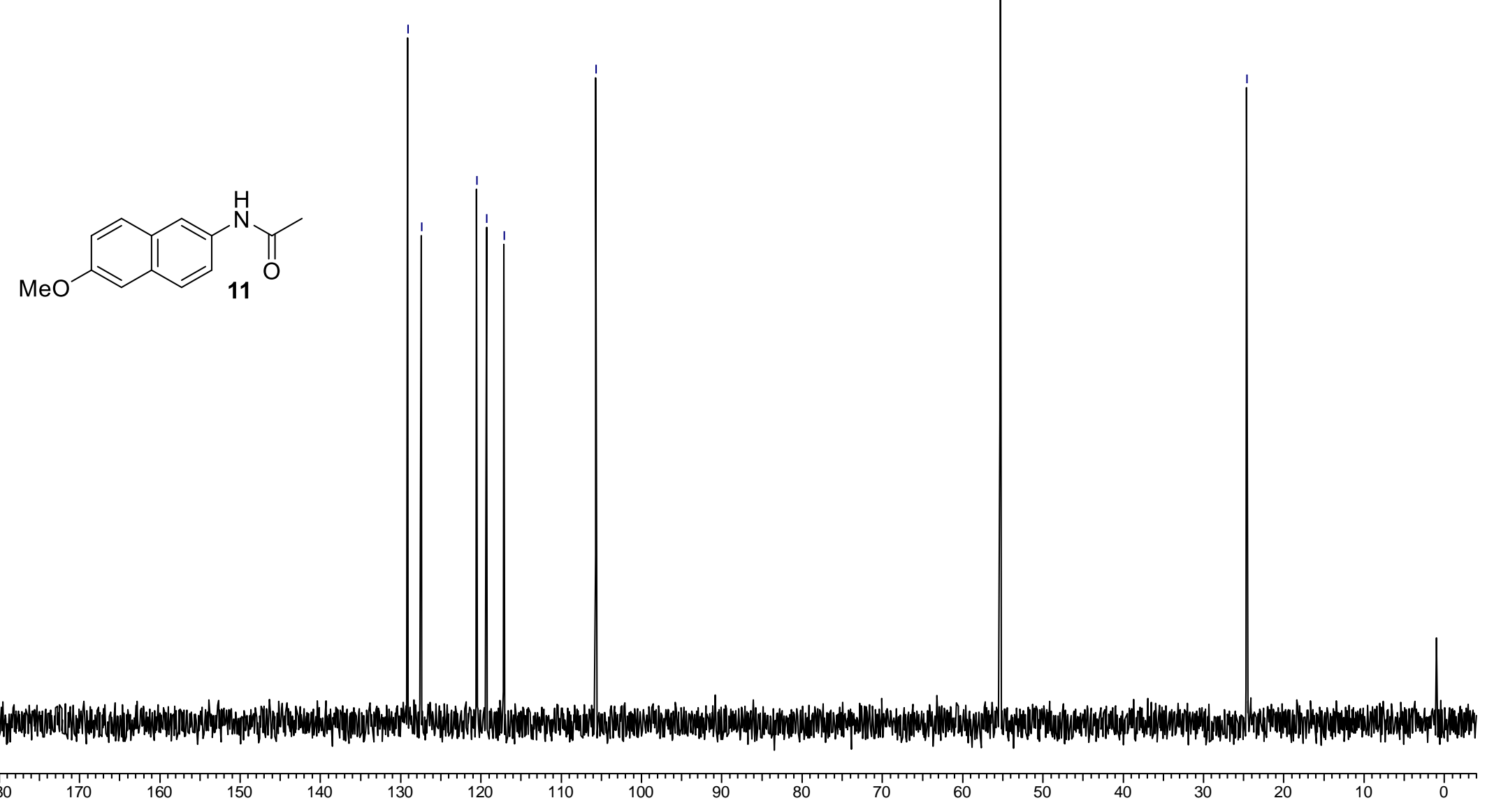

11

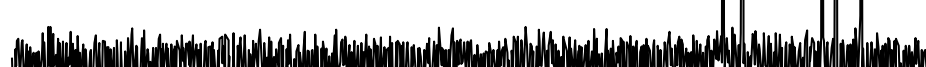




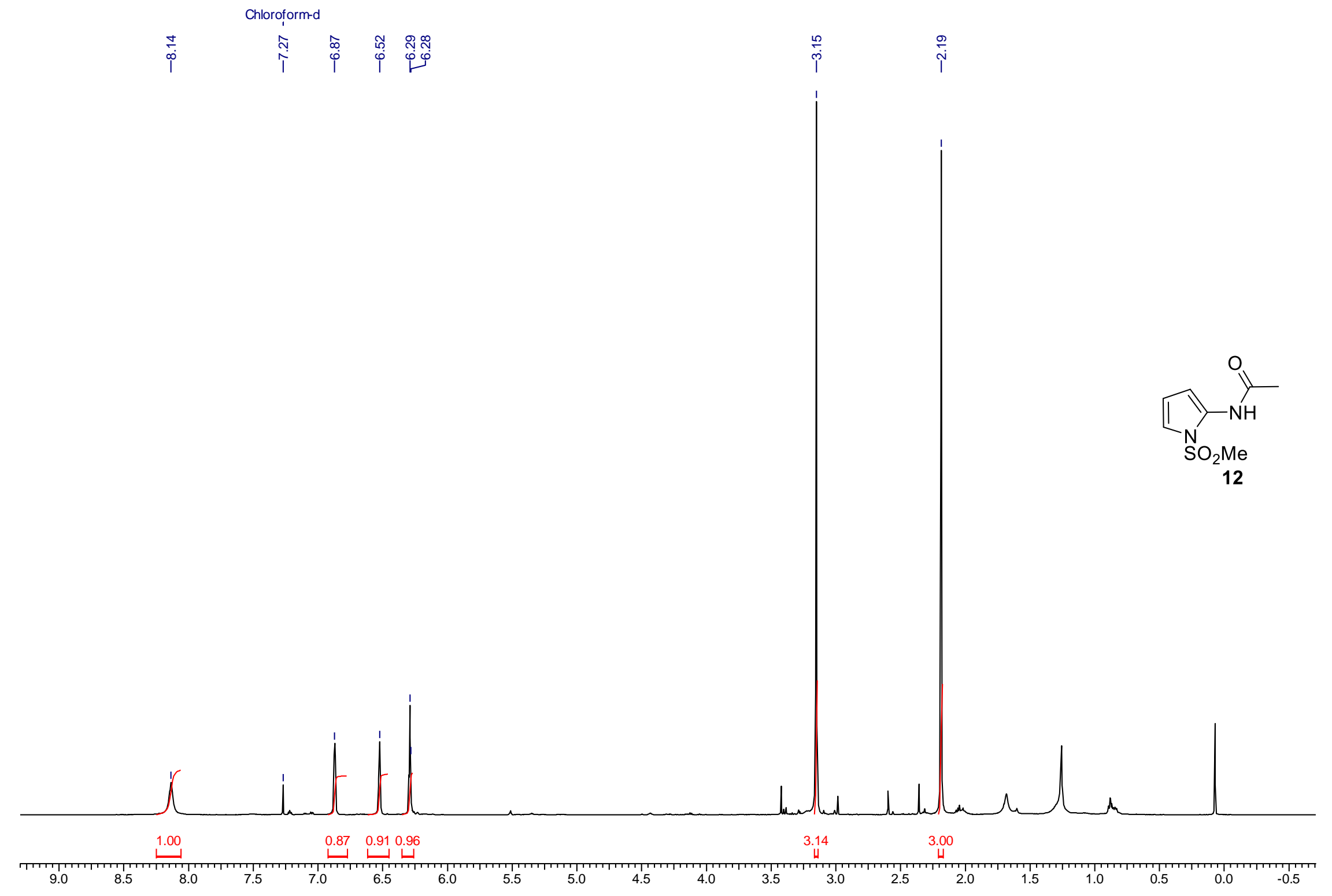


${ }^{13} \mathrm{C}$ NMR, $125 \mathrm{MHz}$

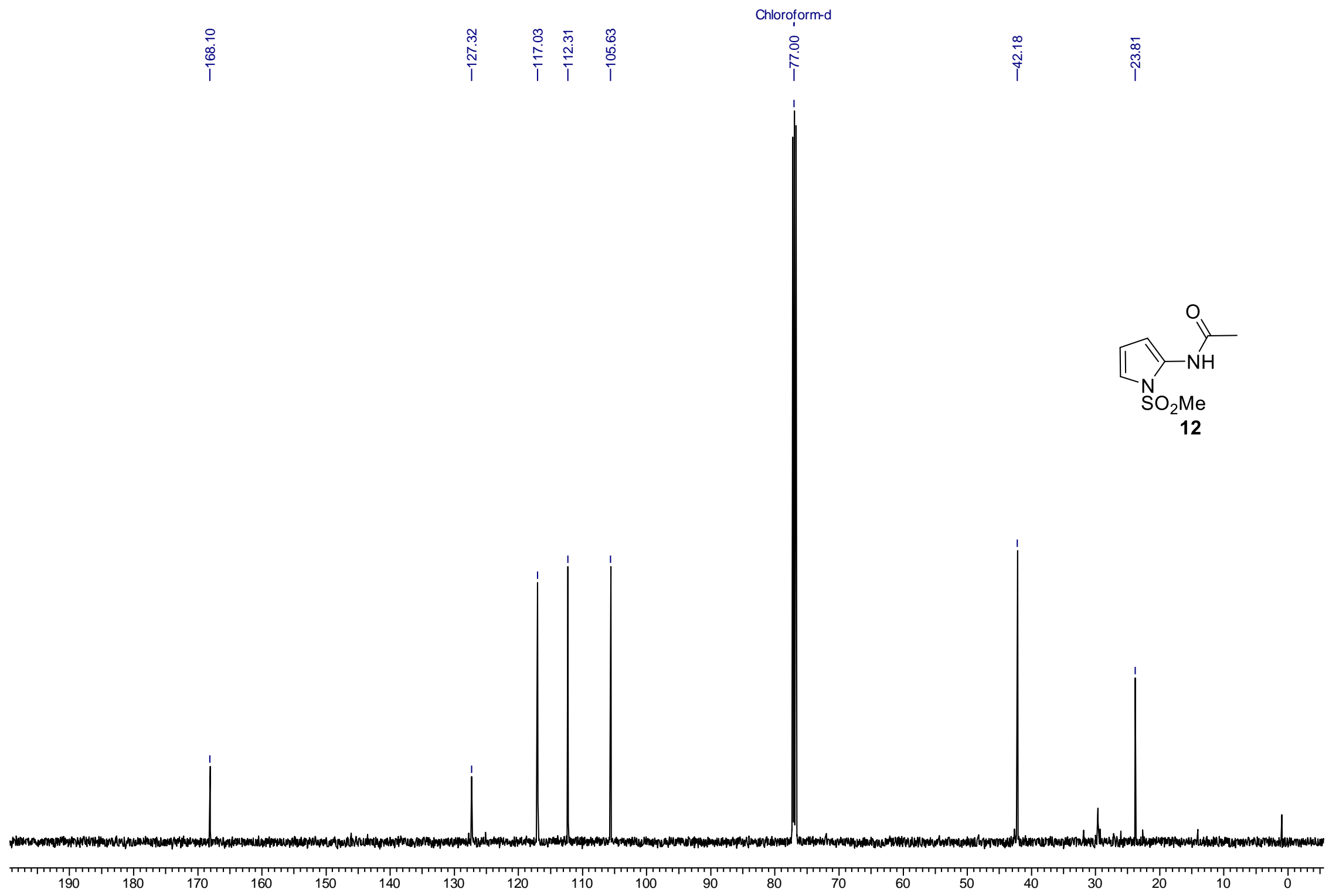

55 


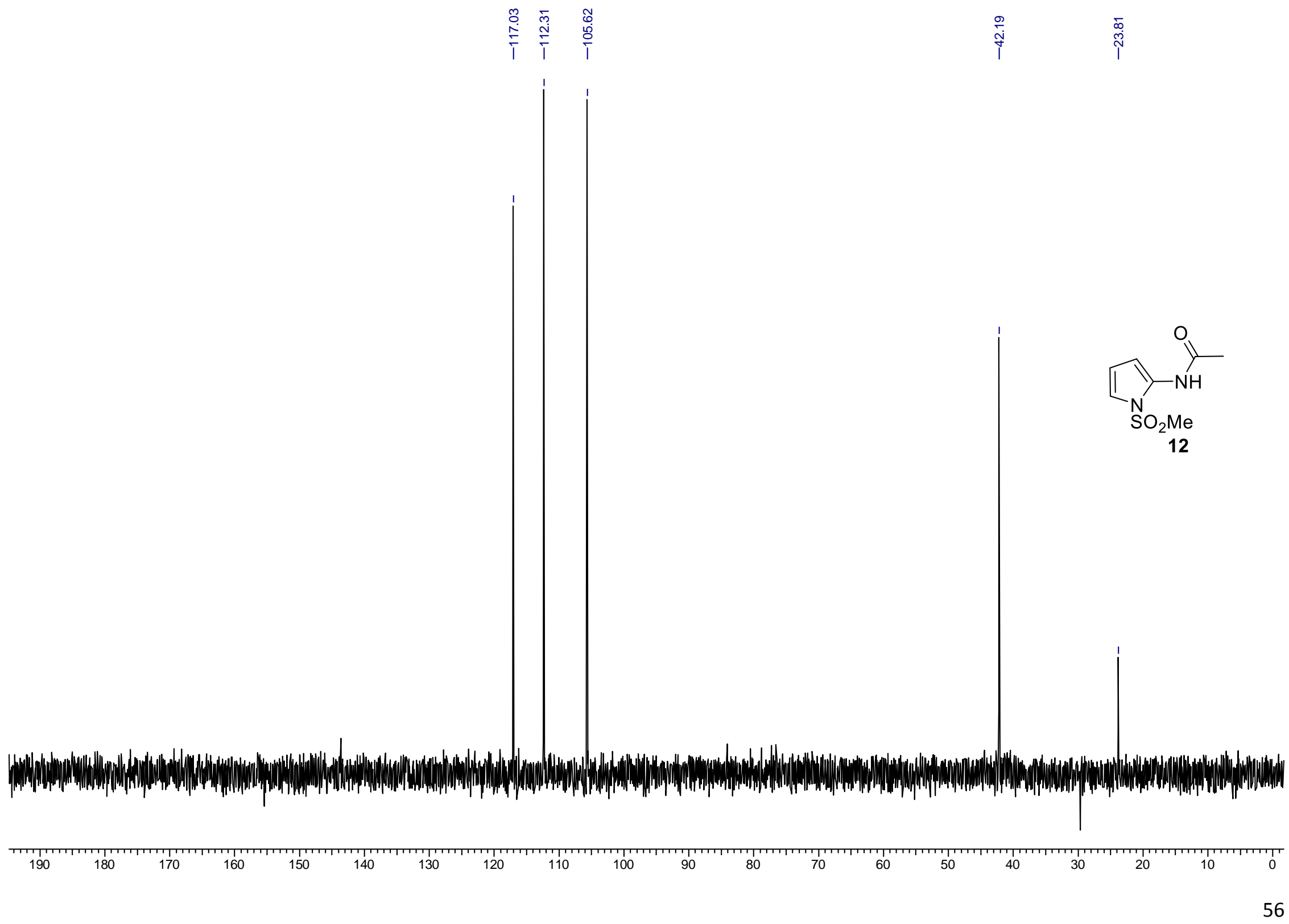




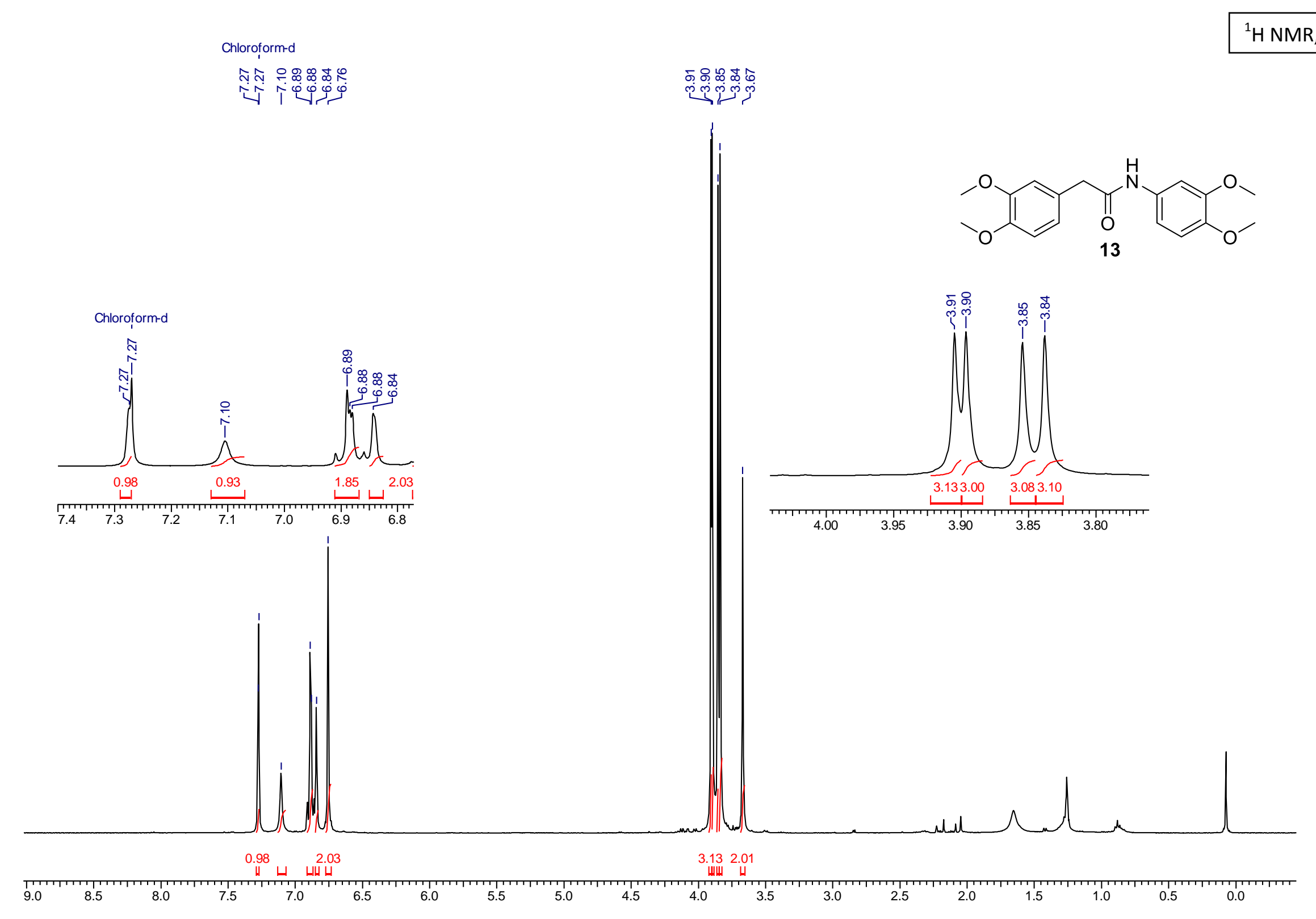




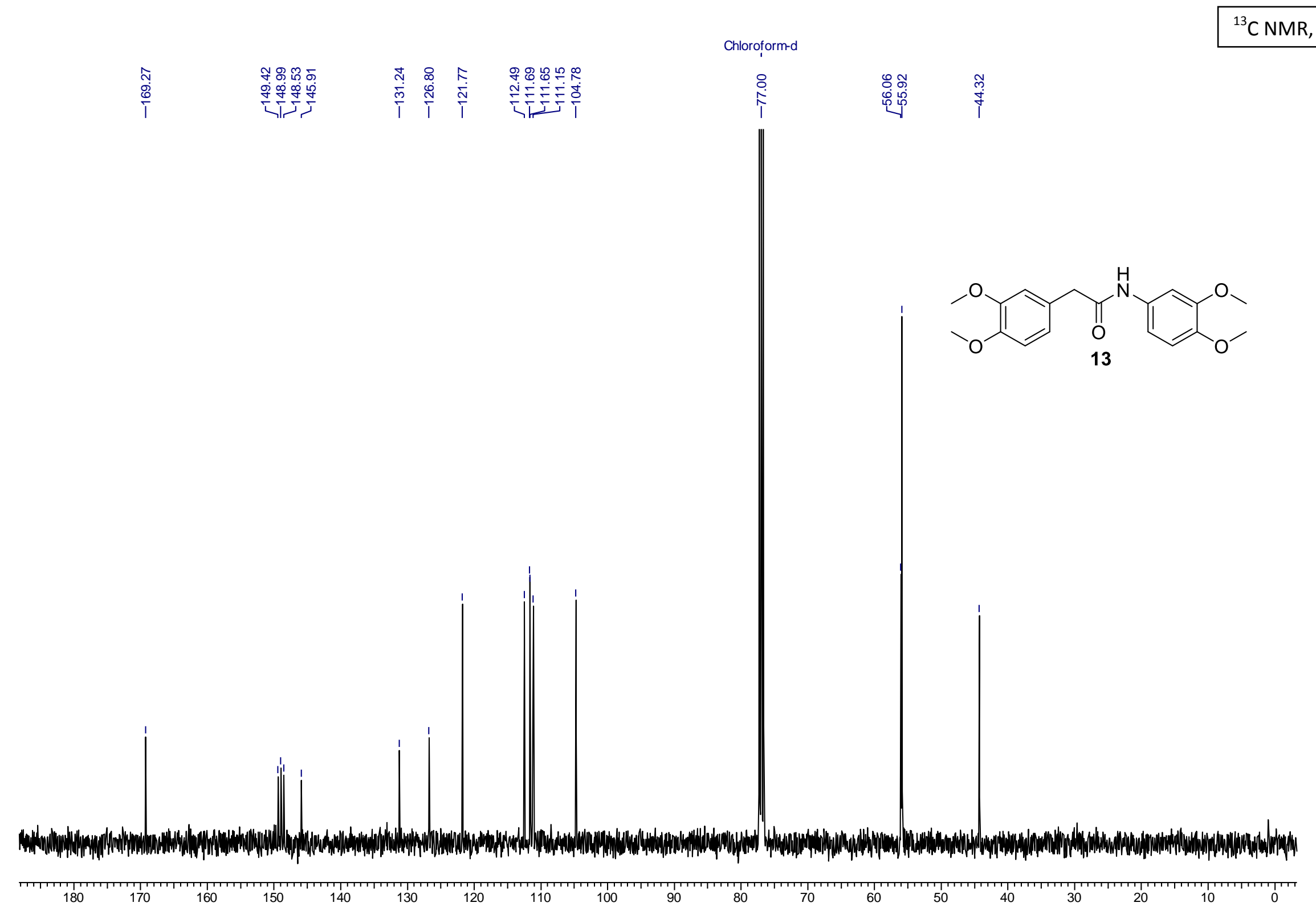




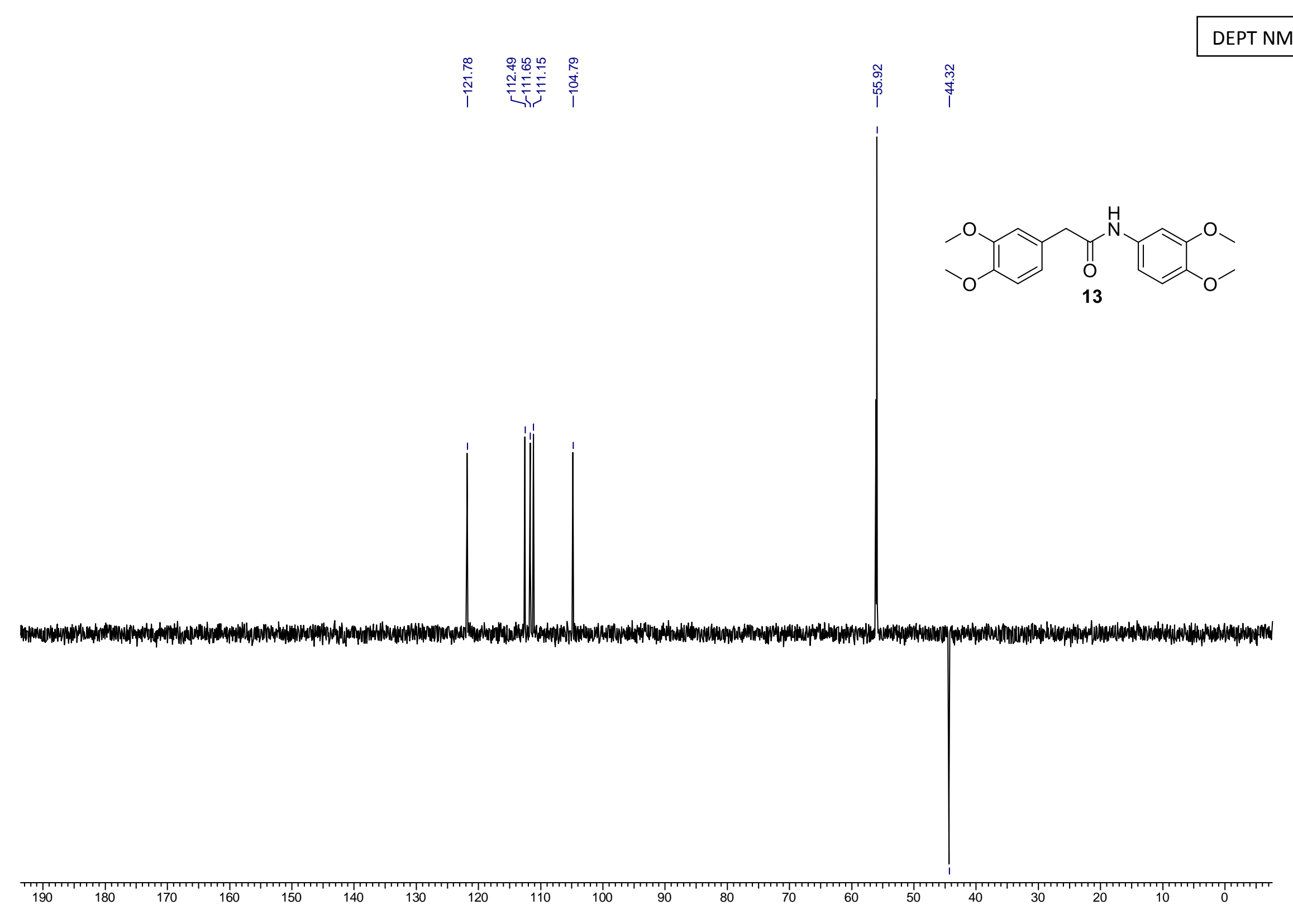


Chloroform-d

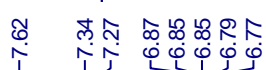

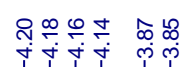

ำำ

苗
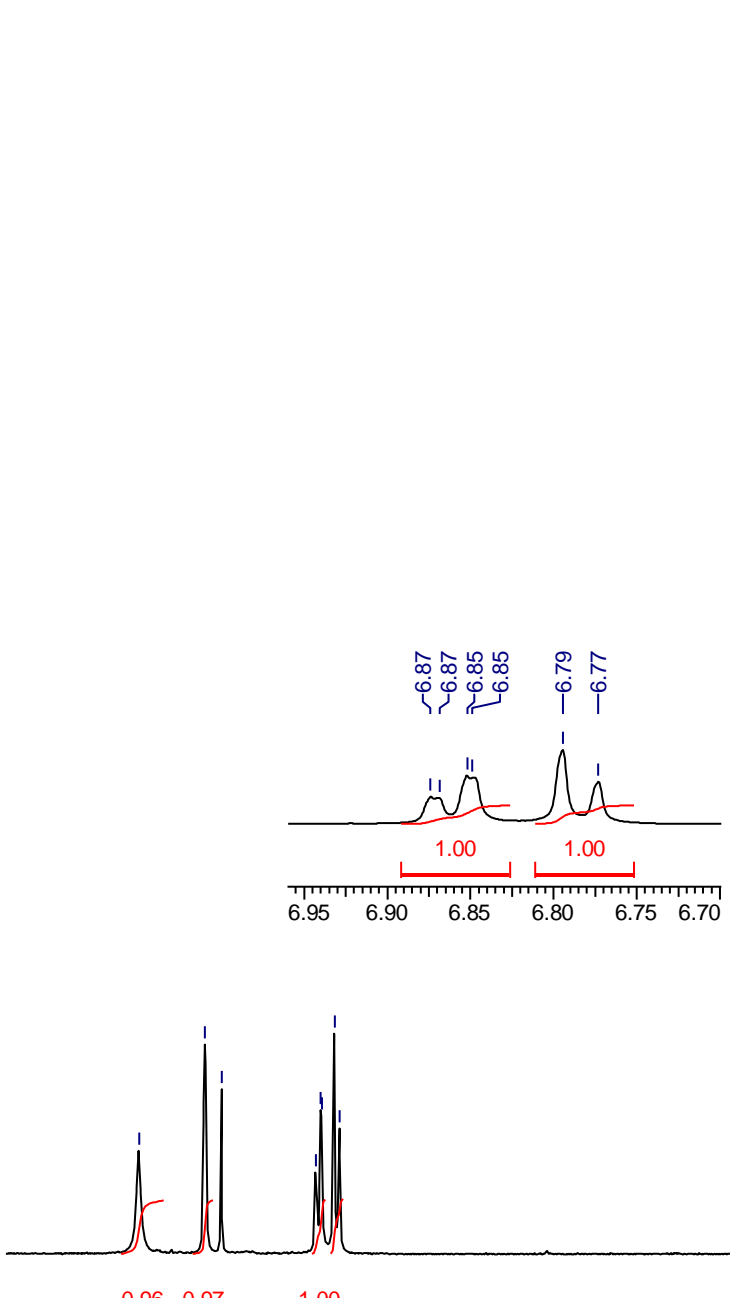

1.00 $5.5 \quad 5.0$ $5.0 \quad 4.5$
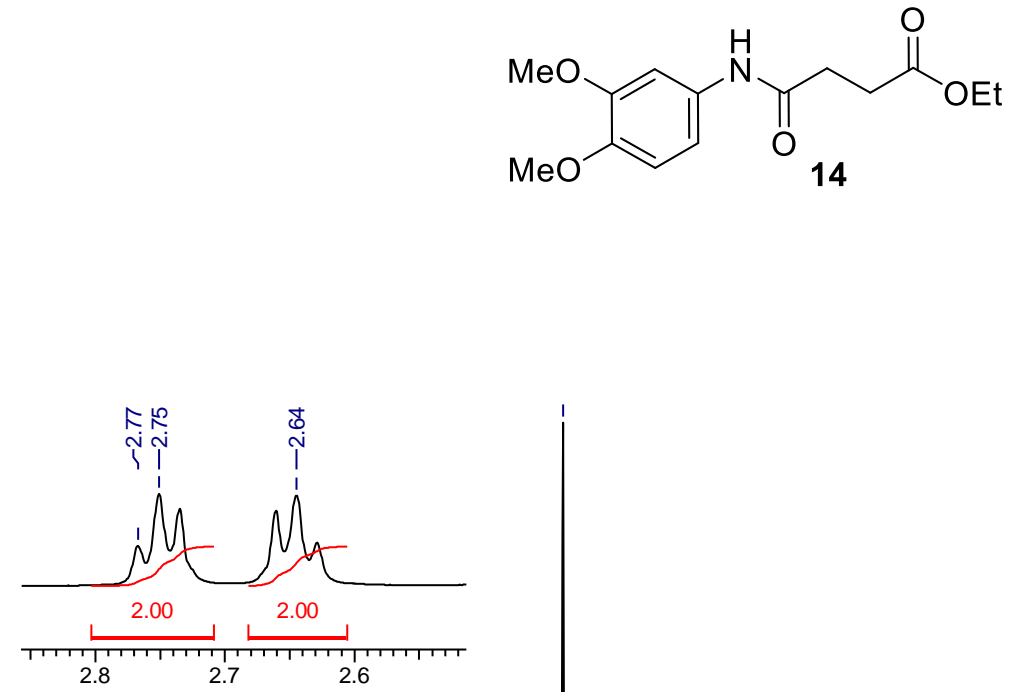
8.0 0.5
7.5
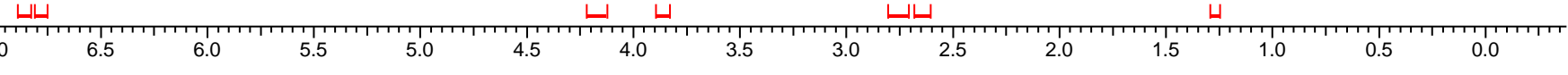


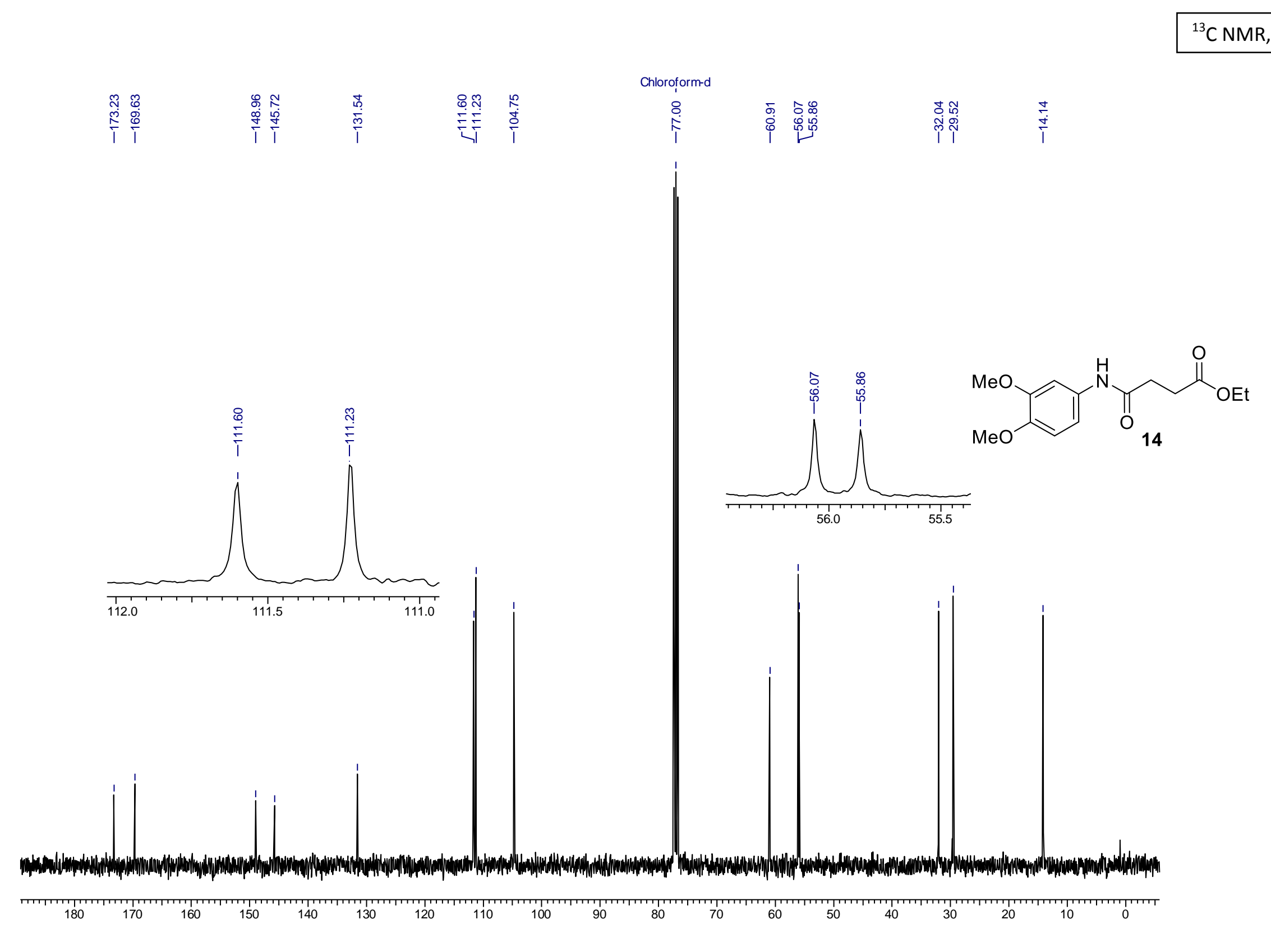




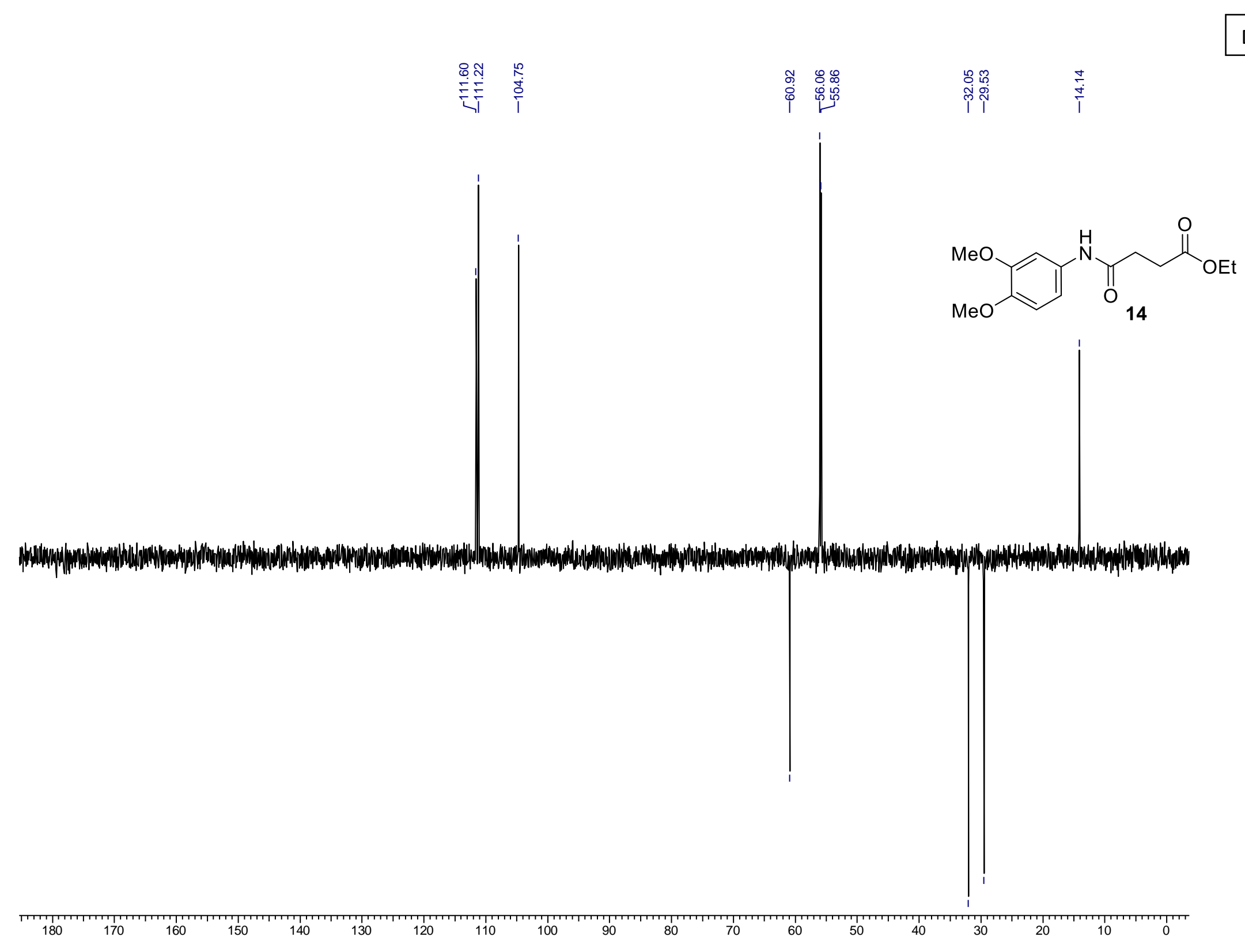


Chloroform-d

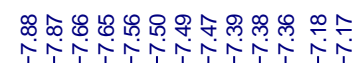
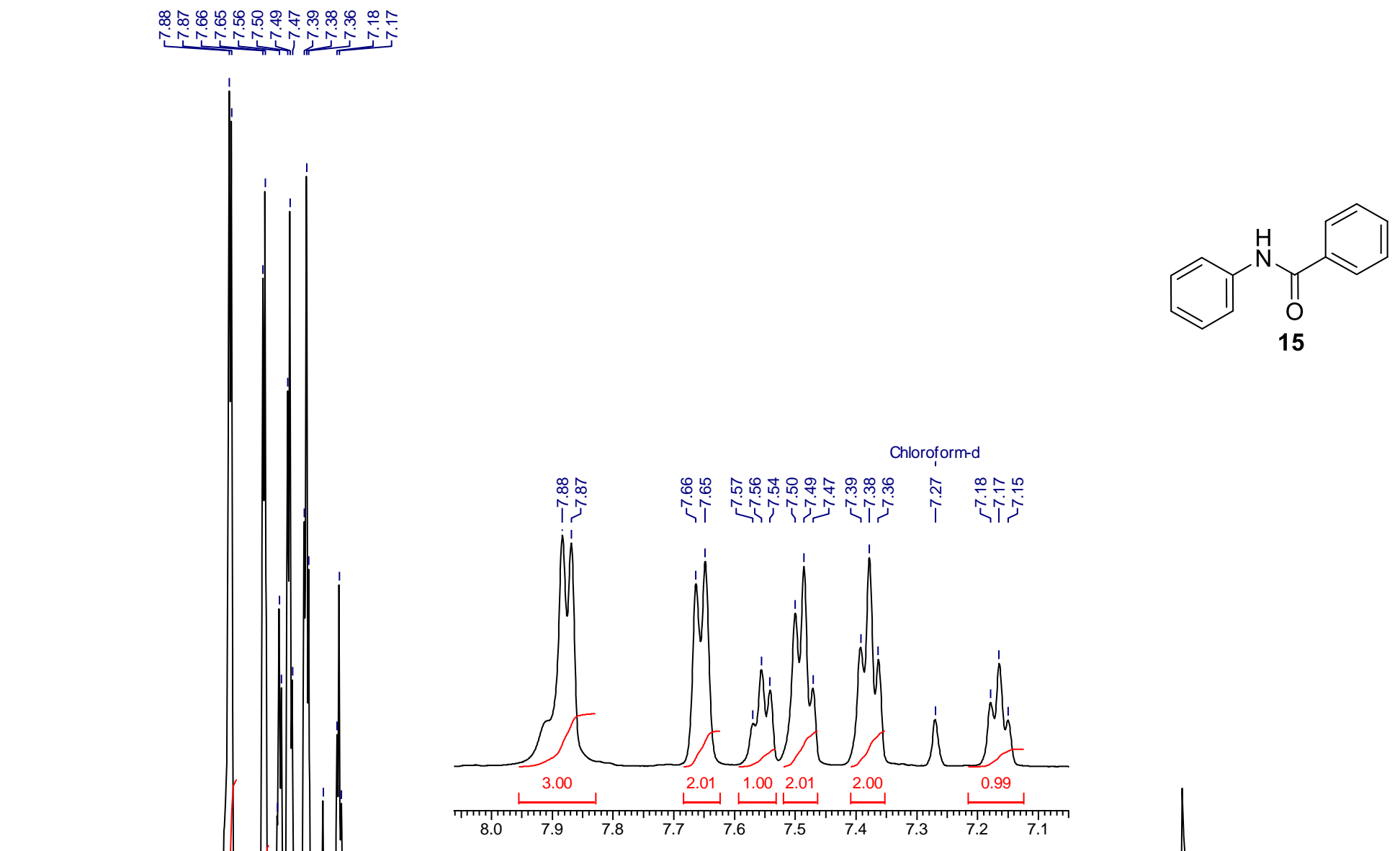


\section{б. 8 œ ๓}

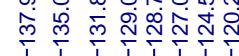

Chloroform-d

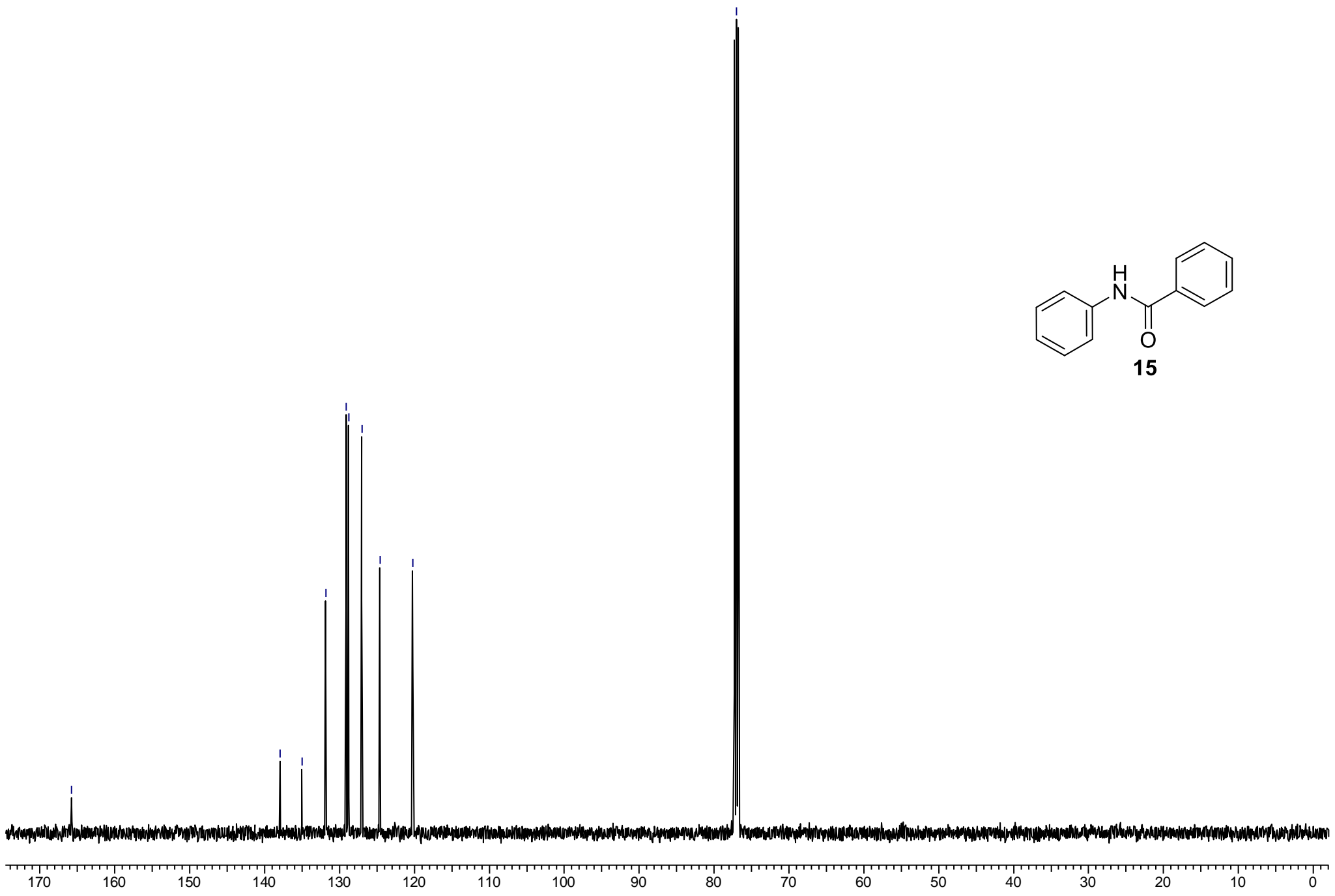




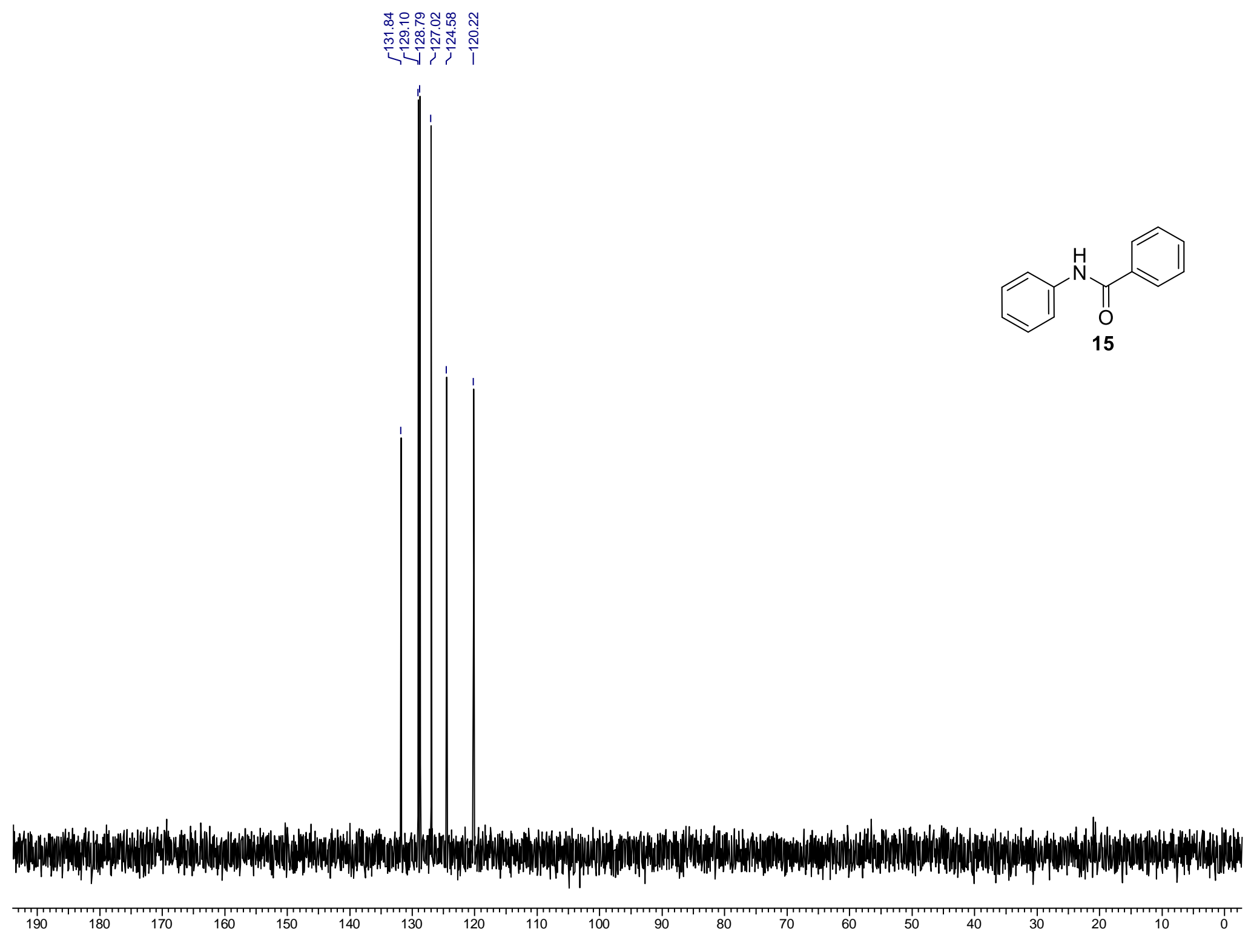

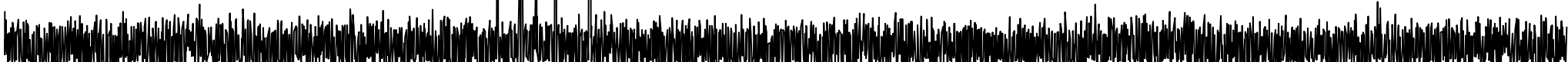




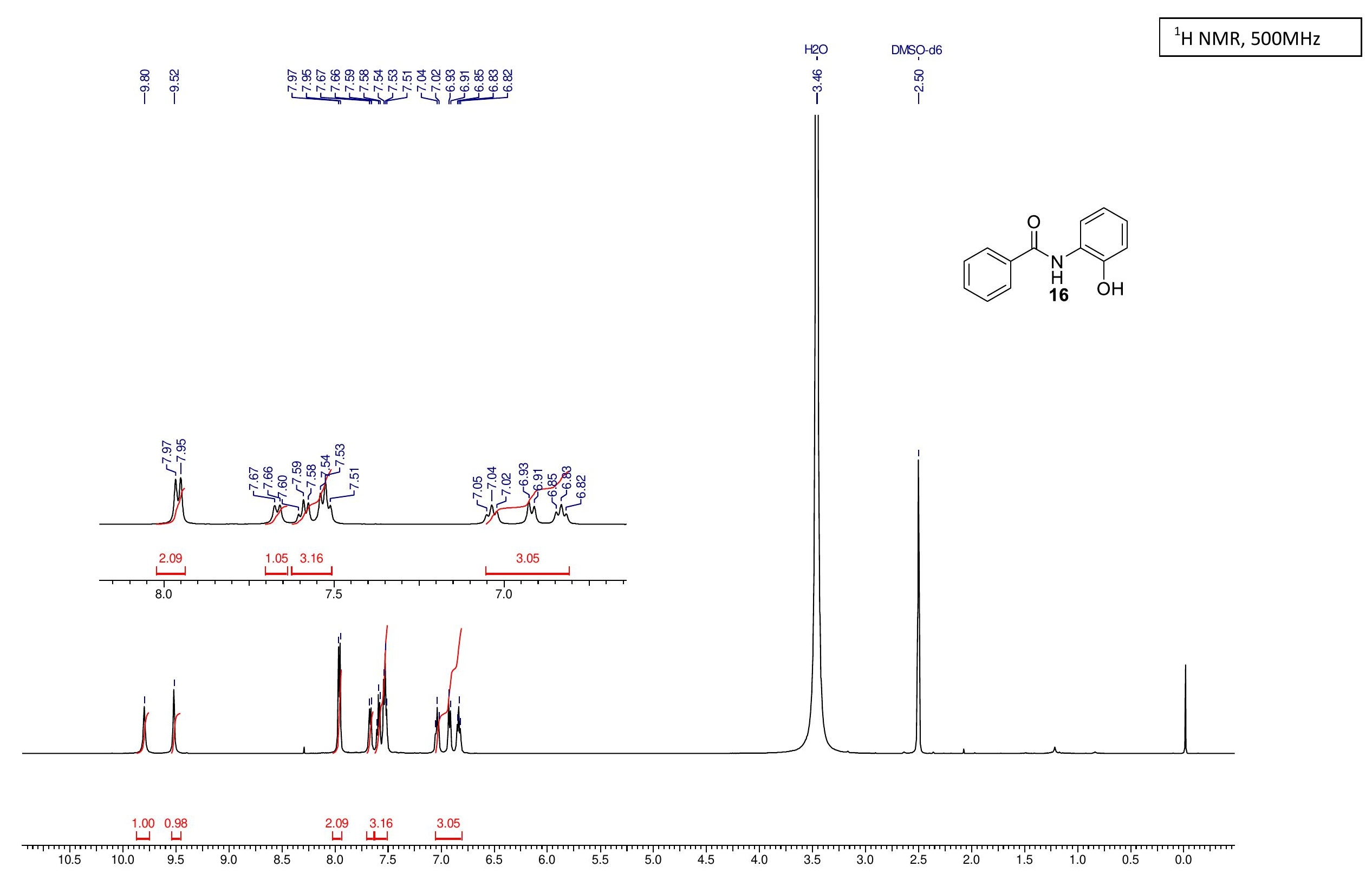



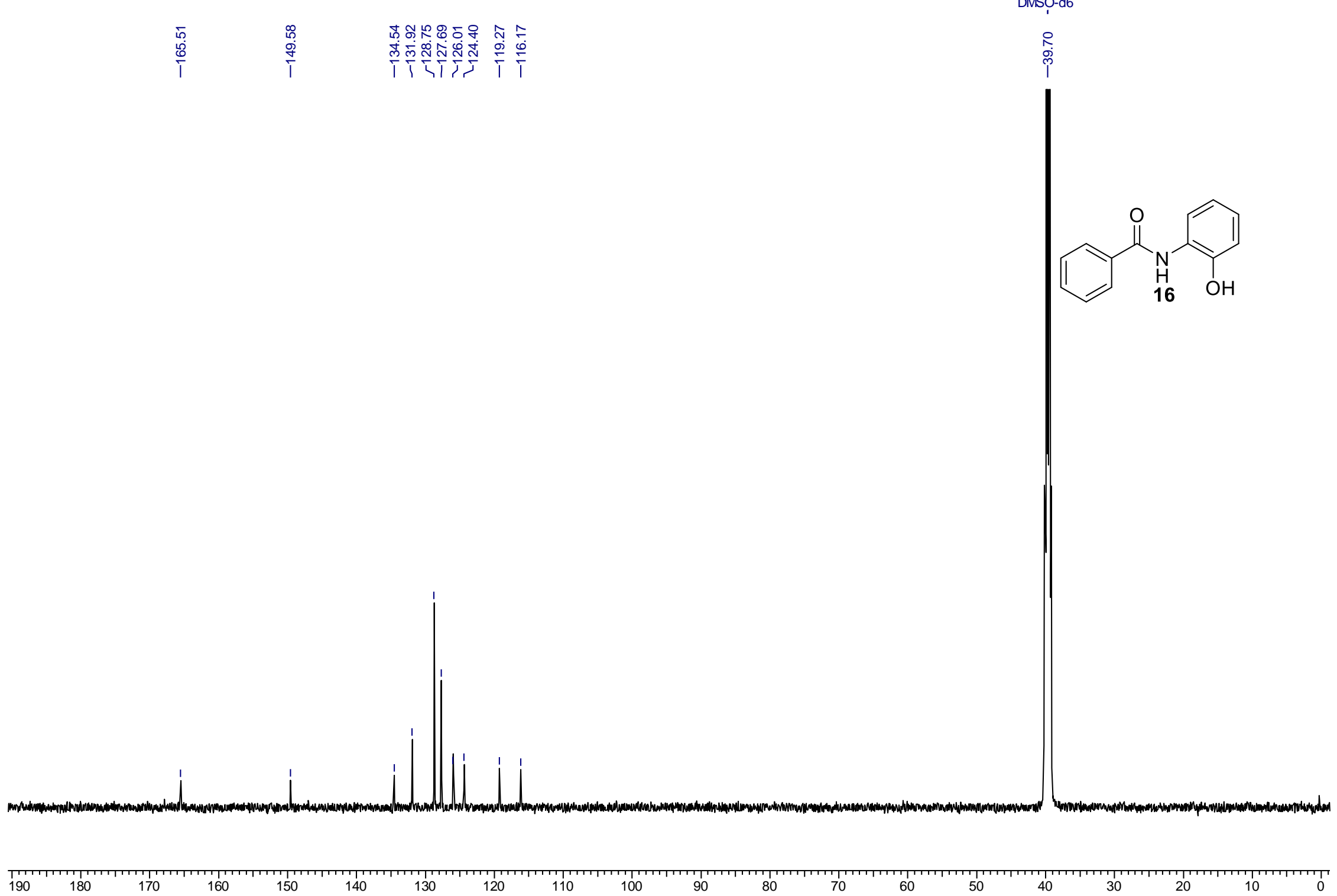


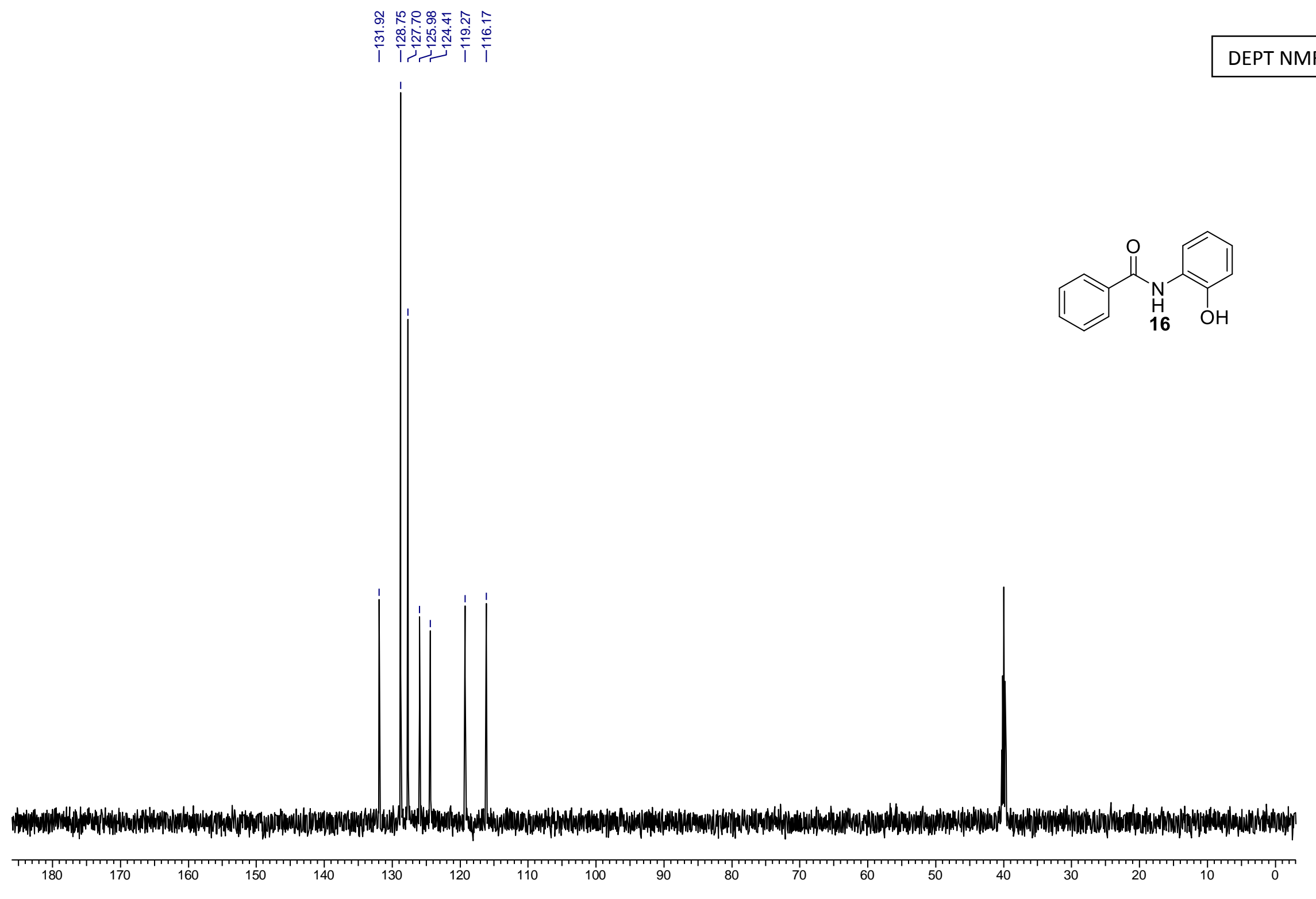




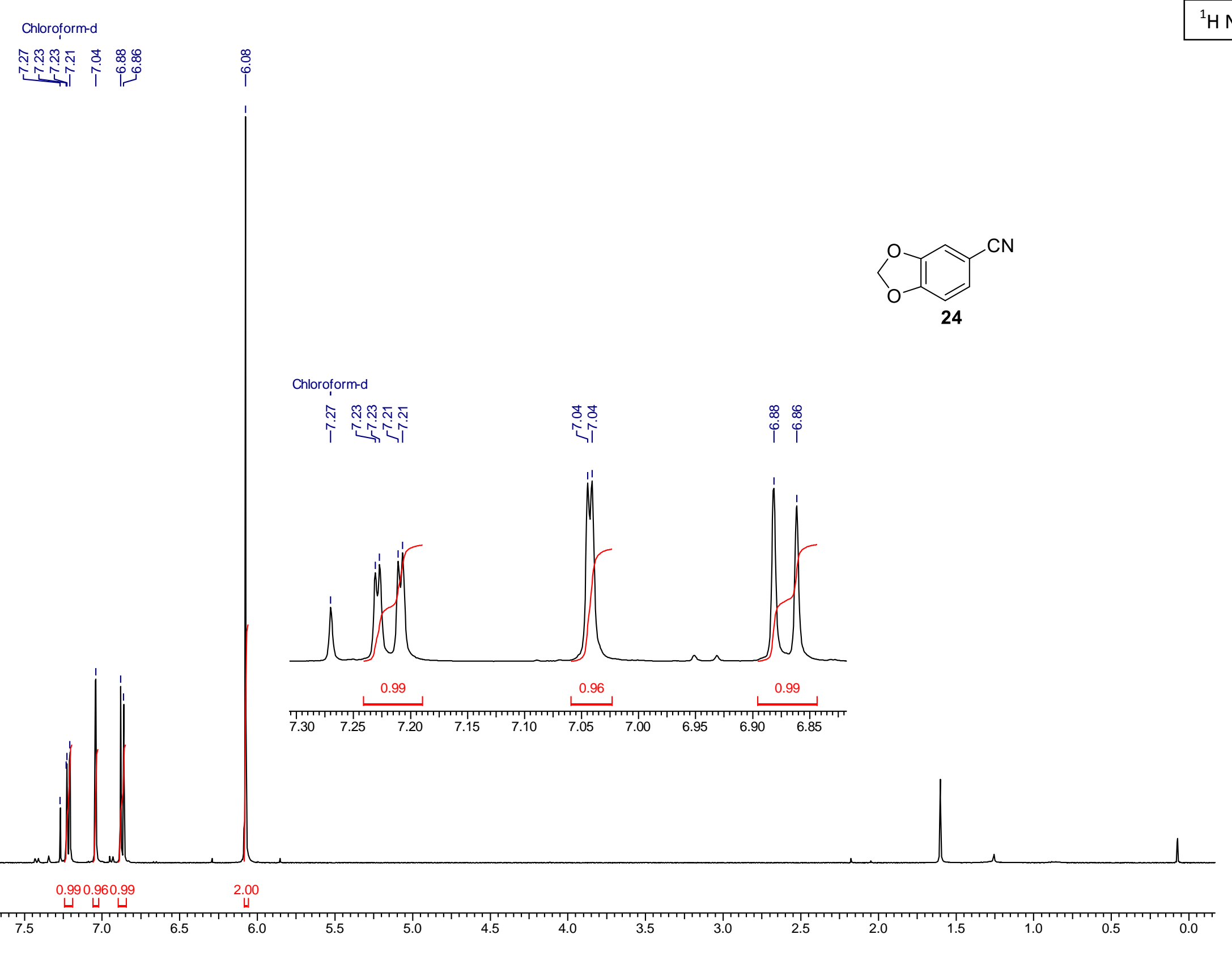


${ }^{13} \mathrm{C}$ NMR, 100MHz

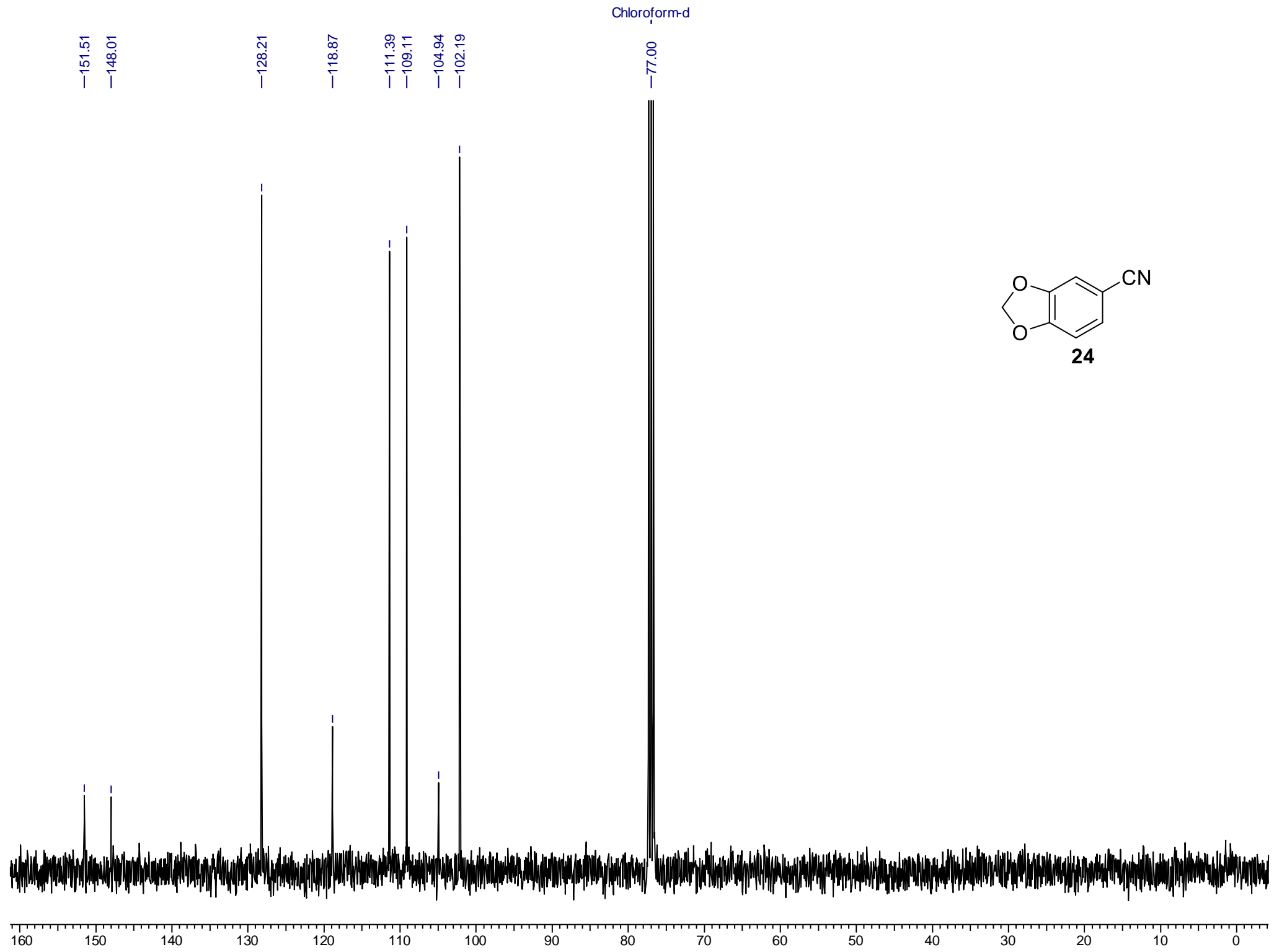

70 


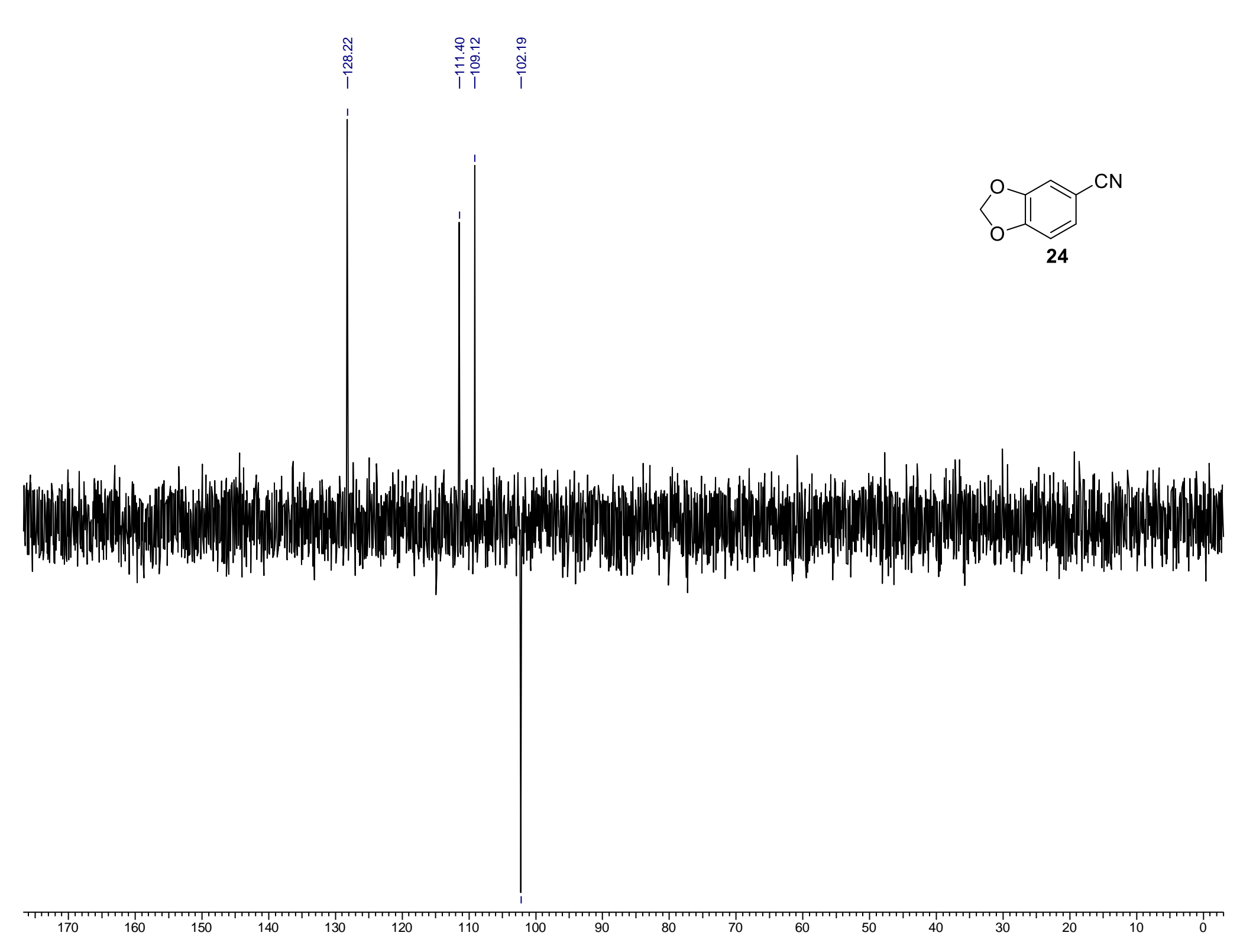


${ }^{1} \mathrm{H}$ NMR, 400MHz

Chloroform-d

ヘิ

$\underset{\infty}{\infty}$
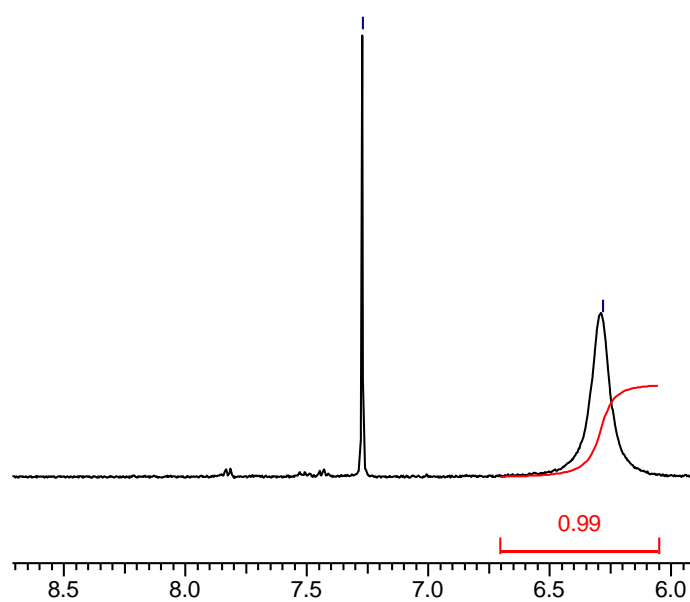

7.0

$5.5 \quad 5.0$

$4.5 \quad 4.0$

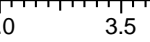

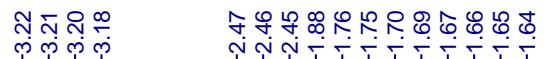

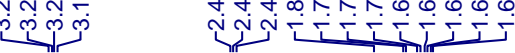

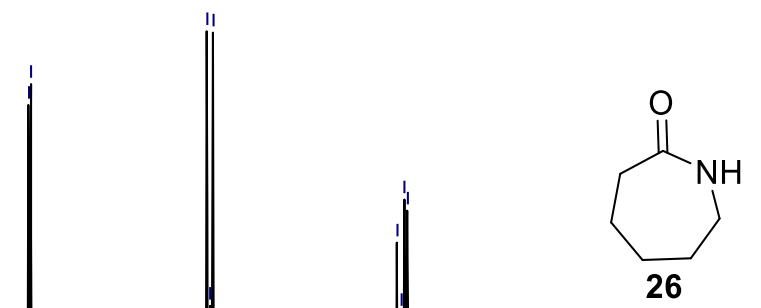


Chloroform-d

$$
\begin{aligned}
& \stackrel{8}{i}
\end{aligned}
$$

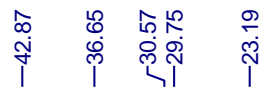

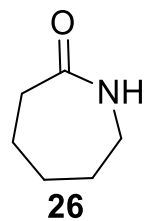
${ }_{190}$ 


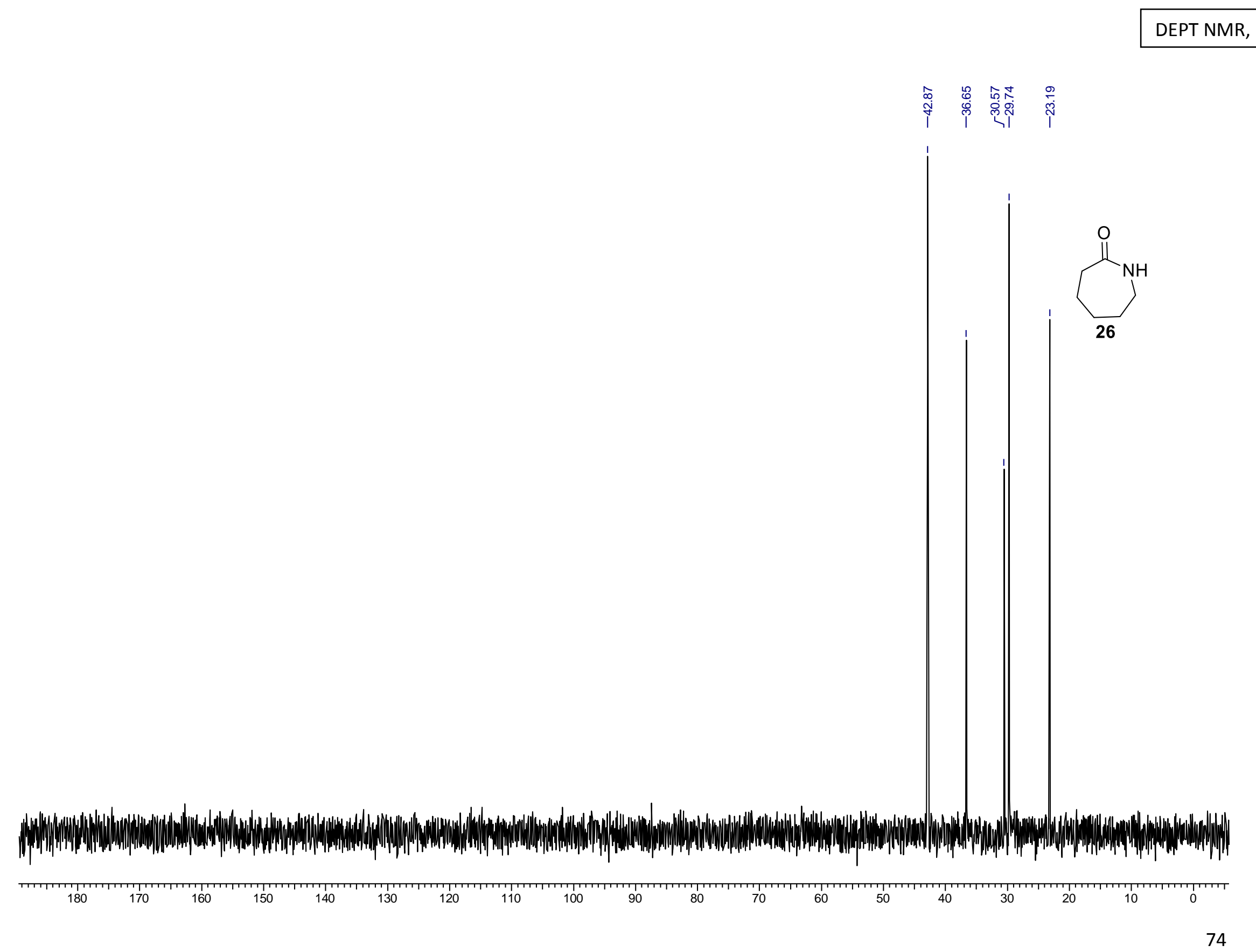




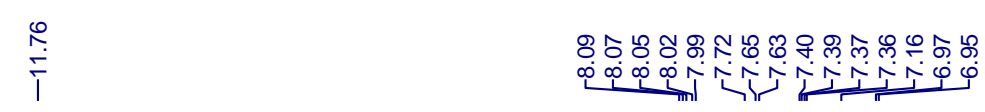

DMSO-d6

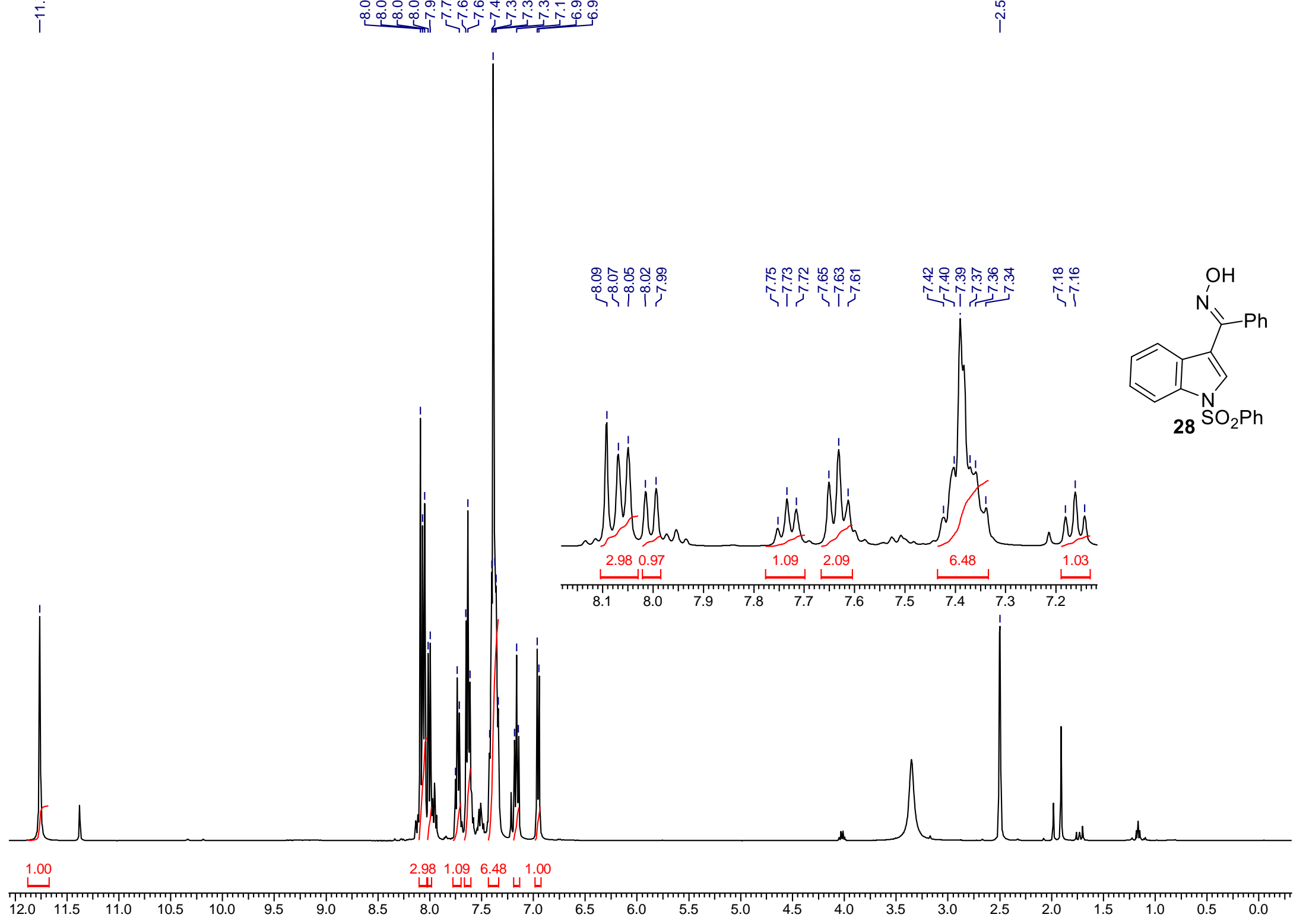



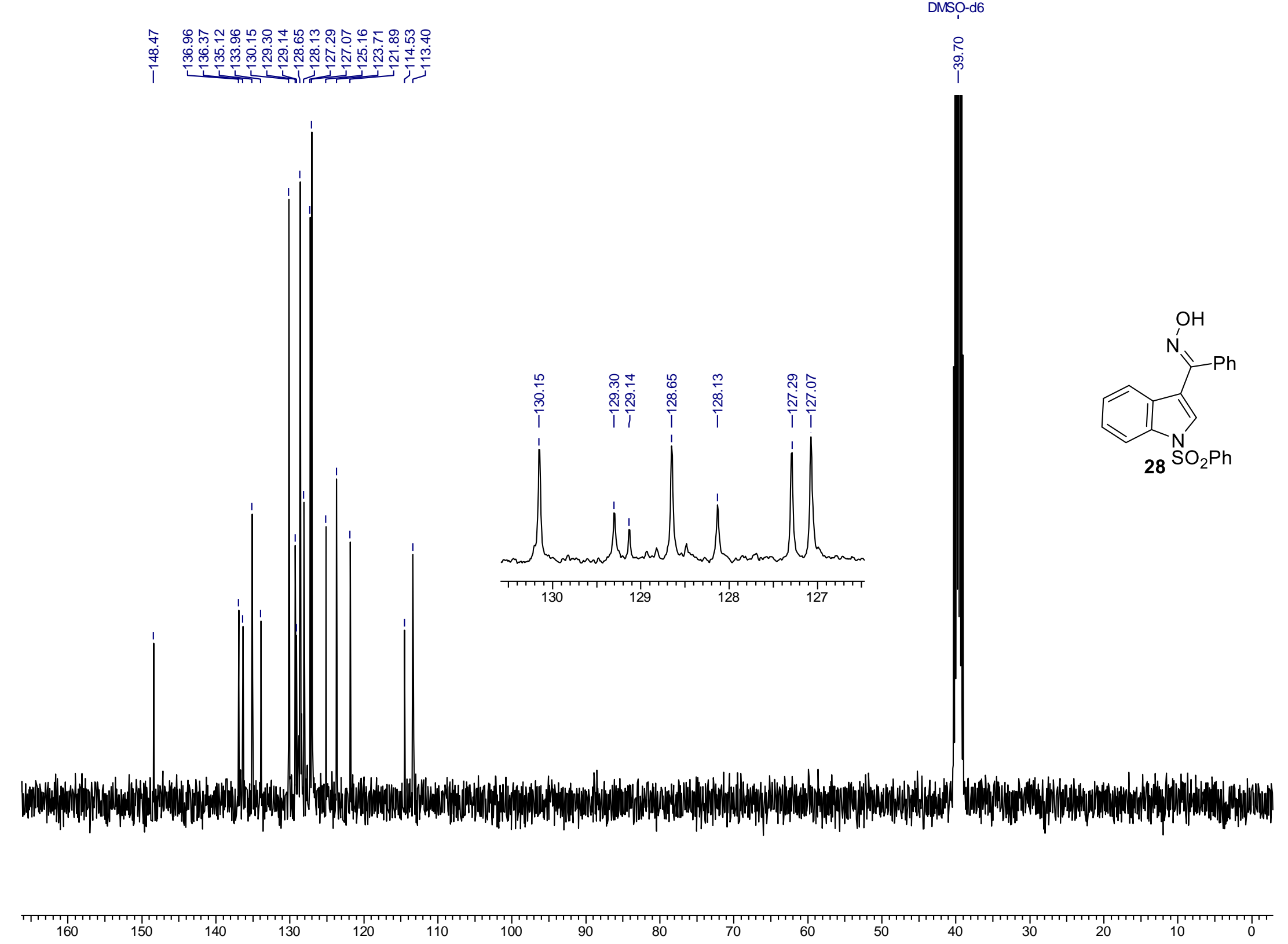


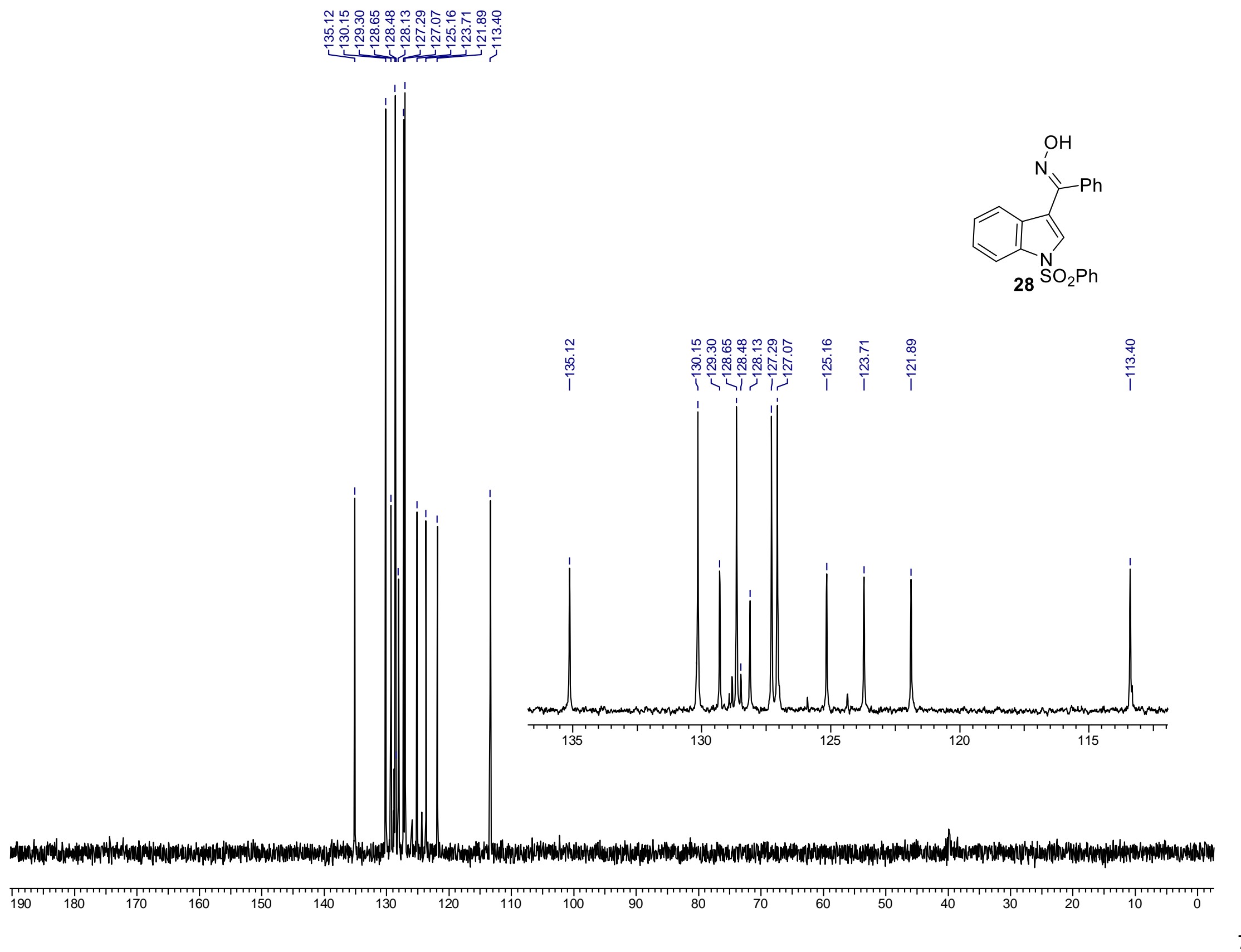


Chloroform-d

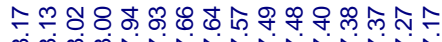

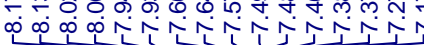

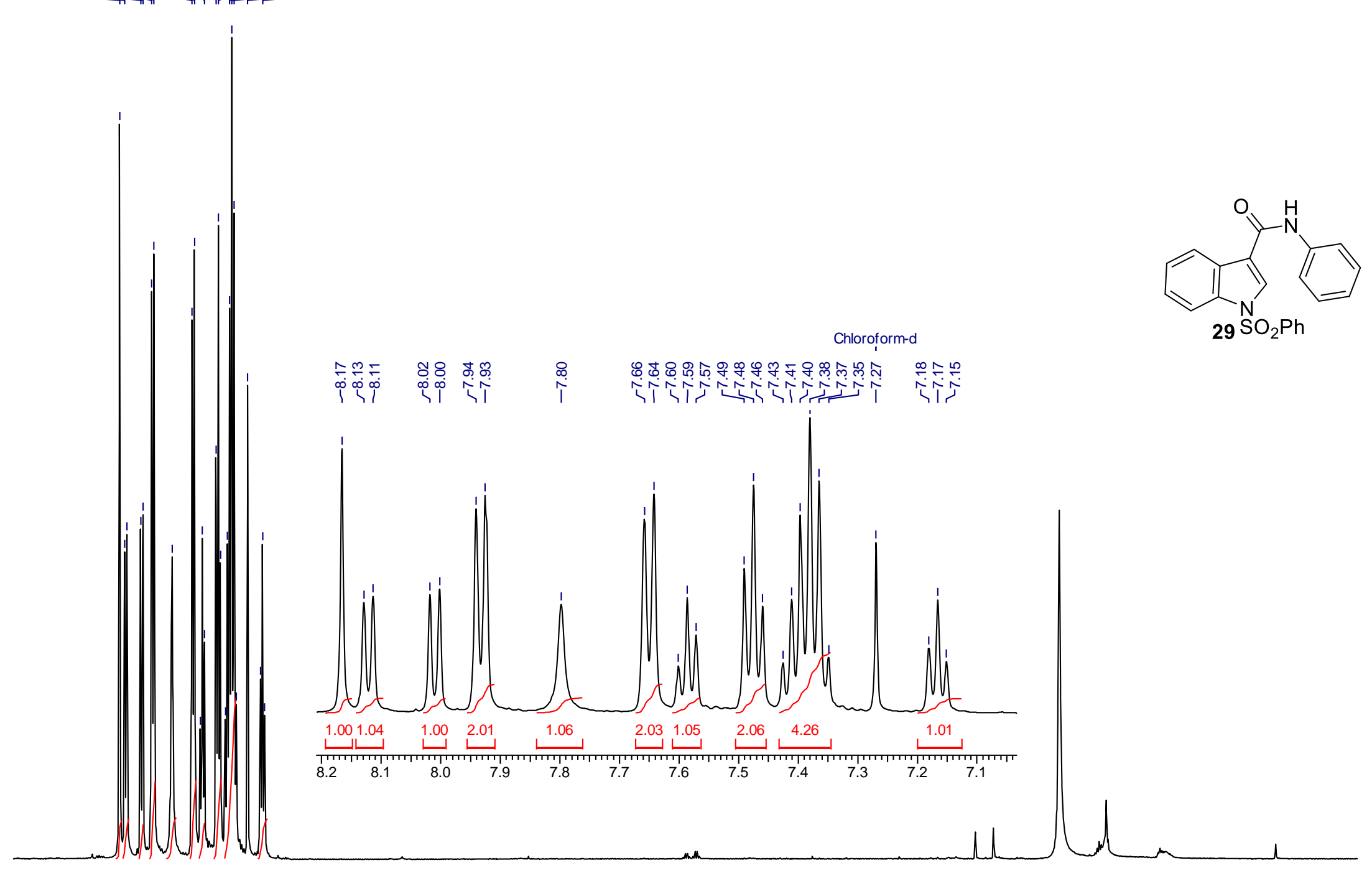

$\begin{array}{llll}1.002 .01 \quad 2.03 \quad 4.26 & 1.01\end{array}$

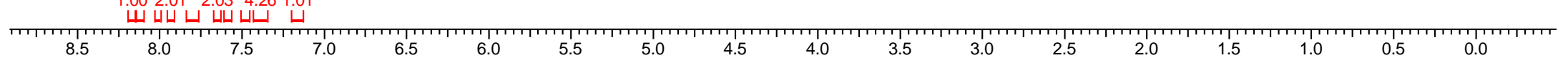




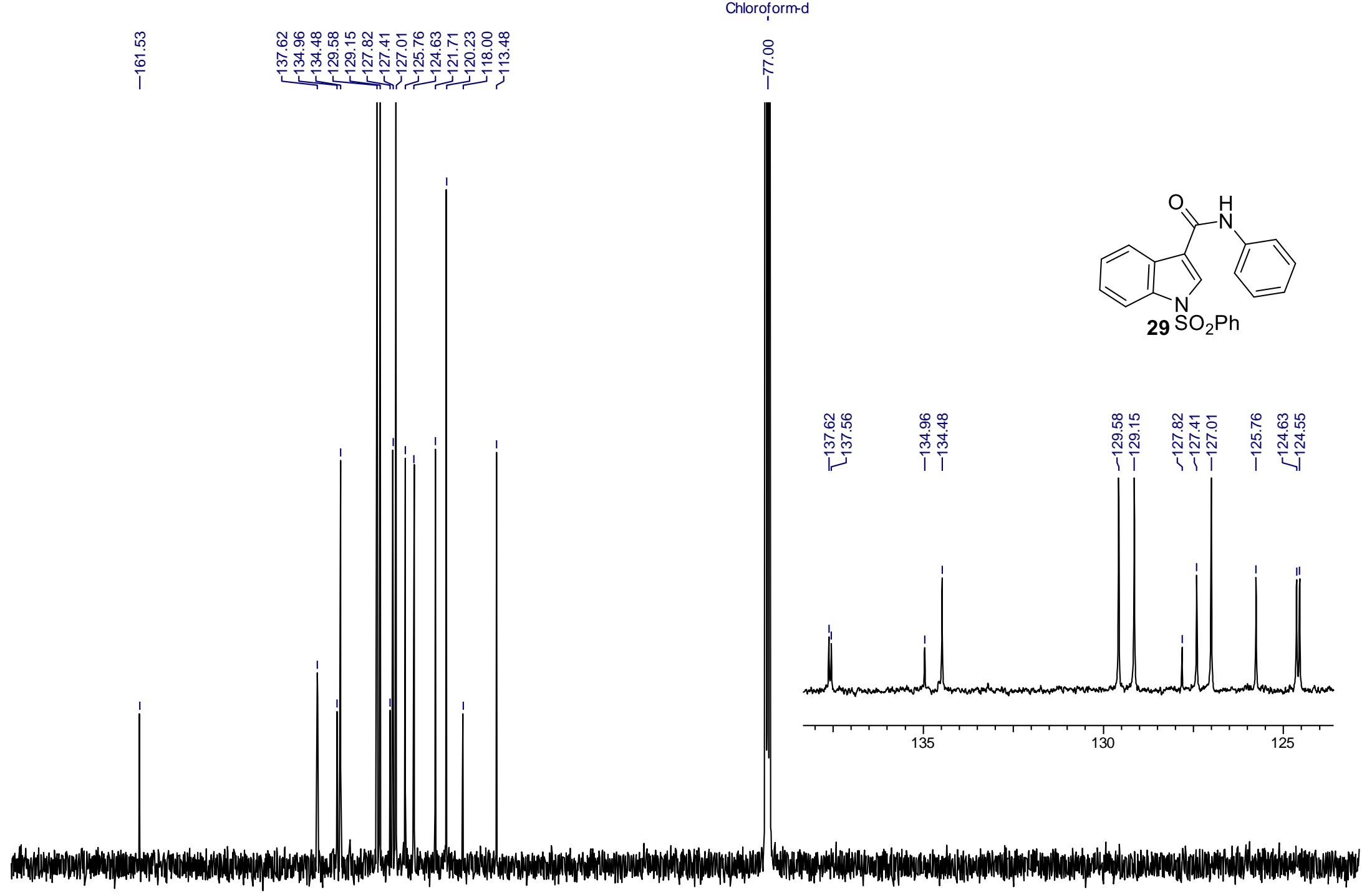




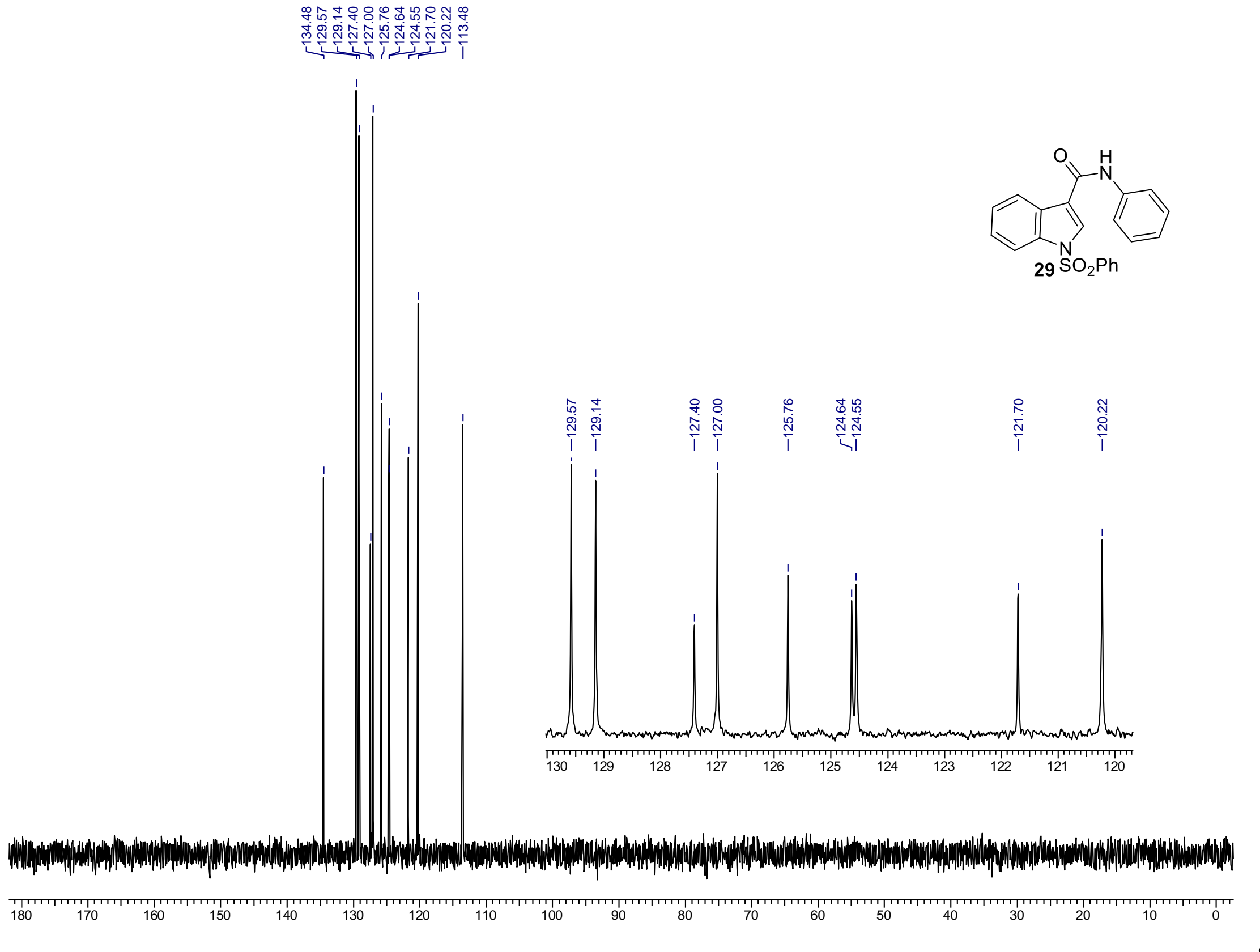




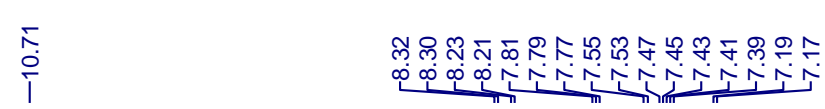

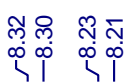
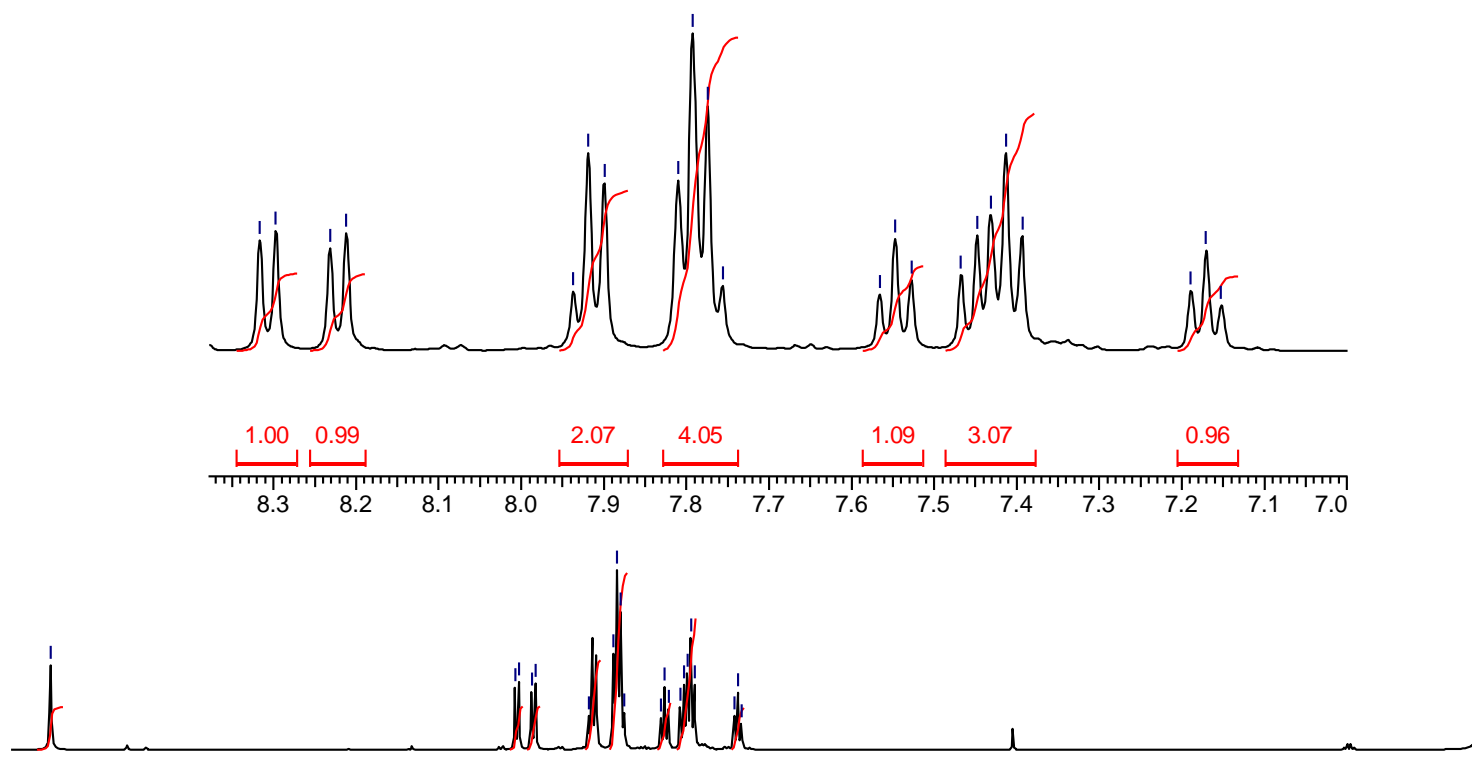

0.98

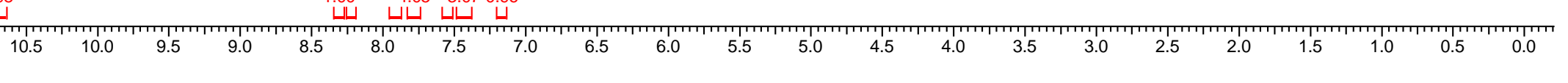

DMSO-d6

กำ

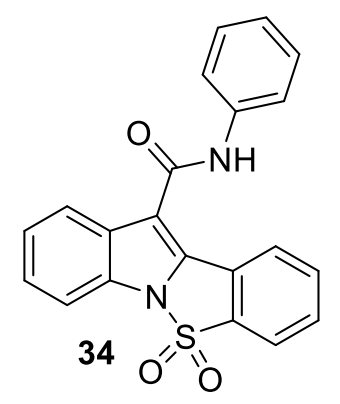




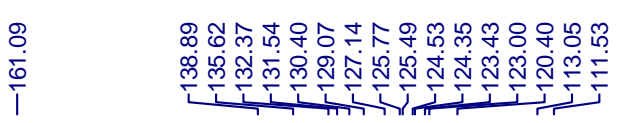

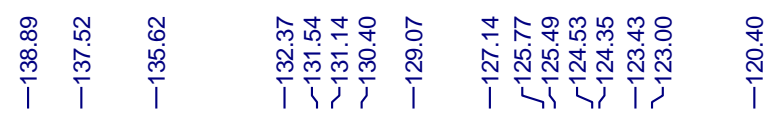
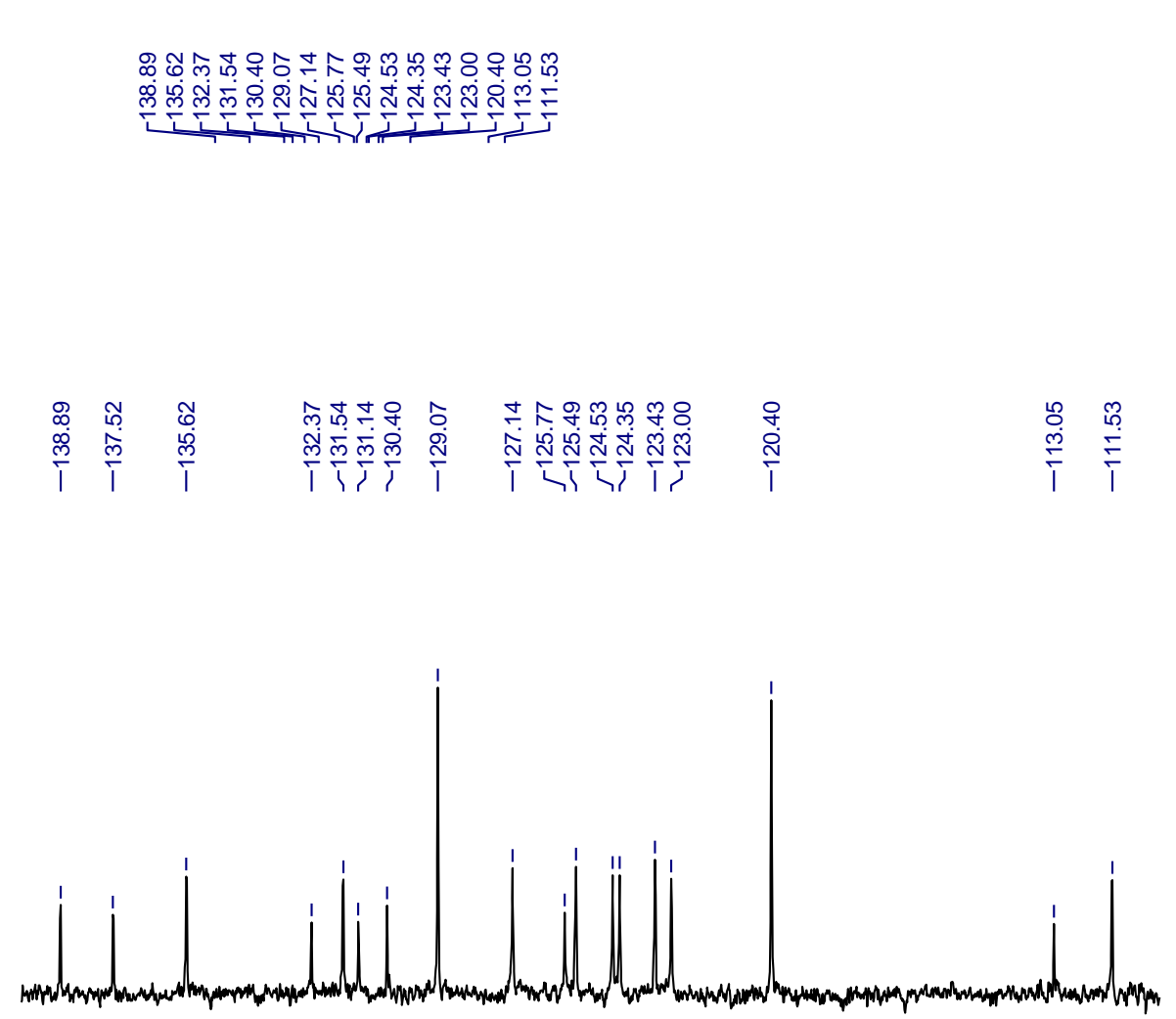

DMSO-d6

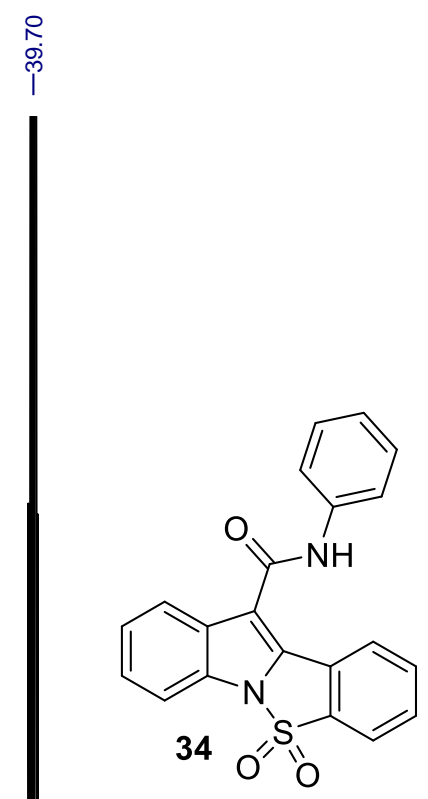

O
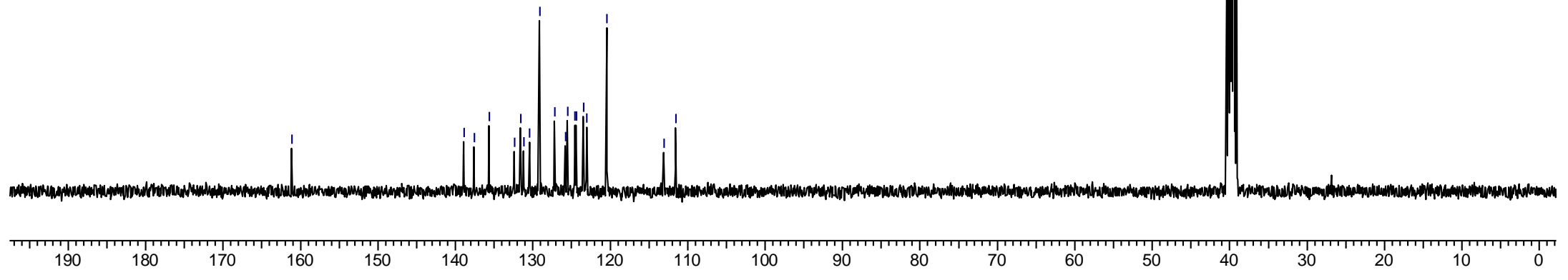


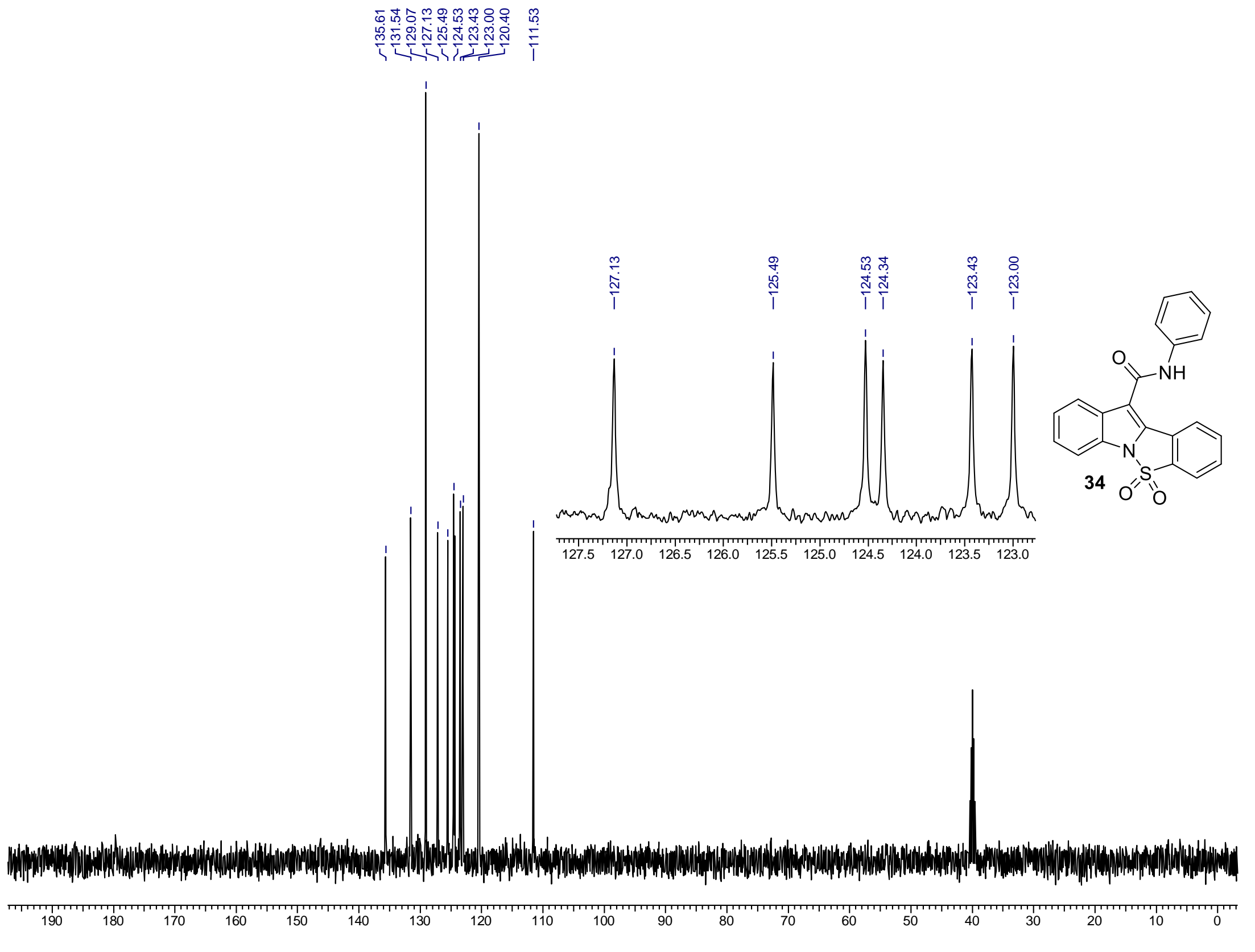


Chloroform-d

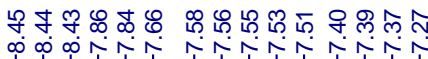

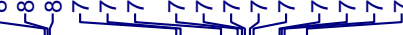
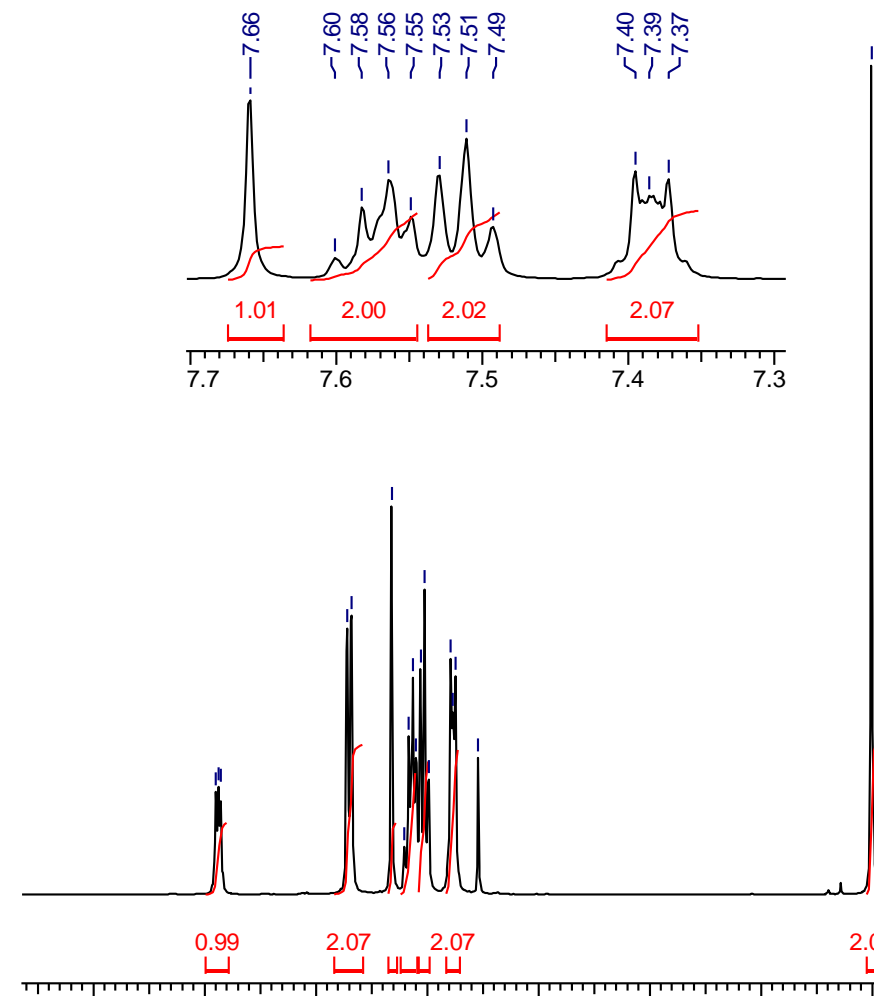
3.00

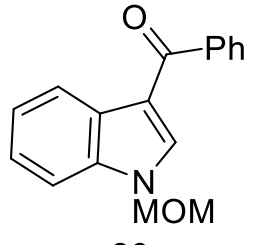

30 


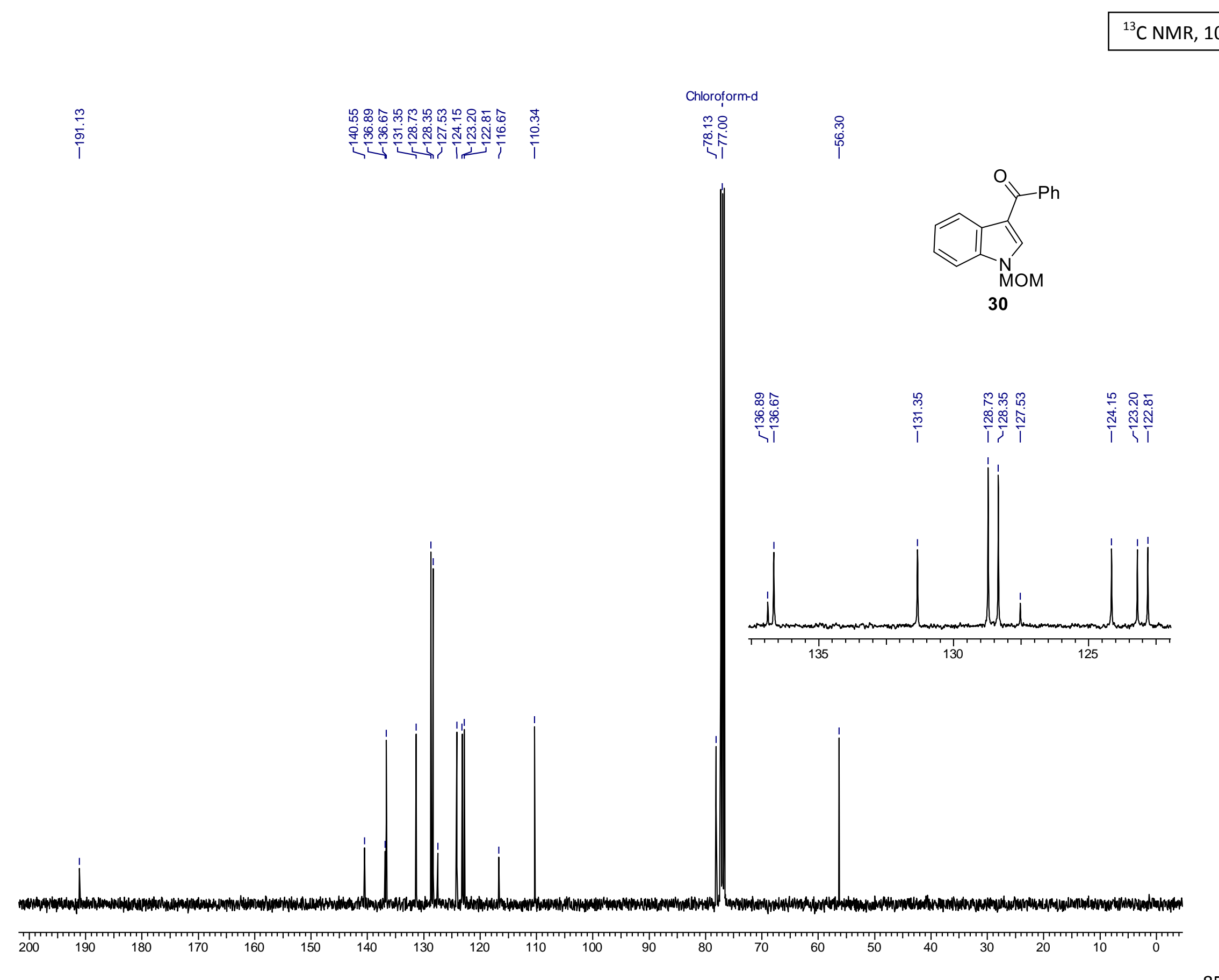




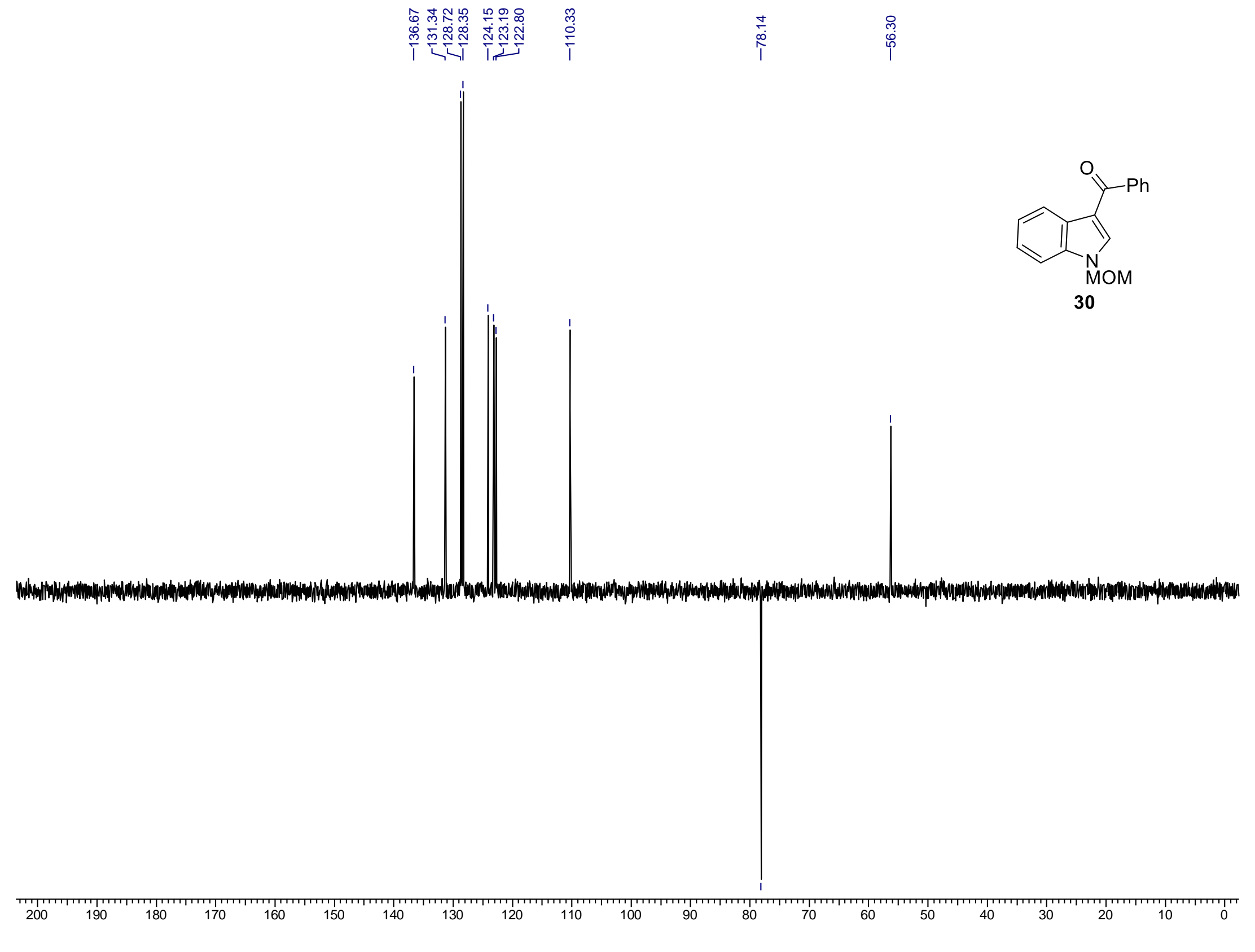




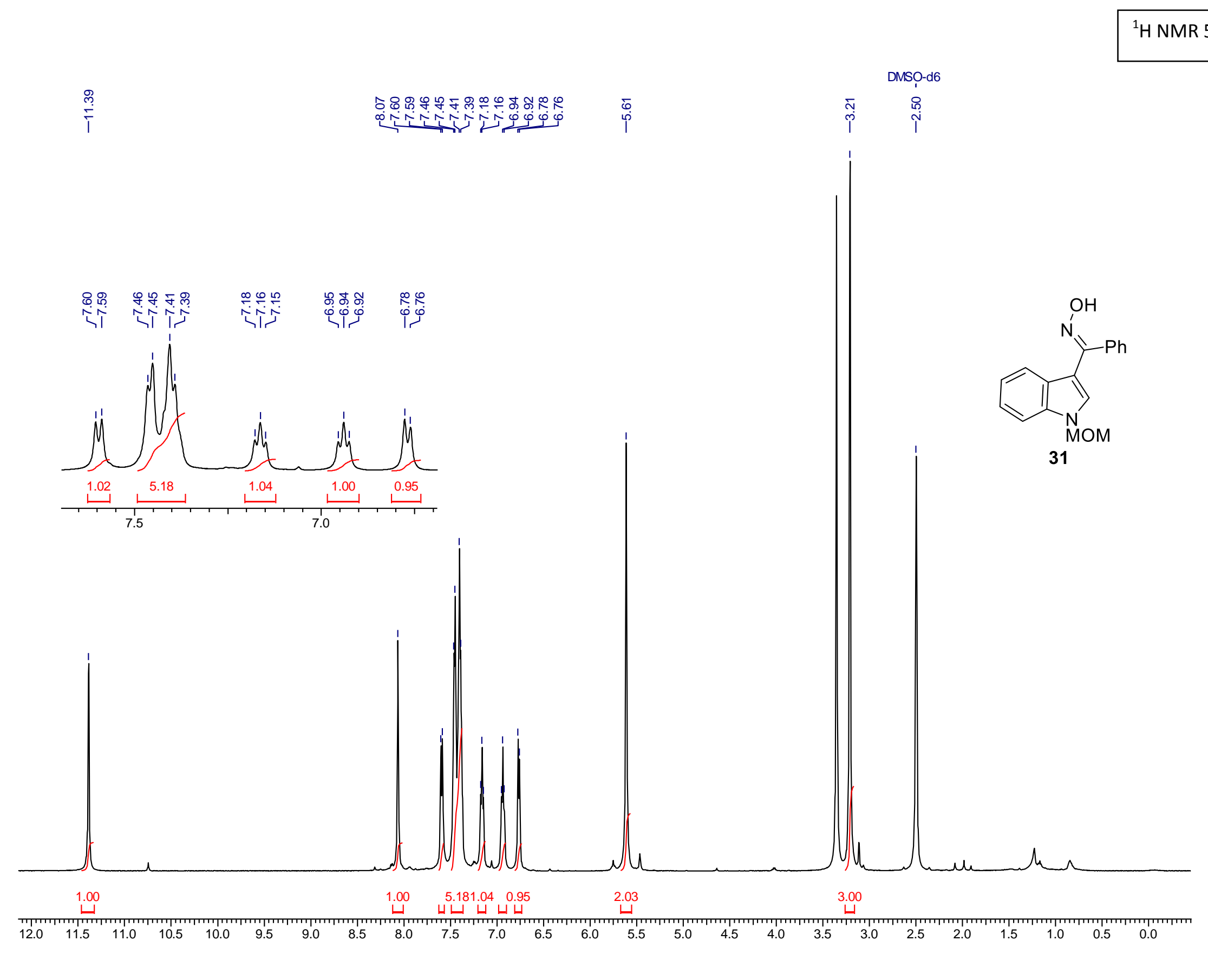


DMSO-d6

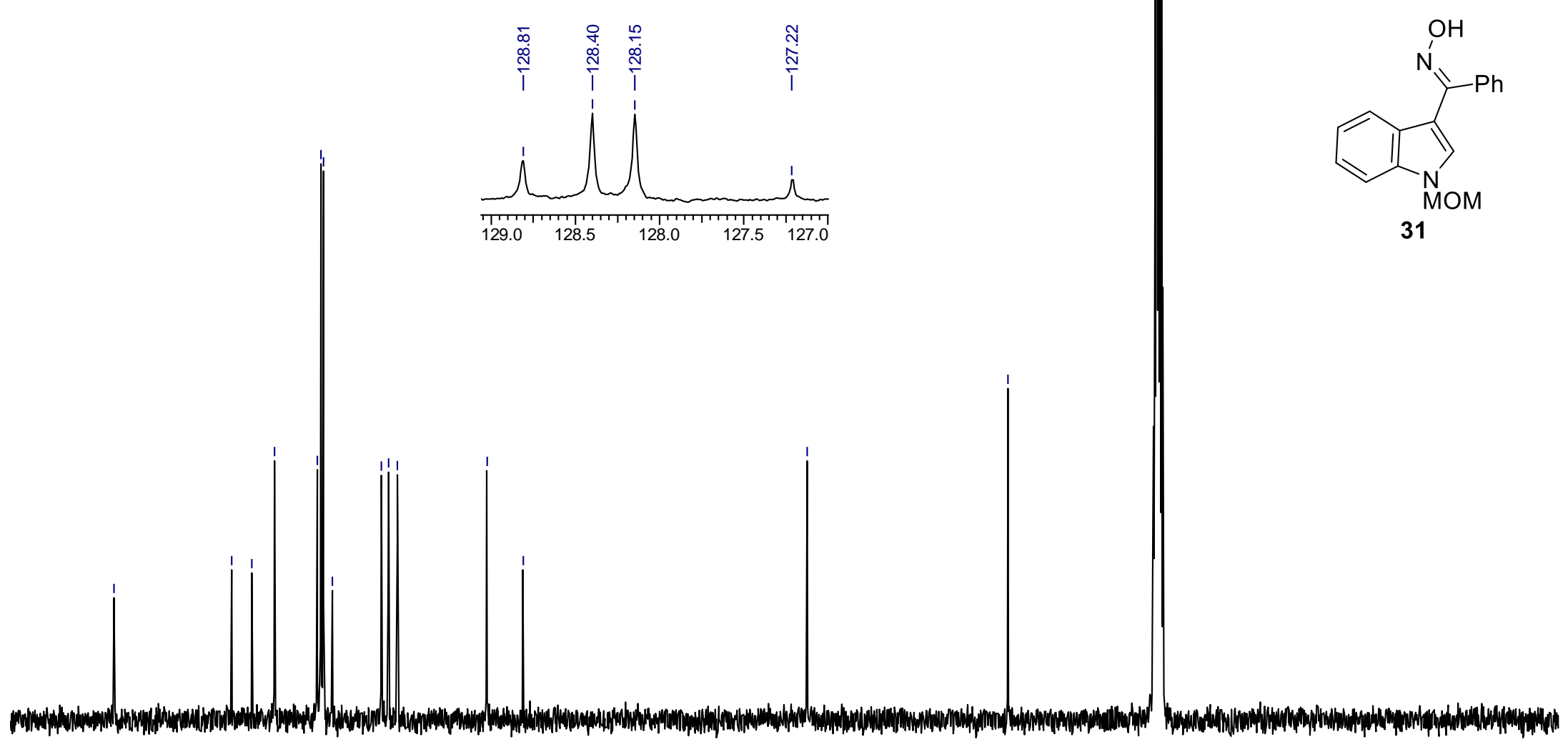




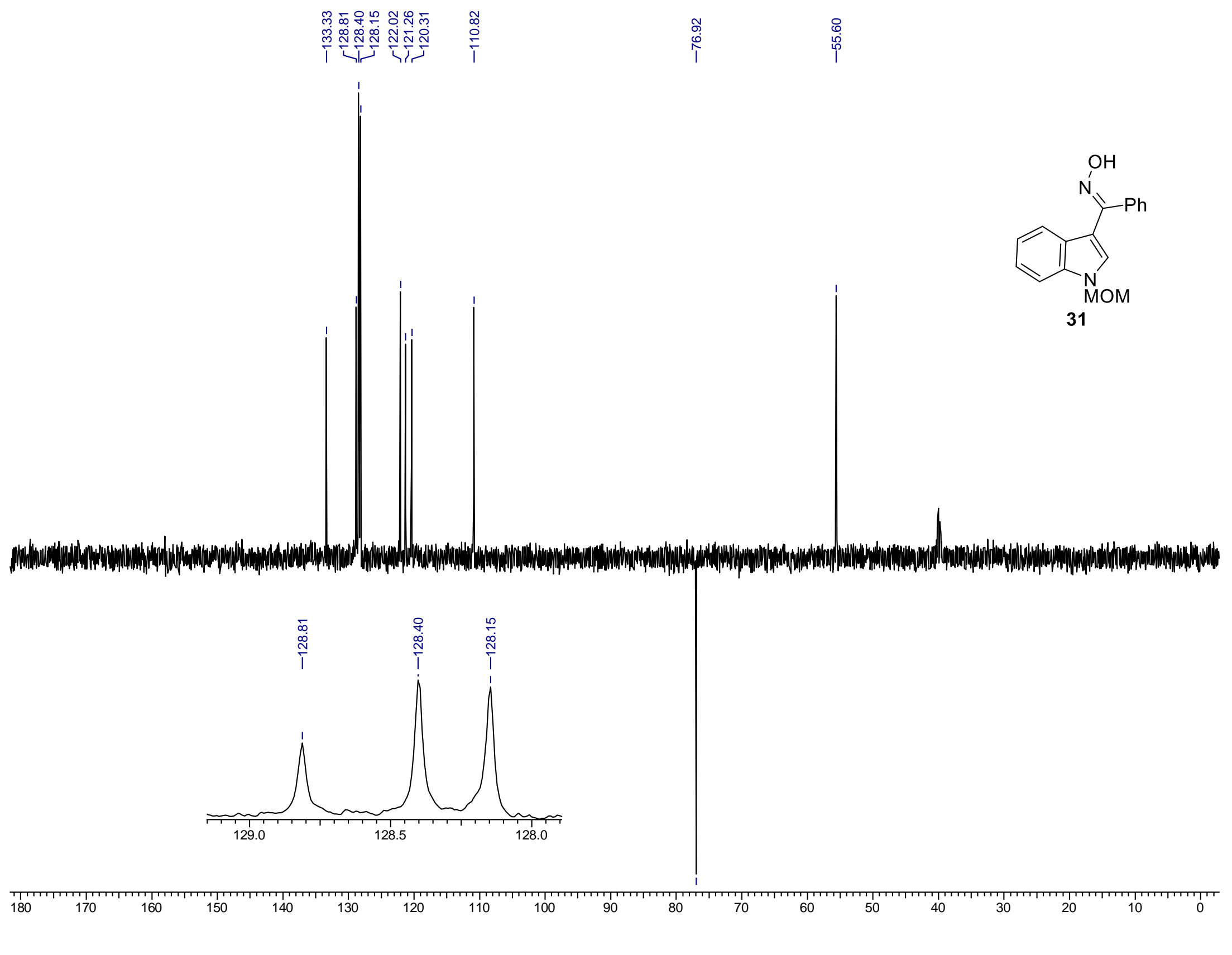


Chloroform-d

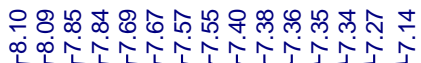

Chloroform-d

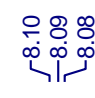

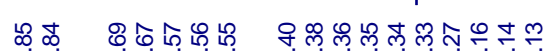
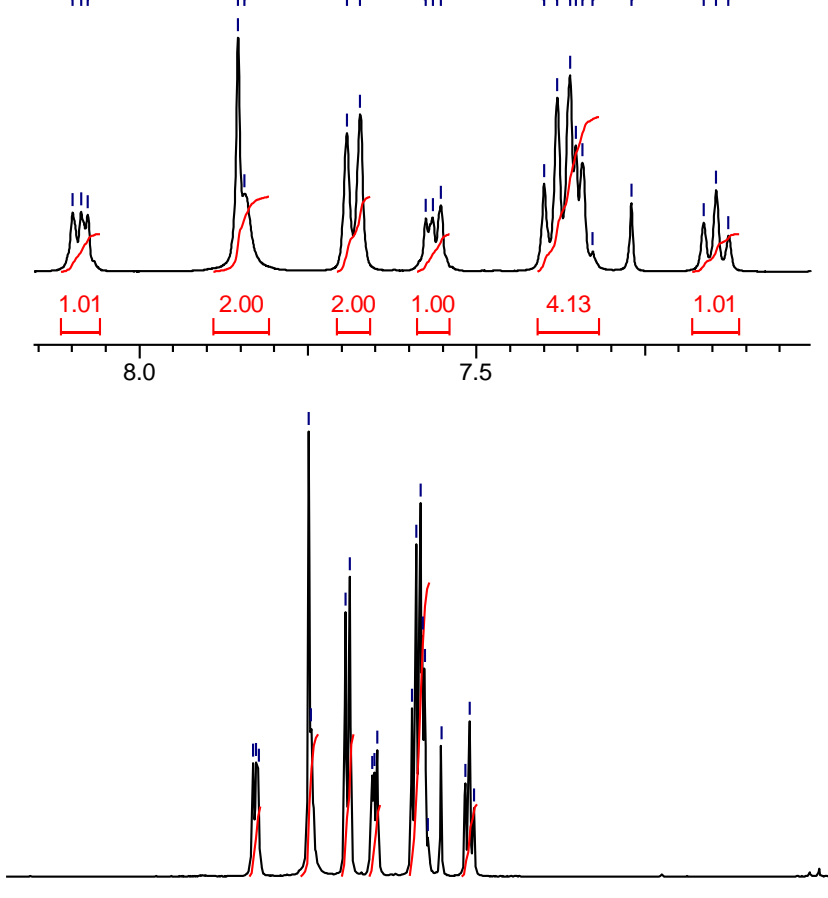

$1.012 .00 \quad 1.004 .131 .01$ 2.03 3.00

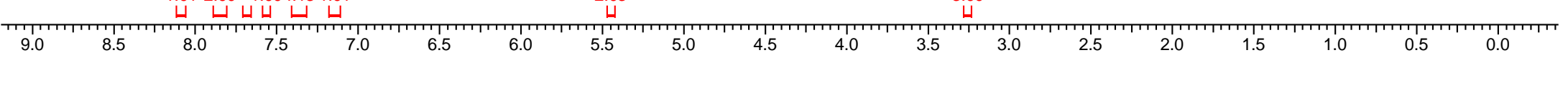




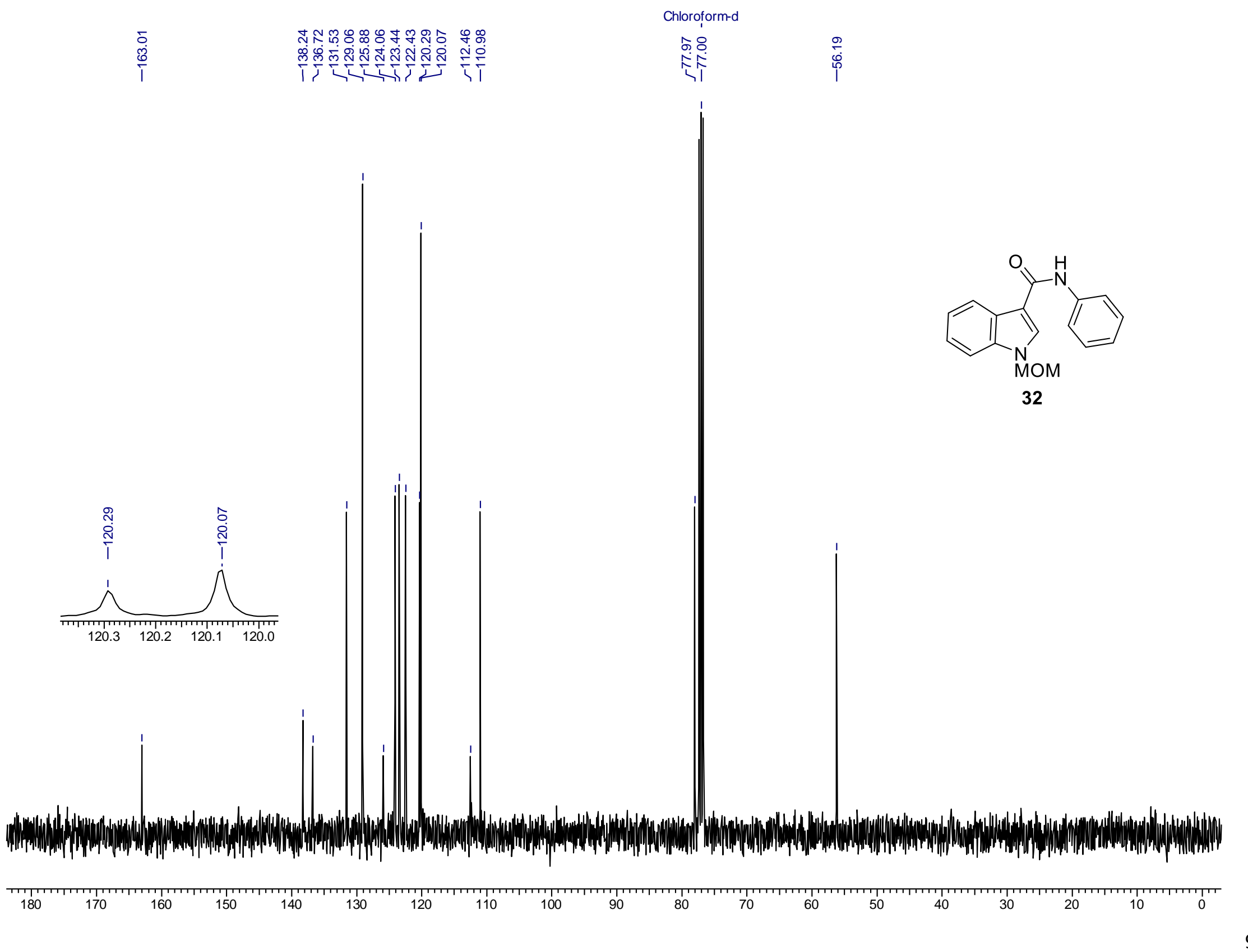




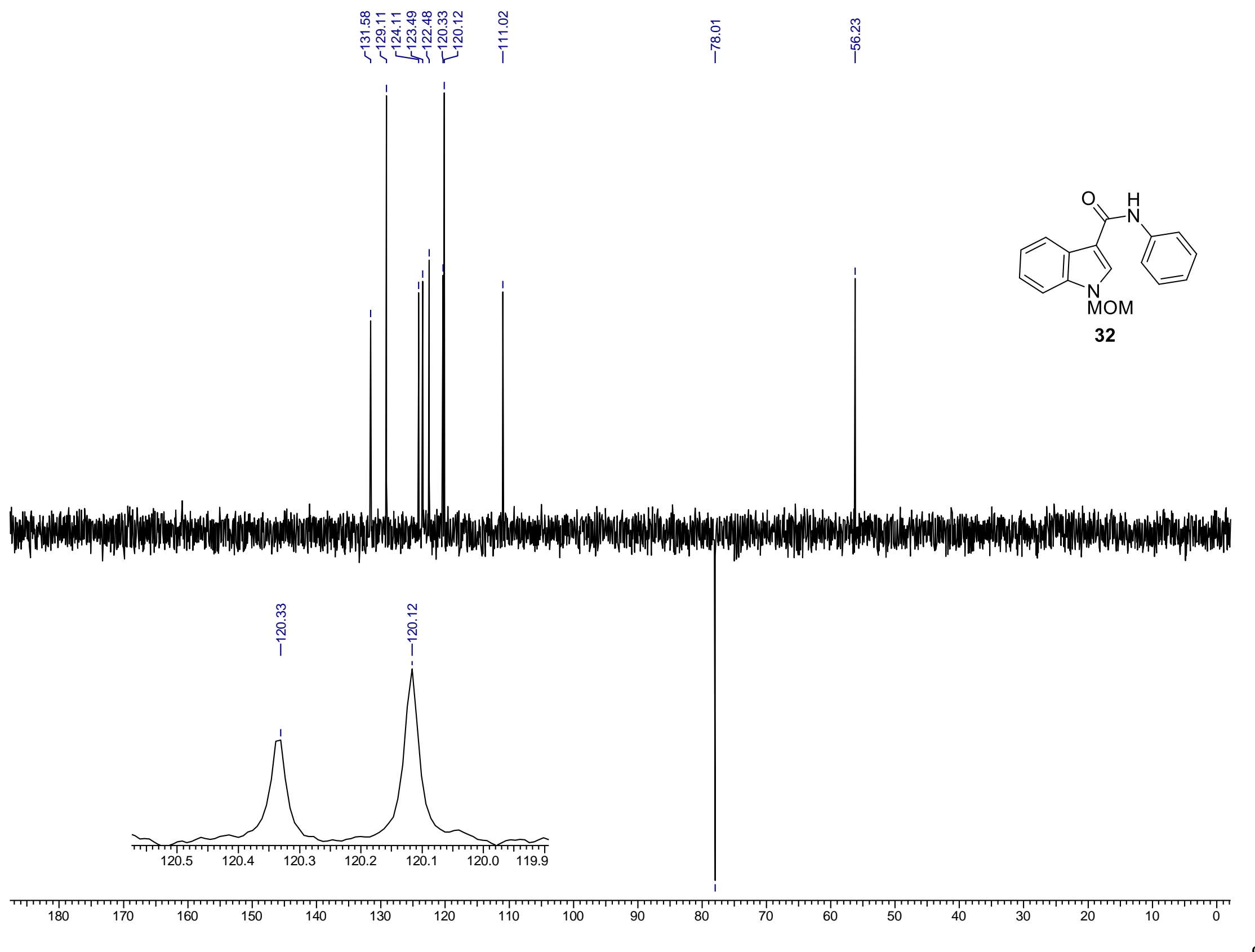



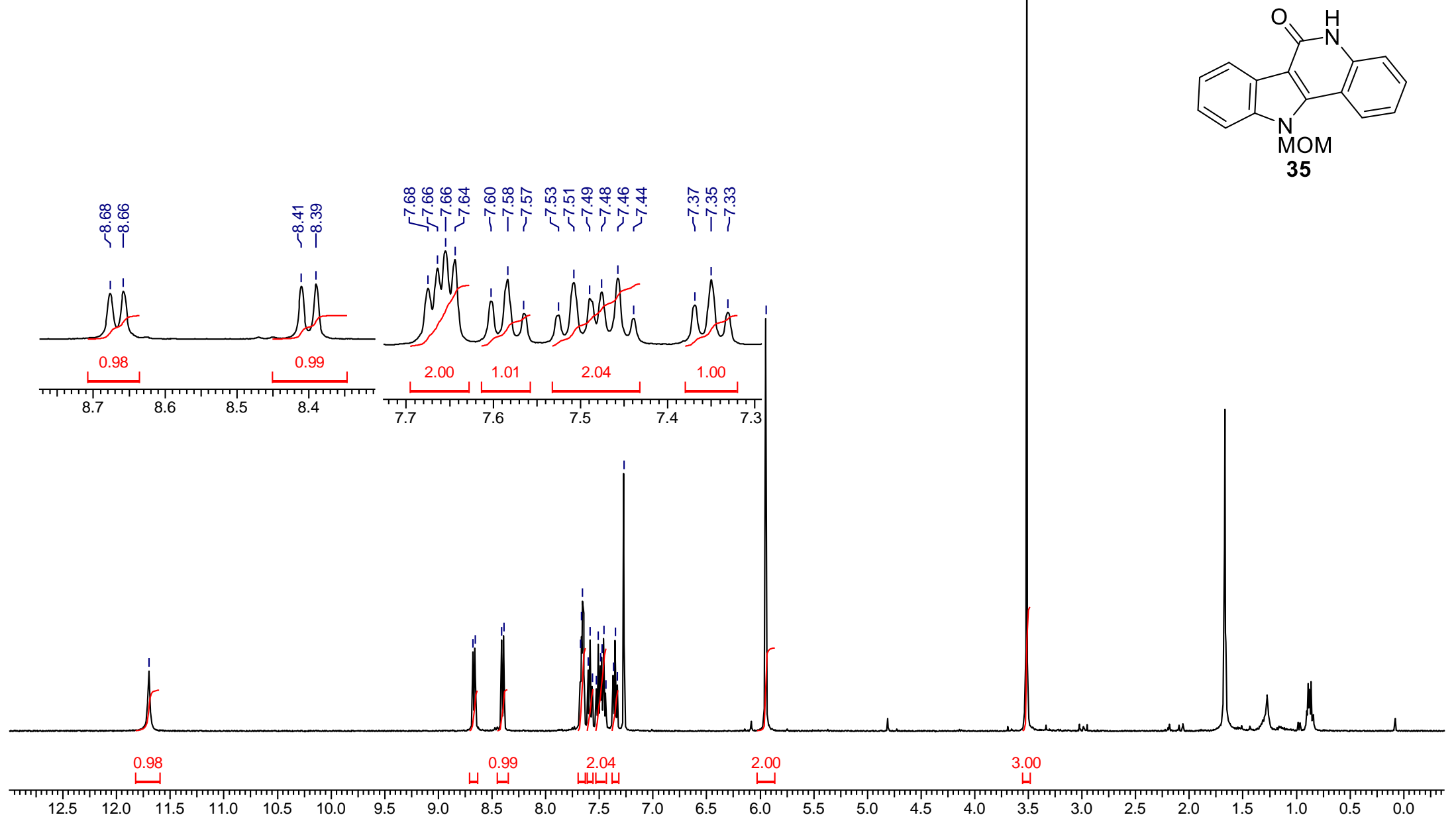
Chloroform-d
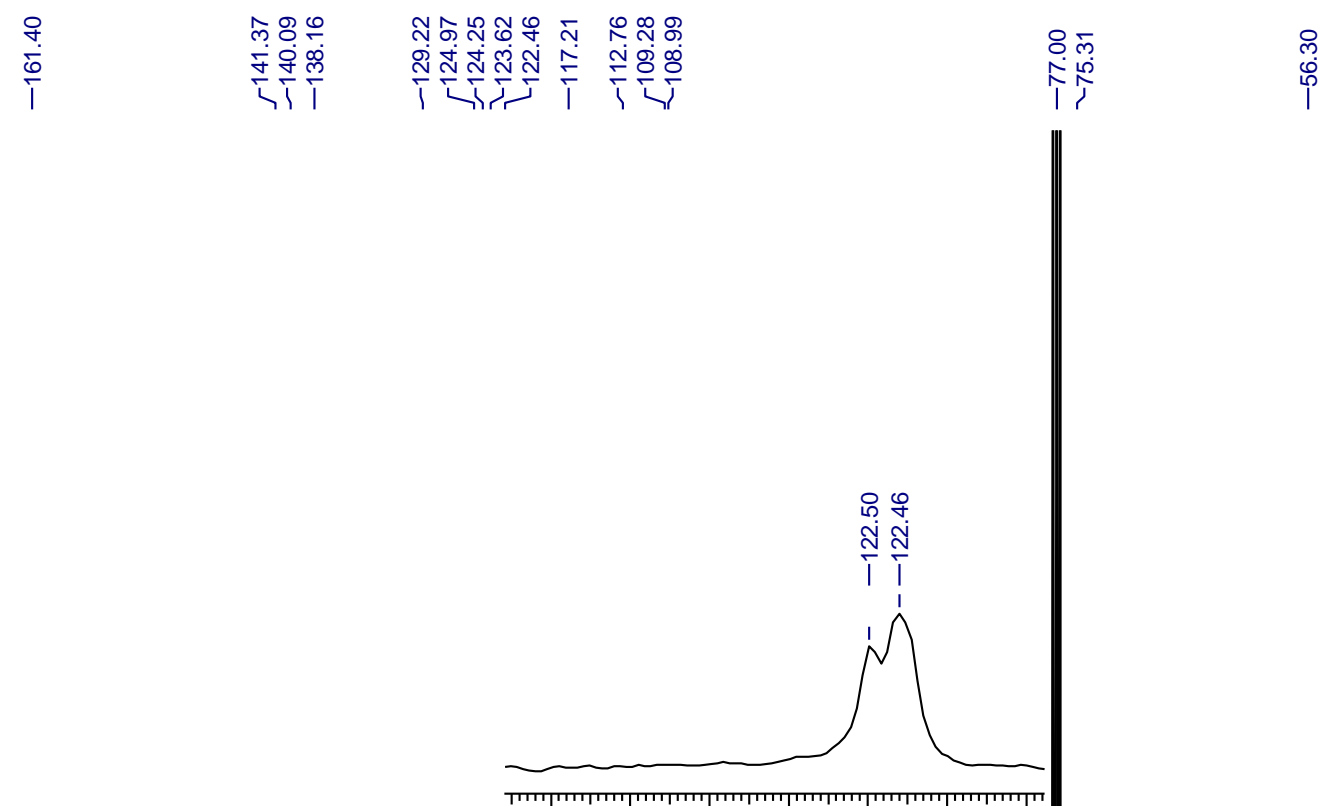

$\begin{array}{lllllll}122.9 & 122.8 & 122.7 & 122.6 & 122.5 & 122.4 & 122.3\end{array}$
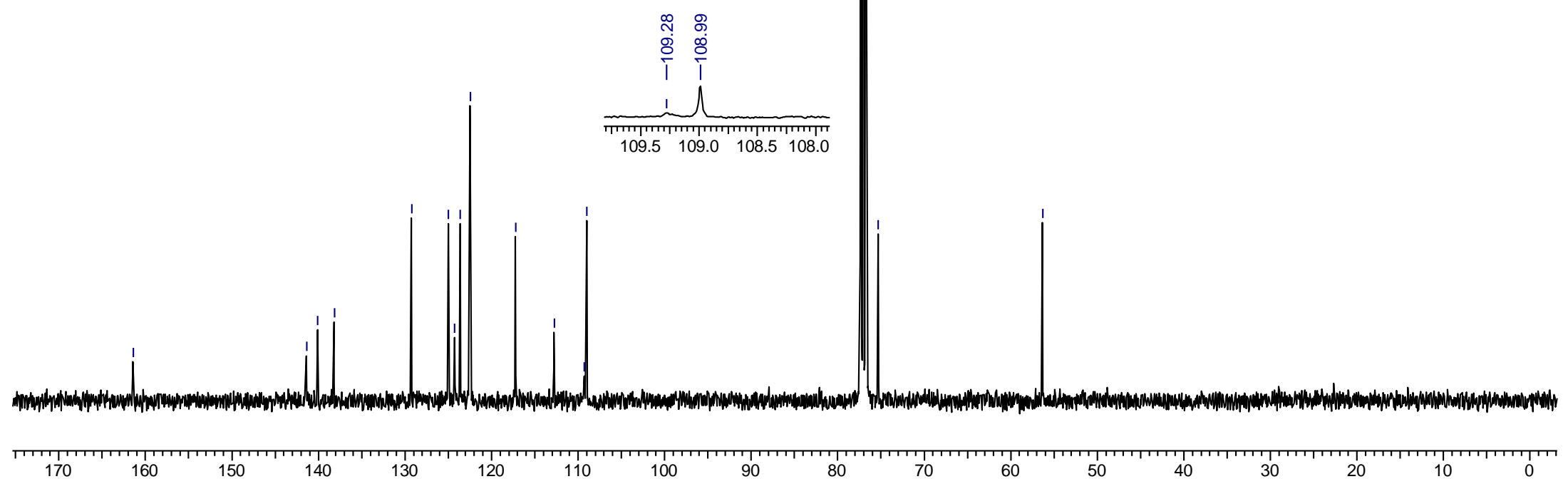

120

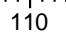

100

90

80

70

60

50

40

30

20 


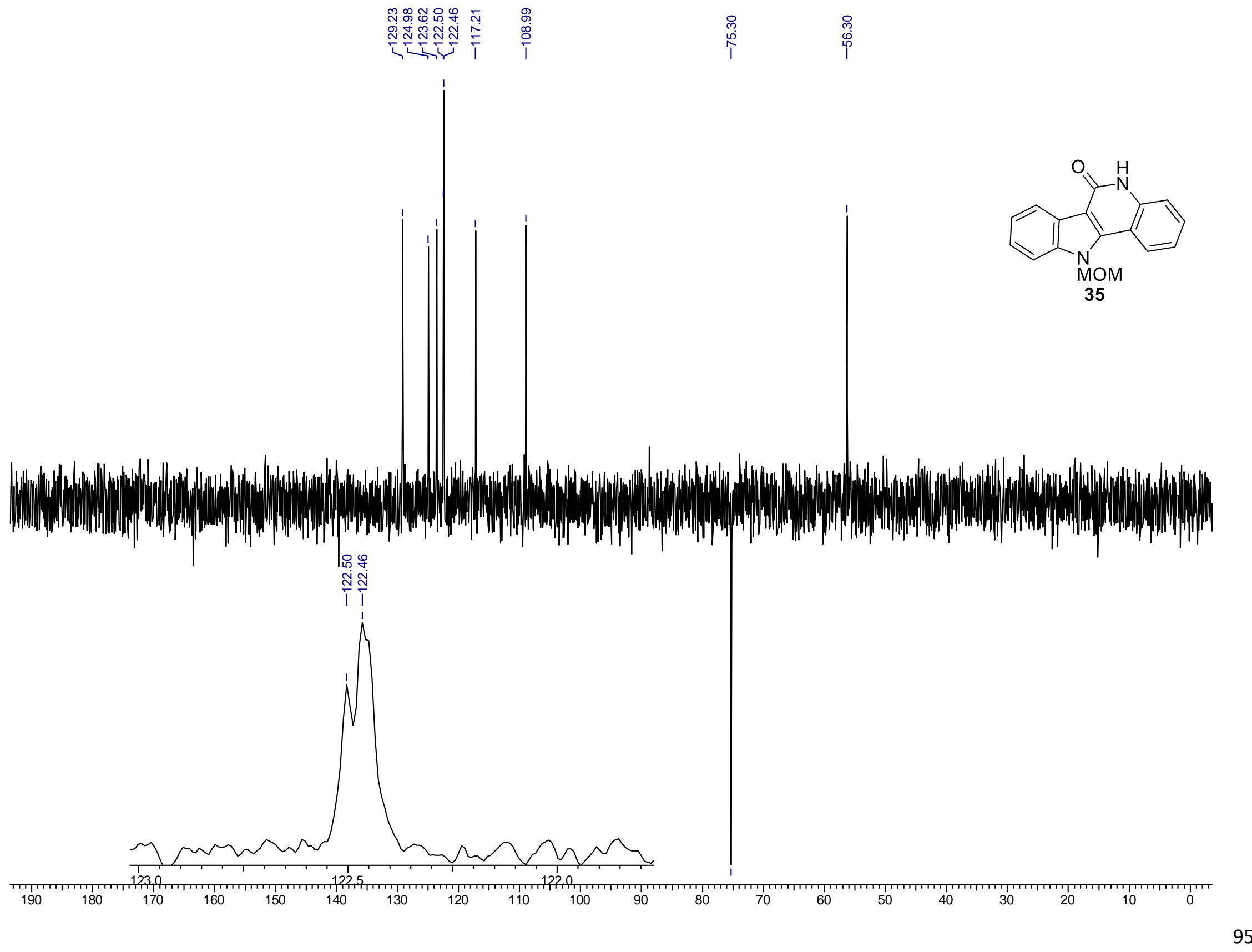




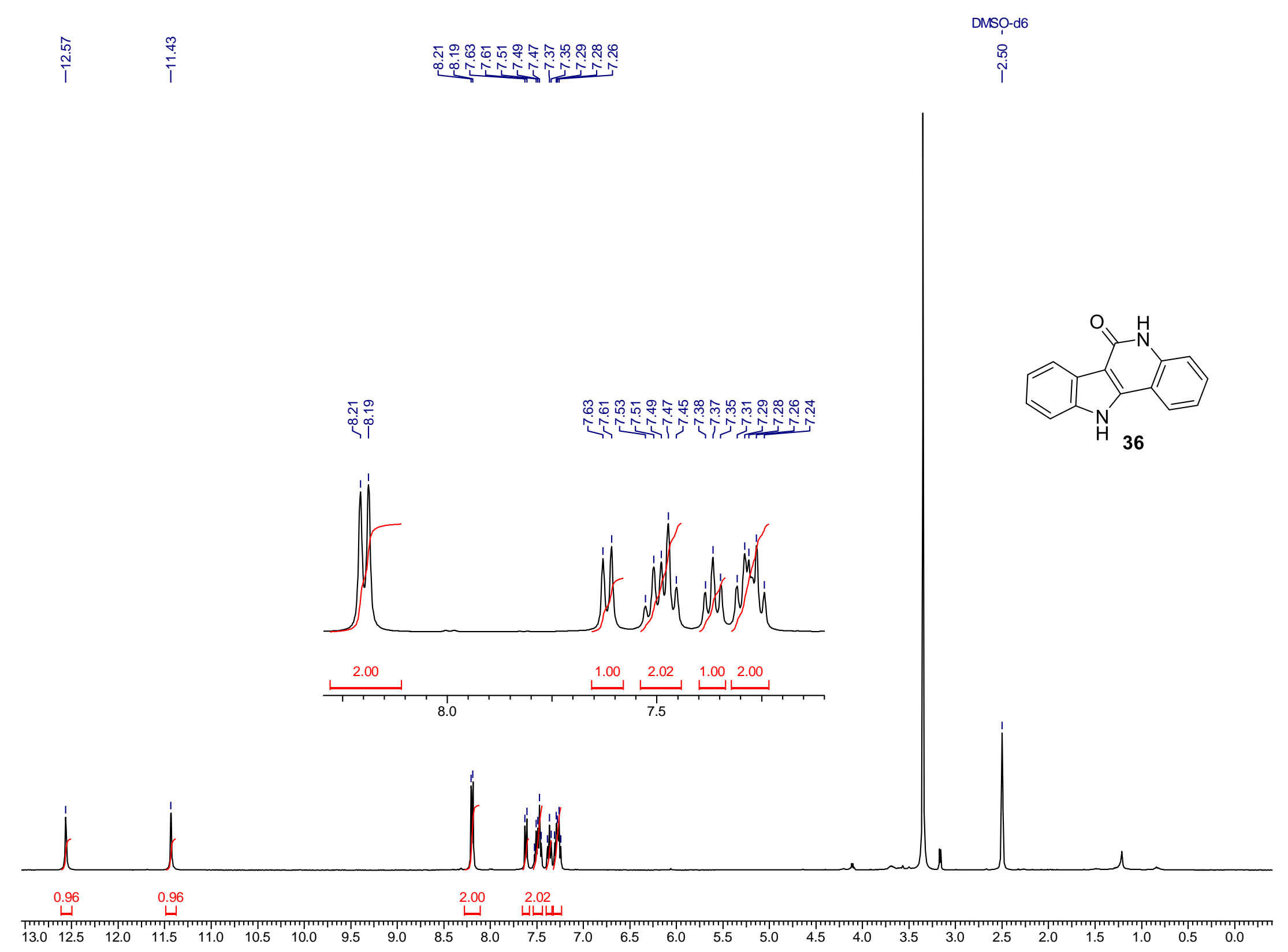




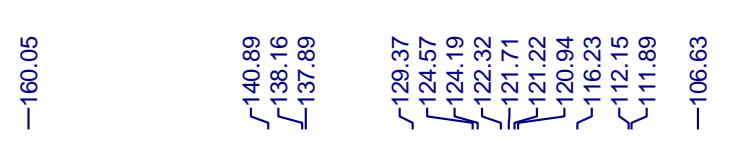

DMSO-d6

占尔
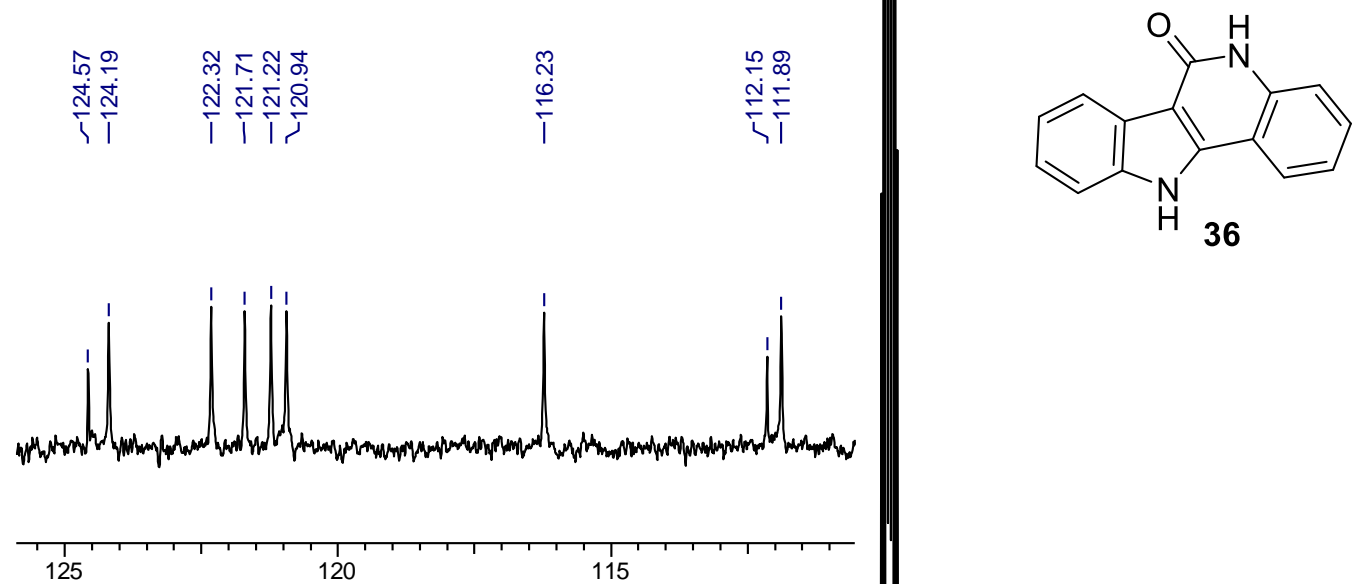

120

115

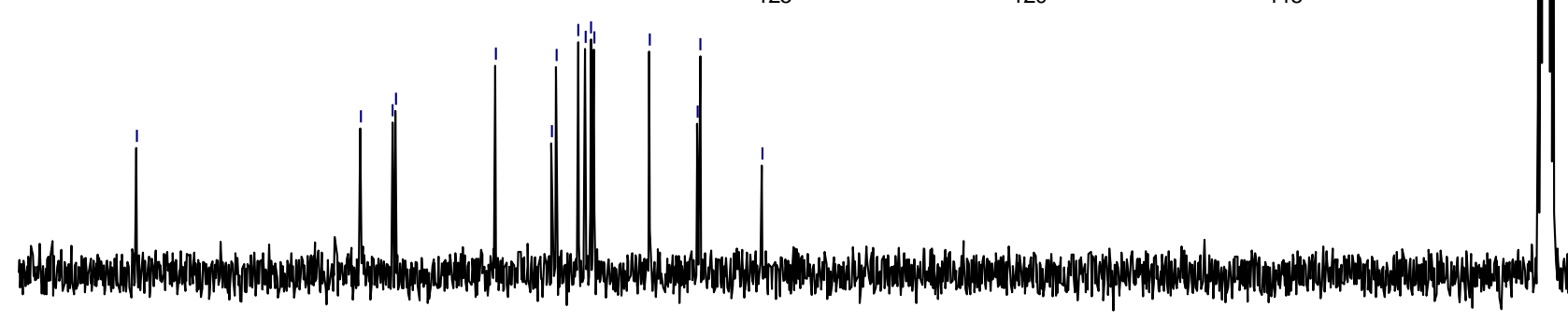

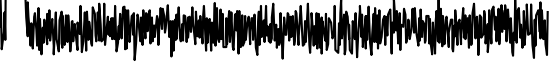

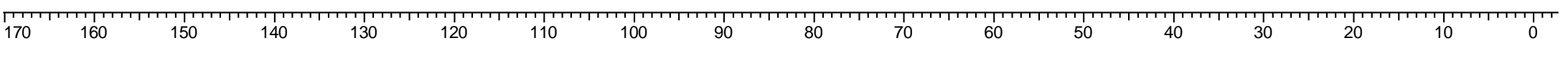




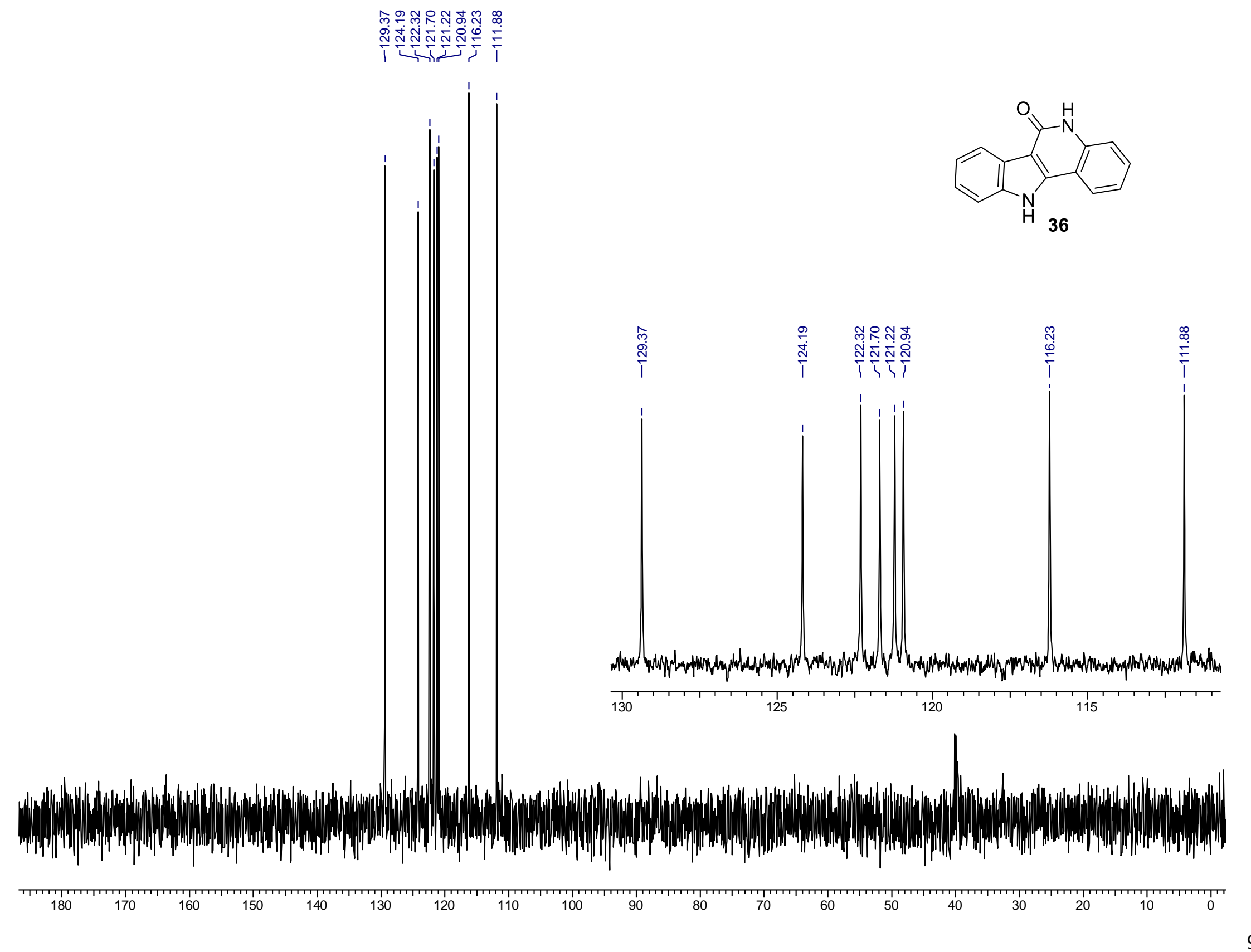




\section{Blank reaction to detect reactive intermediates:}
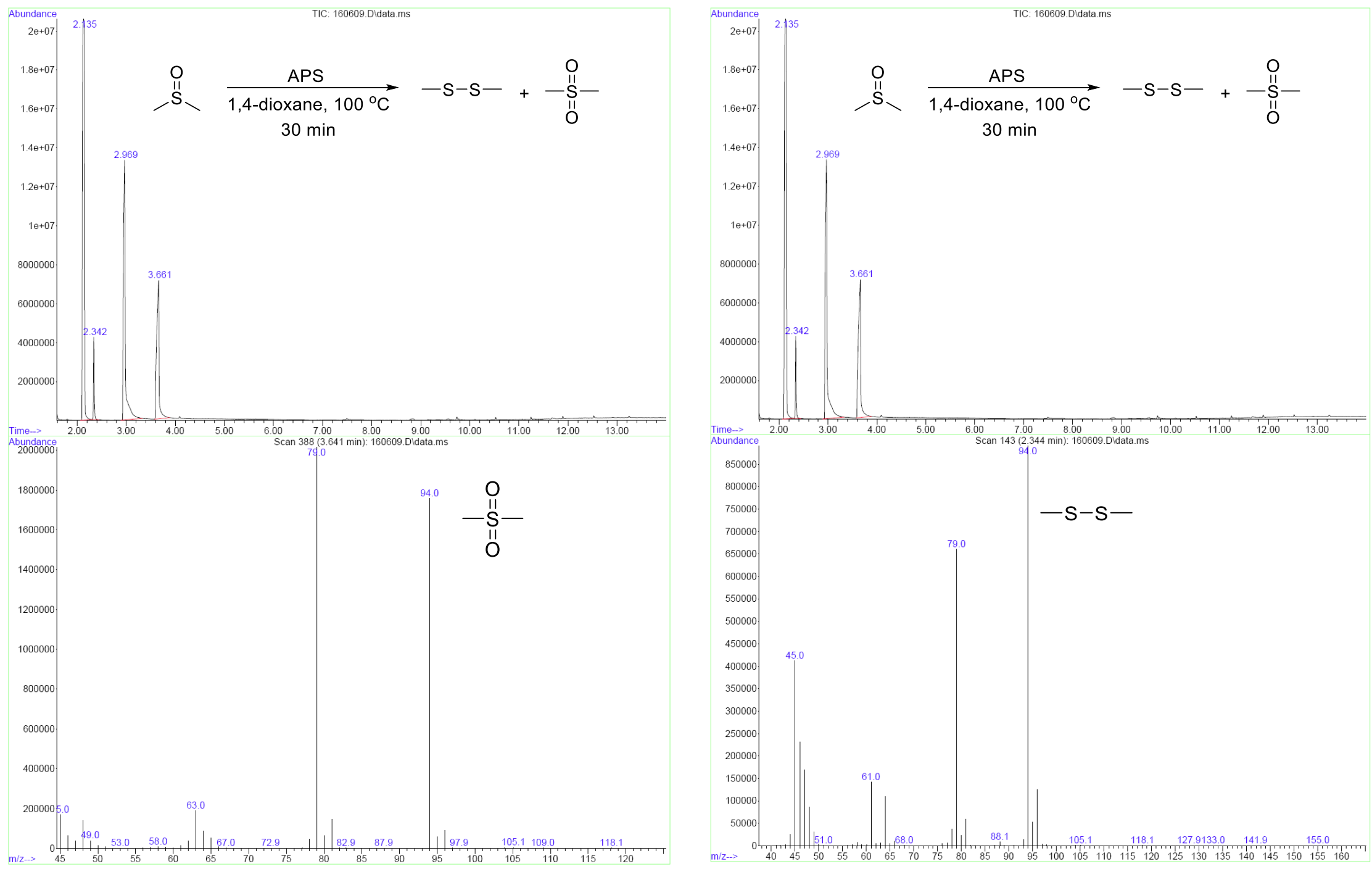
Trapping of intermediate 38a by ESI-LCMS:

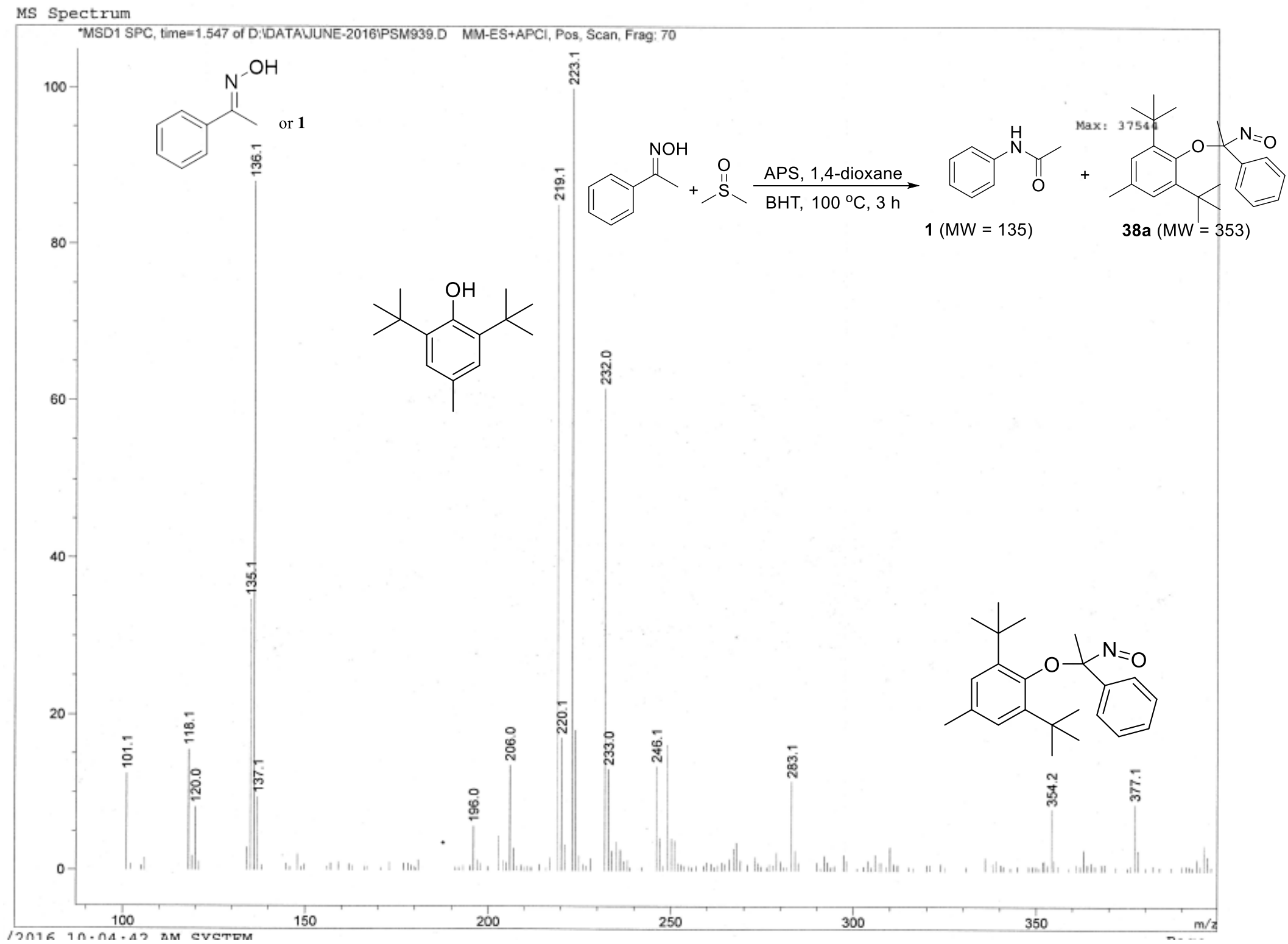




\section{Trapping of intermediate 38a by ESI-HRMS:}

PS-939 \#137 RT: $0.61 \quad$ AV: $1 \quad$ NL: $1.82 E 9$

T: FTMS + p ESI Full ms [100.00-1500.00]

$$
\begin{aligned}
& 136.0757 \\
& R=97307
\end{aligned}
$$

$\mathrm{C}_{8} \mathrm{H}_{10} \mathrm{ON}=136.0757$
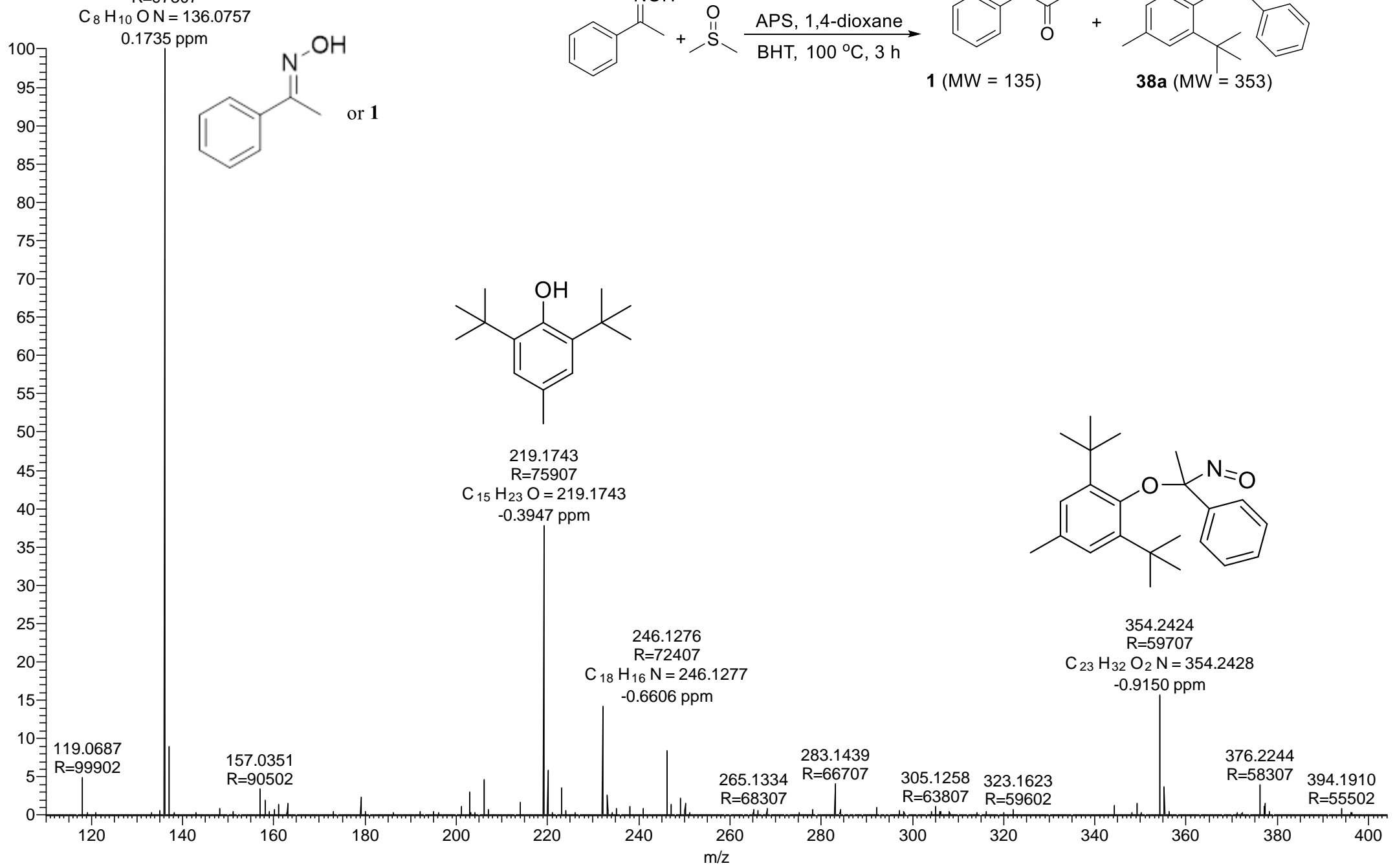
Trapping of intermediate $38 \mathrm{~b}$ by ESI-LCMS:

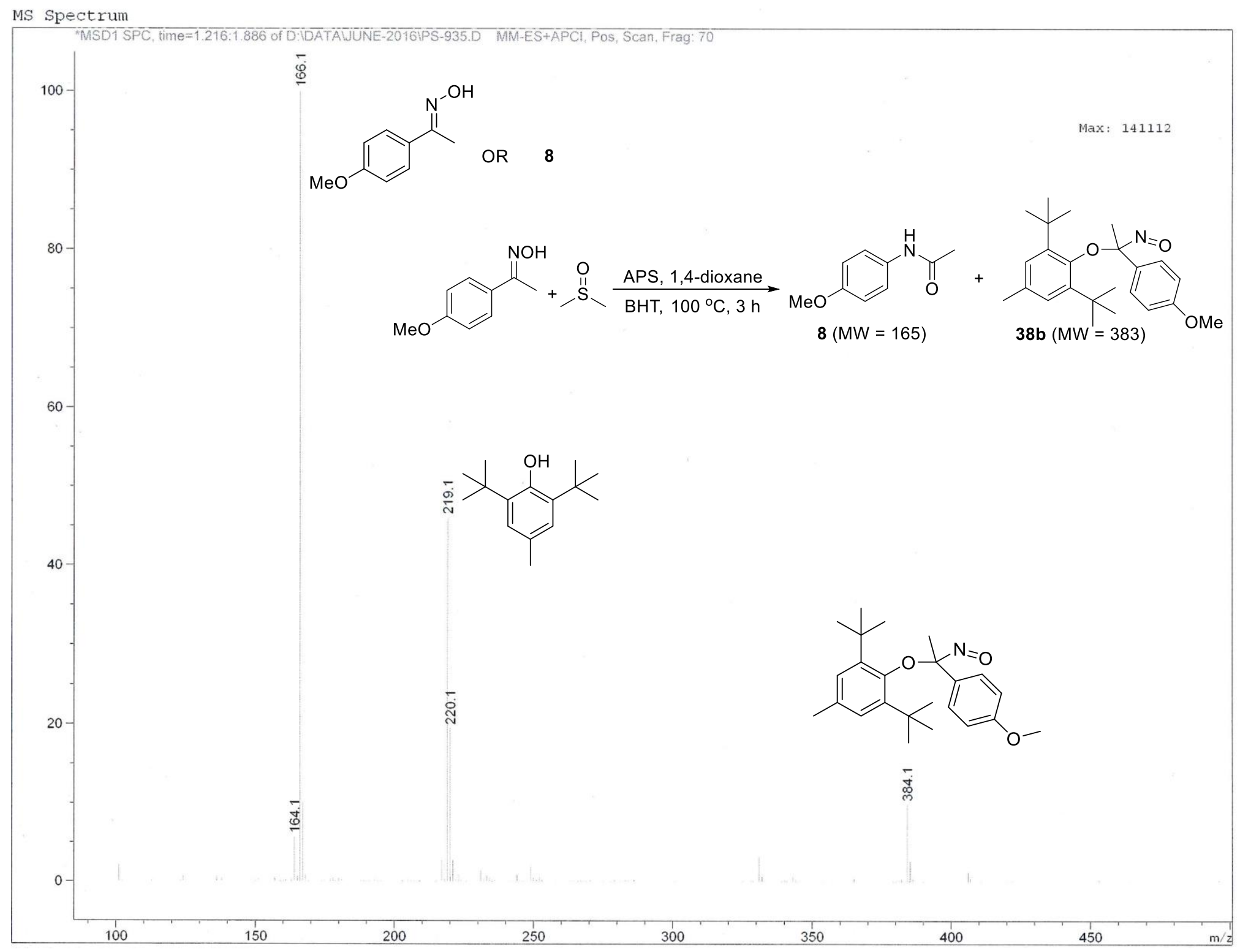




\section{Trapping of intermediate $38 \mathrm{~b}$ by ESI-LCMS:}

PS-935 \#127 RT: 0.56 AV: 1 NL: 6.50E9

T: FTMS + p ESI Full ms [100.00-1500.00]

166.0886

$\mathrm{C}_{9} \mathrm{H}_{12} \mathrm{O}_{2} \mathrm{~N}=166.0863$

100 - $14.0730 \mathrm{ppm}$

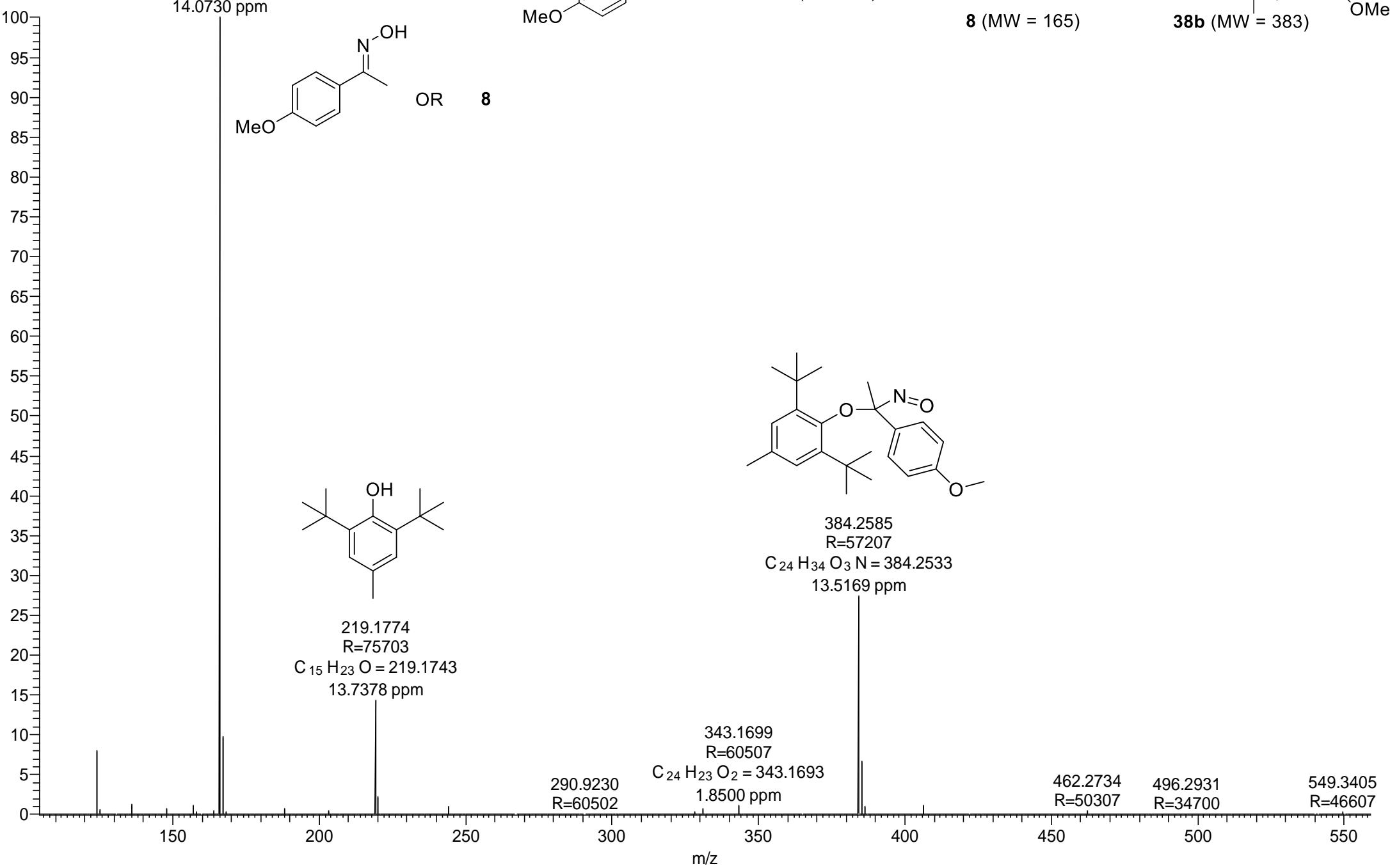




\section{Labeling Experiment:}

VTH-S1 \#108 RT: 0.48 AV: 1 NL: 2.22E9

T: FTMS + p ESI Full ms [100.00-1500.00]

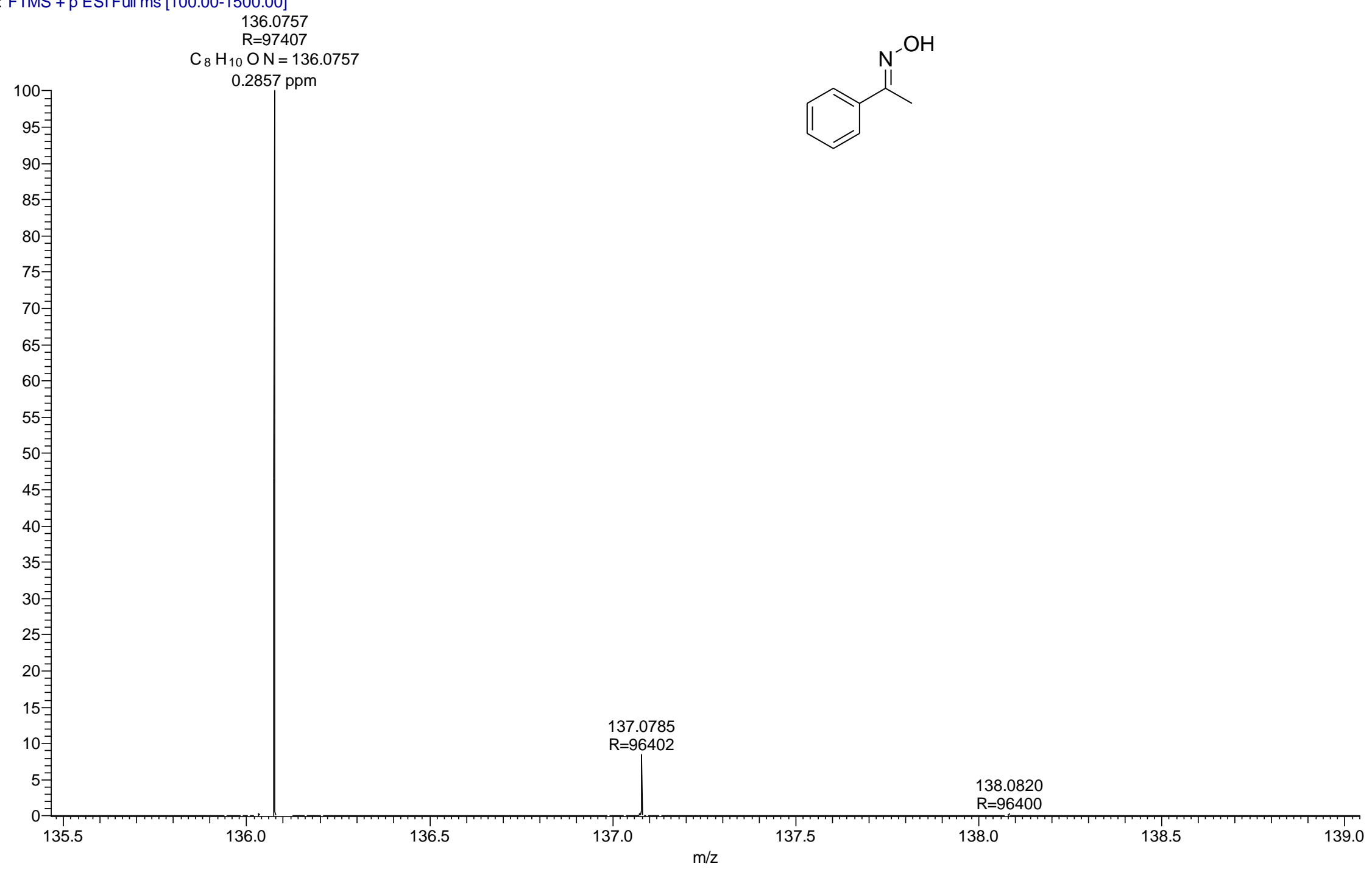


VTH-S2 \#107 RT: $0.47 \quad$ AV: 1 NL: 2.63E9

T: FTMS + p ESI Full ms [100.00-1500.00]

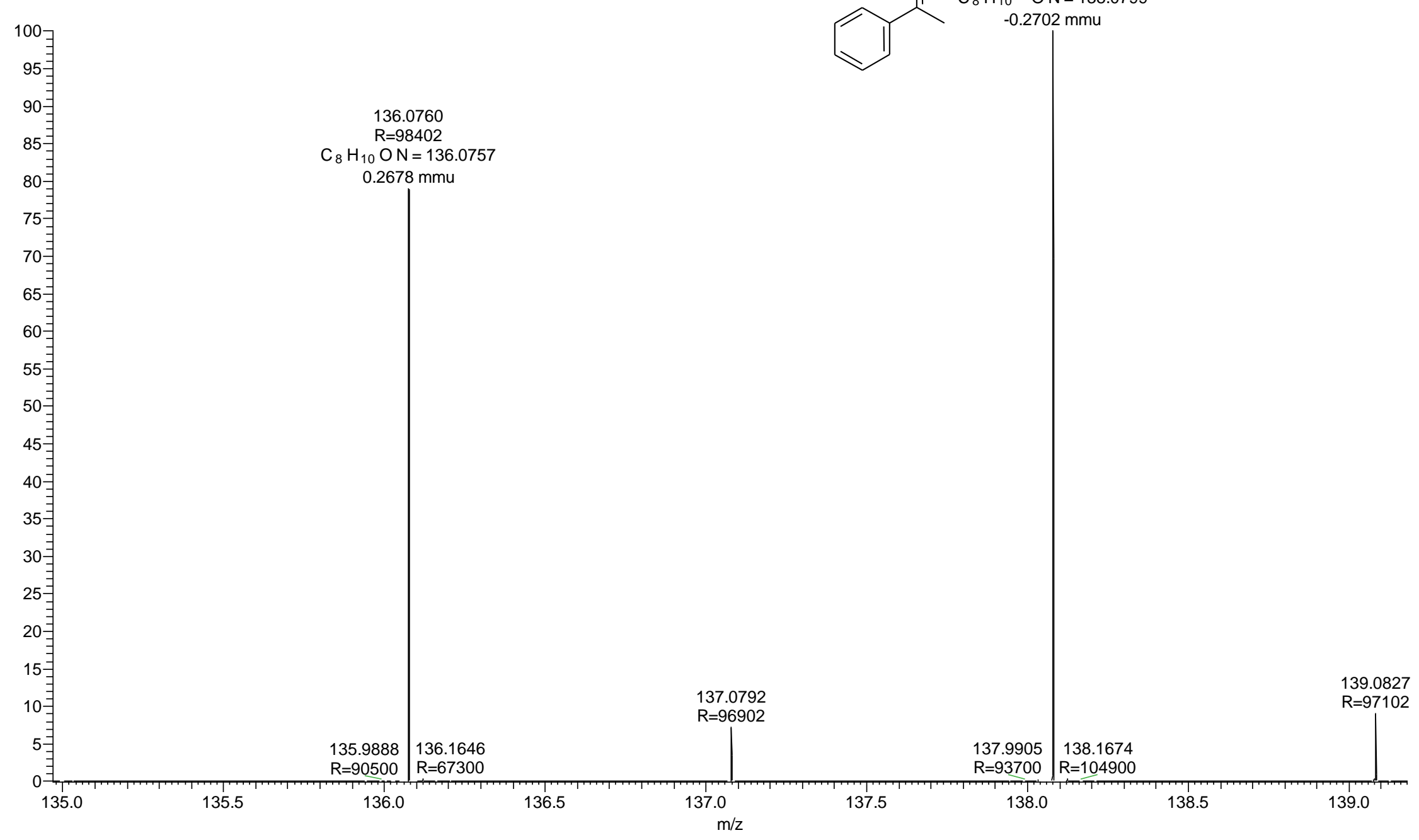


VTH-S3 \#102 RT: 0.45 AV: 1 NL: $8.91 \mathrm{E} 8$

T: FTMS + p ESI Full ms [100.00-1500.00]

136.0758

$\mathrm{C}_{8} \mathrm{H}_{10} \mathrm{ON}=136.0757$

$0.0694 \mathrm{mmu}$
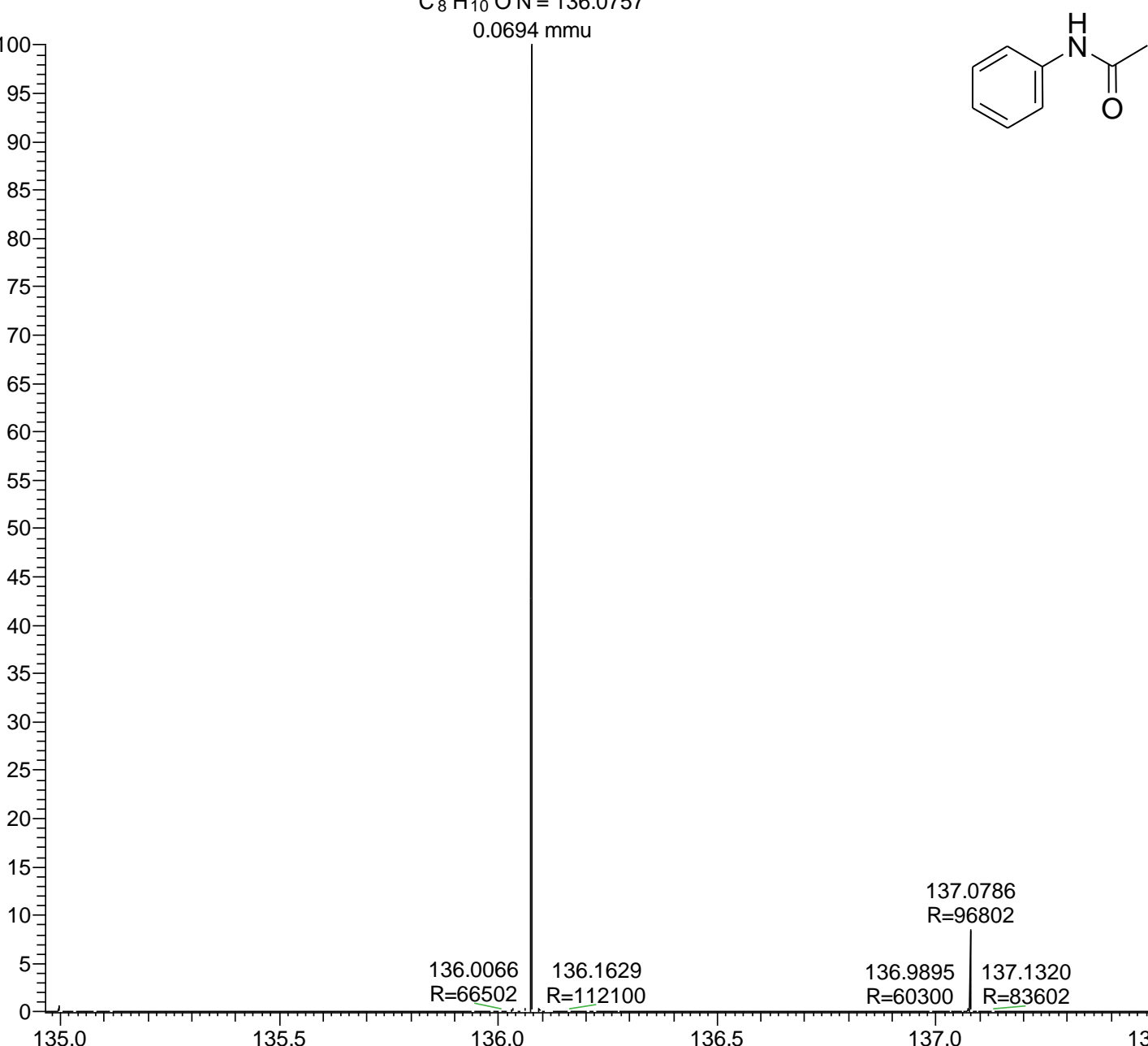

136.0

$R=112100$

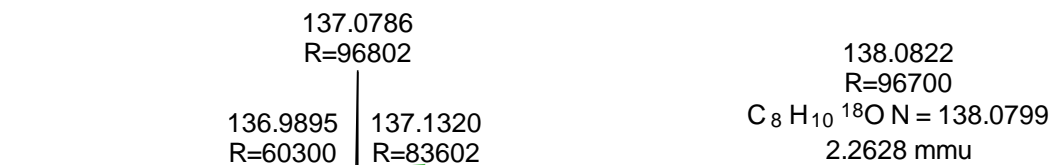

$\mathrm{R}=60300 \mathrm{R}=83602$

137.5

$2.2628 \mathrm{~mm}$ 
VTH-S4 \#104 RT: 0.46 AV: 1 NL: 4.59E8

T: FTMS + p ESI Full ms [100.00-1500.00]

36.0757

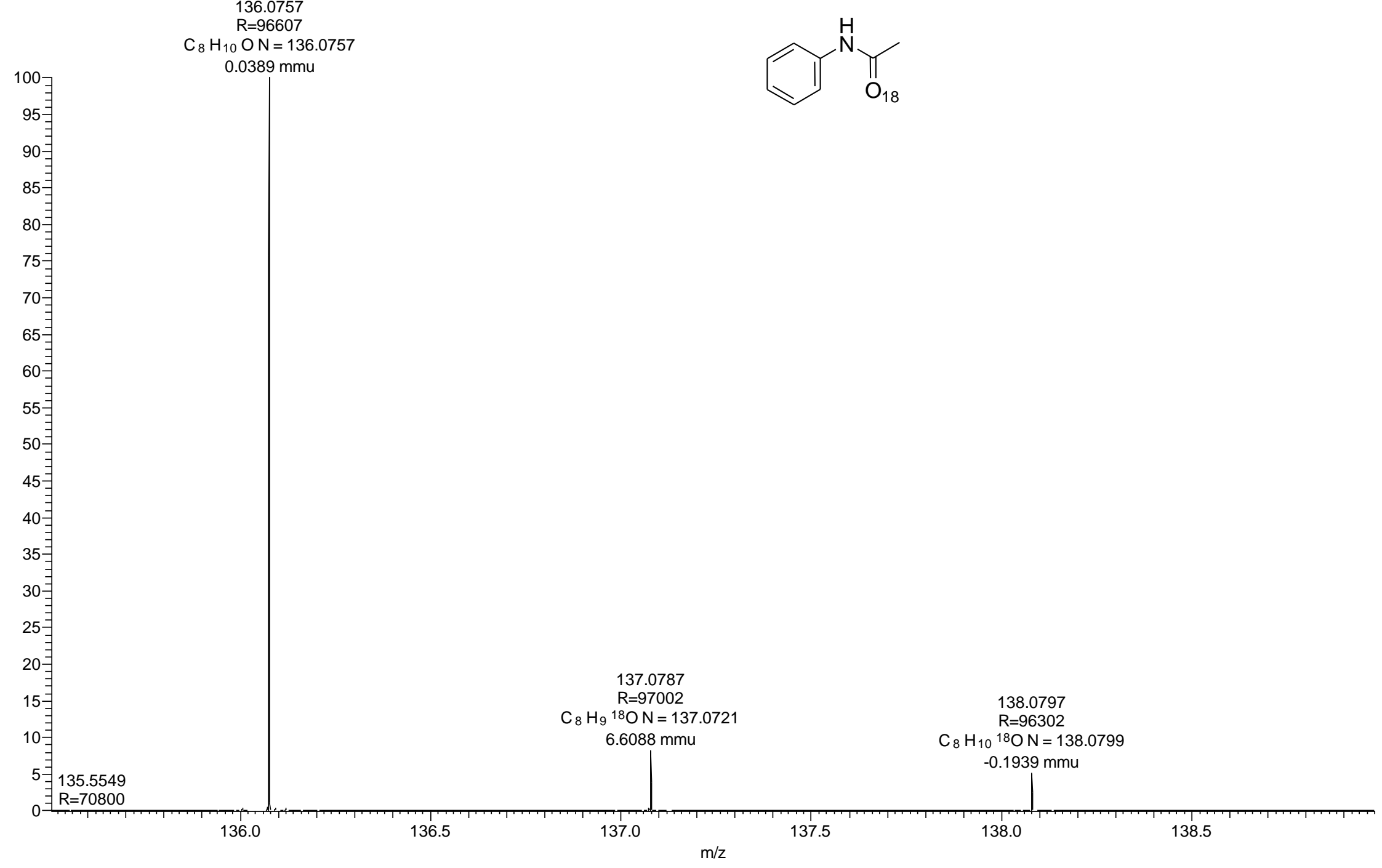

